\title{
LOCALLY NORMAL SUBGROUPS OF TOTALLY DISCONNECTED GROUPS. PART II: COMPACTLY GENERATED SIMPLE GROUPS
}

\author{
PIERRE-EMMANUEL CAPRACE ${ }^{1}$, COLIN D. REID $^{2}$ and \\ GEORGE A. WILLIS ${ }^{2}$ \\ ${ }^{1}$ Université catholique de Louvain, IRMP, Chemin du Cyclotron 2, bte L7.01.02, \\ 1348 Louvain-la-Neuve, Belgique; \\ email: pe.caprace@uclouvain.be \\ ${ }^{2}$ University of Newcastle, School of Mathematical and Physical Sciences, Callaghan, \\ NSW 2308, Australia; \\ email: Colin.Reid@newcastle.edu.au, George.Willis@newcastle.edu.au
}

Received 25 November 2014; accepted 15 March 2017

\begin{abstract}
We use the structure lattice, introduced in Part I, to undertake a systematic study of the class $\mathscr{S}$ consisting of compactly generated, topologically simple, totally disconnected locally compact groups that are nondiscrete. Given $G \in \mathscr{S}$, we show that compact open subgroups of $G$ involve finitely many isomorphism types of composition factors, and do not have any soluble normal subgroup other than the trivial one. By results of Part I, this implies that the centralizer lattice and local decomposition lattice of $G$ are Boolean algebras. We show that the $G$-action on the Stone space of those Boolean algebras is minimal, strongly proximal, and microsupported. Building upon those results, we obtain partial answers to the following key problems: Are all groups in $\mathscr{S}$ abstractly simple? Can a group in $\mathscr{S}$ be amenable? Can a group in $\mathscr{S}$ be such that the contraction groups of all of its elements are trivial?
\end{abstract}

2010 Mathematics Subject Classification: 22D05 (primary); 20E15, 20E32 (secondary)

\section{Introduction}

1.1. Background. The solution to Hilbert's fifth problem [43, Theorem 4.6] ensures that a connected locally compact group is in fact an inverse limit of Lie groups. In particular, the general structure theory of connected locally compact groups largely reduces to that of simple Lie groups, soluble Lie groups, and

(c) The Author(s) 2017. This is an Open Access article, distributed under the terms of the Creative Commons Attribution licence (http://creativecommons.org/licenses/by/4.0/), which permits unrestricted re-use, distribution, and reproduction in any medium, provided the original work is properly cited. 
compact groups (which are themselves inverse limits of compact Lie groups), and the structure of connected simple locally compact groups is thoroughly understood.

The possibility of a structure theory of locally compact groups beyond the connected case has become apparent over the last few decades. At least from a local perspective, one has an immediate reduction to the case of groups that are compactly generated, that is that admit a compact generating set: any locally compact group $G$ is a directed union of compactly generated open subgroups. In particular, every connected locally compact group is compactly generated. There is also a developing structure theory of closed normal subgroups of locally compact groups, for which the base case is groups that are topologically simple, that is, groups whose only closed normal subgroups are the identity subgroup and the whole group. In contrast to topological simplicity, we say a topological group is abstractly simple if it has no proper nontrivial normal subgroup (including dense normal subgroups).

Results in [18] and their recent extensions in [50] suggest that, under mild assumptions that exclude discrete groups, compactly generated topologically simple locally compact groups play a critical role in the structure of general locally compact groups, generalizing the status of simple Lie groups in the structure theory of connected locally compact groups. (A complementary role, analogous to the role of soluble Lie groups in Lie theory, could be played by the class of elementary groups introduced by Wesolek [67].) We shall exclude discrete simple groups from consideration, since their known behaviour precludes any structure theory having general scope. Indeed, the impossibility of such a theory can be given a precise mathematical formulation; see [60].

We thus arrive at the class $\mathscr{S}$ of nondiscrete, compactly generated, topologically simple, totally disconnected, locally compact groups, which is the focus of the present article. For the sake of brevity, we shall write t.d.l.c. for totally disconnected locally compact. Many specific families of examples of groups in $\mathscr{S}$ are known; see Appendix A. Our goal is to understand the general properties of the groups in $\mathscr{S}$. Some of the results presented here have been announced in [19]; the proofs rely on general tools developed in [21]. To make this discussion precise, let us make a list of questions about groups in $\mathscr{S}$. The answer to all of the following questions is known to be 'no' for every known example of a group in $\mathscr{S}$, which gives some reason to believe the answer should be 'no' in general.

For all the questions below, let $G \in \mathscr{S}$.

\section{QUESTION 1. Can G have a proper dense normal subgroup?}


QUESTION 2. Can $G$ have an infinite nonopen commensurated compact subgroup?

Question 3. Can $G$ be amenable?

QUESTION 4. Can every element of $G$ have trivial contraction group?

QUESTION 5. Can $G$ have an open subgroup with a nontrivial abelian normal subgroup?

Question 5 is answered negatively by Theorem A below. The first four questions are open questions to which we obtain negative answers under more restrictive hypotheses.

An example for Question 2 would also be an example for Question 1, since the normal closure of an infinite nonopen commensurated compact subgroup of $G$ would be a proper dense normal subgroup of $G$. We give several equivalent formulations of a negative answer to Question 2 in Theorems B and $C$ below.

By the recent groundbreaking work of Juschenko and Monod [32] and its extension due to Nekrashevych [45], there exist finitely generated infinite simple amenable groups, so the hypothesis in Question 3 that $G$ be nondiscrete is important. However, we emphasize that the question whether a group $G \in \mathscr{S}$ can be amenable is naturally linked with the investigation of the commensurated subgroups of finitely generated infinite simple amenable groups; see Remark 3.7 below.

Question 4 is a specialization of [70, Problem 4.1], which asks if $G \in \mathscr{S}$ can have the property that every element normalizes a compact open subgroup. By results of Baumgartner and Willis [7], given an element $g$ of a t.d.l.c. group $G$, then $g$ normalizes a compact open subgroup if and only if both $\overline{\operatorname{con}(g)}$ and $\overline{\operatorname{con}\left(g^{-1}\right)}$ are compact. (We refer to Section 1.7 below for the definition of contraction groups.) Thus an example for Question 4 would also be an example for [70, Problem 4.1].

The following basic example (a similar construction is given in [70, Proposition 3.2]) gives some additional motivation for the focus on compactly generated topologically simple groups in the questions above.

EXAMPLE 1.1. Let $S=\operatorname{Sym}(\mathbf{Z})$ be equipped with the permutation topology. Let $A=\operatorname{Alt}(\mathbf{Z})$ be the group of permutations of $\mathbf{Z}$ that can be written as a product of an even number of transpositions. Then $A$ is clearly a dense normal subgroup of $S$. It is well known that $\operatorname{Alt}(\mathbf{Z})$ is a simple group, which is easily seen by observing that it is the directed union of its simple subgroups isomorphic to $\operatorname{Alt}(n)$ for $n \geqslant 5$. 
Given $i \in \mathbf{Z}$, let $\sigma_{i}$ be the 3-cycle $(3 i 3 i+13 i+2)$ acting on $\mathbf{Z}$, and let $B$ be the smallest closed subgroup of $S$ containing $\left\{\sigma_{i} \mid i \in \mathbf{Z}\right\}$. We see that $B$ is an abelian profinite group of exponent 3 . Moreover, $B$ is commensurated by $A$. We can thus construct the nondiscrete t.d.l.c. group $G=\langle A, B\rangle$, equipped with the topology so that $B$ is embedded as a compact open subgroup.

Now observe the following: $G$ is topologically but not abstractly simple (since $A$ is a proper dense normal subgroup of $G$ ); $G$ is locally finite, hence amenable as an abstract group; every element of $G$ has trivial contraction group and open centralizer in $G$; the elements of $B$ that fix every negative integer form an infinite nonopen commensurated compact subgroup; and $B$ is an open abelian subgroup of $G$. So apart from the compact generation hypothesis, $G$ is an example for all five of the questions above.

1.2. Locally normal subgroups. Our approach in studying the class $\mathscr{S}$ is based on the concept of locally normal subgroup, that is a subgroup whose normalizer is open. One motivation for considering this concept is the following classical fact (see [11, Ch. III, Section 7, Proposition 2]): if $G$ is a $p$-adic Lie group, then a subalgebra of the $\mathbf{Q}_{p}$-Lie algebra of $G$ is an ideal if and only if it is the Lie algebra of a compact locally normal subgroup of $G$. Thus compact locally normal subgroups may be viewed as a group theoretic counterpart of ideals in Lie theory. In a general t.d.l.c. group $G$, obvious examples of compact locally normal subgroups are provided by the trivial subgroup, or by compact open subgroups; these locally normal subgroups should be considered as trivial.

The following theorem answers Question 5.

THEOREM A (See Theorem 5.3). Let G be a compactly generated t.d.l.c. group that is topologically characteristically simple. Suppose that $G$ is neither discrete nor compact. Then the following hold.

(i) No element of $G \backslash\{1\}$ has open centralizer in $G$.

(ii) The only virtually soluble locally normal subgroup of $G$ is the identity subgroup $\{1\}$.

Part (i) in Theorem A in the case of simple groups is [5, Theorem 4.8]; Part (ii) strengthens a result of Willis [70, Theorem 2.2].

REMARK 1.2. Independently, Wesolek [67] has given another generalization of [70, Theorem 2.2] and [5, Theorem 4.8] by obtaining a structure theory of second-countable t.d.l.c. groups that have a soluble open subgroup. 
Given Theorem A and the theory developed in [21], each $G \in \mathscr{S}$ admits three canonical bounded modular lattices (in the sense of partially ordered sets) arising from the arrangement of locally normal subgroups in $G$ :

The structure lattice $\mathcal{L N}(G)$ is defined to be the set of all closed locally normal subgroups, modulo the equivalence relation that $H \sim K$ if $H \cap K$ is open in $H$ and $K$, with ordering induced by inclusion of groups. We write 0 for the class of the trivial group and $\infty$ for the class of open subgroups.

The centralizer lattice $\mathcal{L C}(G)$ consists of all elements of $\mathcal{L N}(G)$ represented by the centralizer of a locally normal subgroup.

The local decomposition lattice consists of all elements of $\mathcal{L N}(G)$ represented by a direct factor of an open subgroup.

In general one has

$$
\{0, \infty\} \subseteq \mathcal{L D}(G) \subseteq \mathcal{L C}(G) \subseteq \mathcal{L N}(G)
$$

Note that all three lattices admit a natural action of $G$ by conjugation, with 0 and $\infty$ as fixed points. A point $\alpha \in \mathcal{L N}(G)$ is fixed by this action if and only if the compact representatives of $\alpha$ are commensurated by $G$, in other words, for every $g \in G$ the index $\left|K: K \cap g K^{-1}\right|$ is finite.

1.3. Fixed points in the structure lattice. Let us record three properties that, taken together, are equivalent to abstract simplicity of a nondiscrete $\sigma$-compact t.d.l.c. group $G$ (see Theorem 3.9).

(S0) $G$ has no proper open normal subgroups.

(S1) Every nontrivial normal subgroup of $G$ contains an infinite commensurated compact locally normal subgroup of $G$.

(S2) Every infinite commensurated compact subgroup of $G$ is open.

Nondiscrete topologically simple groups clearly satisfy (S0), so $G \in \mathscr{S}$ is abstractly simple if and only if it satisfies (S1) and (S2).

Question 2 asks, given $G \in \mathscr{S}$, if $G$ can fail to satisfy (S2). For a given $G \in \mathscr{S}$, there are several equivalent formulations of this question; in particular, we see some more necessary properties of abstractly simple groups in $\mathscr{S}$. Moreover, we can show that indeed $G$ has property (S2) under some additional hypotheses on $G$ (see Corollary 5.19).

TheOrem B (See Section 5.1). Let $G \in \mathscr{S}$. Then the following are equivalent.

(i) G satisfies (S2), that is, every infinite commensurated compact subgroup of $G$ is open. 
(ii) $G$ has no fixed points other than 0 and $\infty$ in its action on $\mathcal{L N}(G)$.

(iii) The only locally compact group topology that is a proper refinement of the given topology on $G$ is the discrete topology.

(iv) Given a nontrivial compact locally normal subgroup $H$ of $G$, then there exist $g_{1}, \ldots, g_{n} \in G$ such that

$$
G=\left\langle g_{1} H g_{1}^{-1}, g_{2} H g_{2}^{-1}, \ldots, g_{n} H g_{n}^{-1}\right\rangle .
$$

We obtain further equivalent formulations if we consider the class $\mathscr{S}$ as a whole. An $\overline{\mathrm{FC}}$-group is a topological group in which every conjugacy class has compact closure. The assertion (iv) in the next theorem is motivated by [18, Appendix II], and shows that Question 2 has more general significance for the structure of t.d.l.c. groups.

THEOREM C (See Section 5.5). The following assertions are equivalent.

(i) For all $G \in \mathscr{S}$, every infinite commensurated compact subgroup is open.

(ii) For all $G \in \mathscr{S}$, every commensurated open $\overline{\mathrm{FC}}$-subgroup is compact.

(iii) For all $G \in \mathscr{S}$, the set of compact open subgroups of $G$ is precisely the set of all open commensurated $\overline{\mathrm{FC}}$-subgroups of $G$.

(iv) Given a compactly generated t.d.l.c. group $G$ and distinct subgroups $N_{1}$ and $\mathrm{N}_{2}$ that are maximal among proper closed normal subgroups of $G$, then $N_{1} N_{2}=G$.

The next result shows that fixed points of $G$ acting on $\mathcal{L} \mathcal{N}(G)$ play an important role in the structure of orbits of $G$ on $\mathcal{L N}(G)$.

TheOrem D (See Theorem 5.2). Let $G \in \mathscr{S}$. For each $\alpha \in \mathcal{L} \mathcal{N}(G)$, there exist $g_{1}, \ldots, g_{n} \in G$ such that $g_{1} \alpha \vee \cdots \vee g_{n} \alpha$ is fixed by $G$.

The following corollary is clear.

Corollary E. Let $G \in \mathscr{S}$ and let $H$ be a normal subgroup of $G$. Suppose $H$ contains an infinite compact locally normal subgroup. Then $H$ contains an infinite commensurated compact locally normal subgroup. In particular, if every nontrivial normal subgroup of $G$ contains an infinite compact locally normal subgroup, then $G$ satisfies (S1). 
We also show that the set of nonzero fixed points of $G$ acting on $\mathcal{L N}(G)$ forms a filter (Lemma 5.11).

1.4. A partition of the class $\mathscr{S}$. The properties of the structure lattice, centralizer lattice and local decomposition lattice lead to a partition of $\mathscr{S}$ into five classes as follows:

TheOrem F (See Section 5.3). Let $G \in \mathscr{S}$. Then $G$ is of exactly one of the following types:

- locally h.j.i.: We have $\mathcal{L} \mathcal{N}(G)=\{0, \infty\}$, or equivalently, every compact open subgroup of $G$ is hereditarily just-infinite (h.j.i.), where a profinite group is said to be h.j.i. if every nontrivial closed locally normal subgroup is open.

- atomic type: $|\mathcal{L N}(G)|>2$ but $\mathcal{L C}(G)=\{0, \infty\}$, there is a unique least element of $\mathcal{L N}(G) \backslash\{0\}$, the action of $G$ on $\mathcal{L N}(G)$ is trivial and $G$ is not abstractly simple.

- nonprincipal filter type (abbreviated by NPF type): The set $\mathcal{L} \mathcal{N}(G) \backslash\{0\}$ is a nonprincipal filter in $\mathcal{L N}(G)$ and $\mathcal{L C}(G)=\{0, \infty\}$.

- (strictly) weakly decomposable: $|\mathcal{L C}(G)|>2$, but $\mathcal{L} \mathcal{D}(G)=\{0, \infty\}$.

- locally decomposable: $|\mathcal{L D}(G)|>2$.

Moreover, the type of $G$ is completely determined by the isomorphism type of $\mathcal{L N}(G)$ as a poset. In particular, if $G, H \in \mathscr{S}$ have isomorphic open subgroups then $G$ and $H$ are of the same type.

Other than locally h.j.i. groups, we do not know of any groups in $\mathscr{S}$ such that there is a h.j.i. compact locally normal subgroup or there is a minimal nonzero element of $\mathcal{L N}(G)$; the following puts further restrictions on the possible structure of such groups.

TheOREM G (See Section 6.6). Let $G \in \mathscr{S}$.

(i) Any hereditarily just-infinite compact locally normal subgroup is commensurated by $G$.

(ii) Suppose there is a minimal nonzero element of $\alpha$ of $\mathcal{L} \mathcal{N}(G)$. Then $G$ is either locally h.j.i. or of atomic type.

(iii) Suppose that $G$ is of atomic type. Then there exists $S \in \mathscr{S}$, unique up to isomorphism, and a continuous homomorphism $\phi: S \rightarrow G$ such that $S$ is not 
Table 1. Properties and open questions.

\begin{tabular}{cccccc}
\hline Property & Locally h.j.i. & Atomic & NPF & Weakly dec. & Locally dec. \\
\hline $\mathcal{L} \mathcal{N}$ & No & No & $?$ & Yes & Yes \\
$(\mathrm{S} 1)$ & Yes? & $?$ & $?$ & Yes & Yes \\
$(\mathrm{S} 2)$ & Yes & No & $?$ & Yes? & Yes \\
abstractly simple & Yes? & No & $?$ & Yes? & Yes \\
anisotropic & No? & $?$ & $?$ & No & No \\
amenable & No? & $?$ & $?$ & No & No \\
\hline
\end{tabular}

of atomic type and $\phi(S)$ is a proper dense normal subgroup of $G$ containing a representative of the atom of $\mathcal{L} \mathcal{N}(G)$. In particular, $G$ is not abstractly simple.

Table 1 below summarizes the progress we have made towards answering the first four questions given in the initial discussion. The following conventions are used in the table. We indicate both definitive results for all $G \in \mathscr{S}$ of the given type ('Yes' and 'No') and cases where no definitive result is known, but either all known examples satisfy the property or all known examples fail to satisfy the property ('Yes?' and 'No?'). There are no entries in the table for which some examples are known to satisfy the property and others are known not to satisfy it. We recall from Section 1.3 that $G \in \mathscr{S}$ is abstractly simple if and only if it satisfies (S1) and (S2), and that (S2) is equivalent to the property that the set of fixed points of $G$ acting on $\mathcal{L N}(G)$ is just $\{0, \infty\}$. In particular, a necessary condition for abstract simplicity is that either $G$ is locally h.j.i. or $G$ has faithful action on $\mathcal{L N}(G)$; we denote the latter by ' $\curvearrowright \mathcal{L N}$ ' in the table. We say $G$ is anisotropic if every element of $G$ has trivial contraction group.

Given $G \in \mathscr{S}$, there is a natural division between the case where $\mathcal{L C}(G)$ is trivial (corresponding to the first three types; an equivalent condition is that $\mathcal{L N}(G) \backslash\{0\}$ is closed under meets), and the case where $\mathcal{L C}(G)$ is nontrivial. In the latter case we obtain stronger results, and this case also includes most known examples. Indeed, the only known examples with trivial centralizer lattice are linear algebraic groups over local fields, which are locally h.j.i.; thus no examples at all are known in the atomic or NPF cases. We suspect that there does not exist a group in $\mathscr{S}$ of atomic type, since by Theorem $\mathrm{G}$ it would admit an embedding of a group in $\mathscr{S}$ of some other type as a proper dense normal subgroup. On the other hand, it could well be the case that some of the known examples of simple Kac-Moody groups are of NPF type. See Appendix A for a further discussion of the known examples. 
It seems that a major challenge in advancing our understanding of the class $\mathscr{S}$ is to construct simple groups with trivial centralizer lattice, or at least with trivial local decomposition lattice, that are significantly different from the ones we already know (see Remark 3.7 for a potential source of such examples).

1.5. Local composition factors. We next present some additional algebraic features of compact locally normal subgroups of groups in $\mathscr{S}$.

For any compactly generated t.d.l.c. group $G$, we observe (Proposition 4.6) that each compact open subgroup of $G / K$ has finitely many isomorphism types of composition factors, where $K$ is a compact normal subgroup that can be taken to lie in any given identity neighbourhood. This observation confirms a conjecture formulated in [69, Section 4] and naturally leads to the notion of the local prime content of $G / K$, which is the unique finite set $\eta=\eta(G / K)$ of primes such that every compact open subgroup of $G / K$ is virtually pro- $\eta$ and has an infinite pro- $p$ subgroup for each $p \in \eta$.

For groups in $G \in \mathscr{S}$, we obtain additional control over the local prime content of compact locally normal subgroups of $G$. In particular, the local prime content of $G$ can be recovered from any nontrivial locally normal subgroup. We can control the presence of nonabelian composition factors of compact open subgroups in a similar manner.

TheOREM H (See Theorem 4.14). Let $G \in \mathscr{S}$.

(i) If a nontrivial compact locally normal subgroup of $G$ is a pro- $\pi$ group for some set of primes $\pi$, then every compact open subgroup of $G$ is virtually pro- $\pi$. In particular, for all $p \in \eta(G)$, each closed locally normal subgroup $L \neq\{1\}$ has an infinite pro-p subgroup.

(ii) If a nontrivial compact locally normal subgroup of $G$ is prosoluble, then every compact open subgroup of $G$ is virtually prosoluble.

Theorem $\mathrm{H}$ was inspired by a result of Burger and Mozes, who obtained a similar statement in the case of locally primitive tree automorphism groups; see [14, Proposition 2.1.2].

1.6. Microsupported actions. An action of a group $G$ by homeomorphisms on a (possibly connected) topological space $X$ is called microsupported if for every nonempty open subset $Y$ of $X$, the pointwise stabilizer of the complement $X \backslash Y$ acts nontrivially on $Y$. A subset $V$ of $X$ is called compressible if it is nonempty and if for any nonempty open subset $Y$, there exists $g \in G$ such 
that $g V \subset Y$. Numerous natural transformation groups, like homeomorphism groups of various topological spaces (for example closed manifolds, the rationals, the Cantor space,...) or diffeomorphism groups of manifolds, happen to be microsupported and to have a compressible open set. This has been exploited repeatedly in the literature to show that many of those transformation groups are simple or almost simple (see Epstein's paper [26] and references therein), or to show reconstruction theorems (see Rubin's paper [56] and references Therein). (In [56, Definition 2.3], the term regionally disrigid is used to qualify what we have called a microsupported action.) A related result, known as Higman's simplicity criterion, valid for abstract groups, may be consulted in [9, Proposition C10.2].

The following result, whose short and self-contained proof will be provided in Section 6.2 below, provides a uniform explanation that the conjunction of these two properties naturally yields simple groups. A closely related statement (with formally stronger hypotheses) appears in [44, Proposition 4.3].

Proposition I. Let $G$ be a subgroup of the homeomorphism group of a Hausdorff topological space $X$. If the G-action is microsupported and has a compressible open set, then the intersection $M$ of all nontrivial normal subgroups of $G$ is nontrivial.

If in addition the $M$-action admits a compressible open set, then $M$ is simple.

It turns out that examples of transformation groups satisfying the hypotheses of Proposition I may also be found among nondiscrete t.d.l.c. groups. The prototypical case is provided by the action of the full automorphism group of a regular locally finite tree $T$ on the set of ends of $T$. The fact that the latter group is almost simple was first observed by Tits [62]; we refer to Appendix A for many other related examples of groups in $\mathscr{S}$.

Our next result shows that for groups $G \in \mathscr{S}$, all microsupported actions on compact totally disconnected spaces are controlled by the $G$-action on the centralizer lattice $\mathcal{L C}(G)$. Moreover, somewhat surprisingly, the existence of a compressible open set happens to be automatic for microsupported actions. In order to formulate precise statements, we recall that the lattices $\mathcal{L C}(G)$ and $\mathcal{L D}(G)$ are both Boolean algebras by [21, Theorem I]. By the Stone representation theorem, every Boolean algebra $\mathcal{A}$ is canonically isomorphic to the lattice of clopen sets of a profinite space, that is a compact zero-dimensional space, which is called the Stone space of $\mathcal{A}$, denoted by $\mathfrak{S}(\mathcal{A})$ and can be constructed as the set of ultrafilters on $\mathcal{A}$. We shall next see that the dynamics of the $G$-action on $\mathfrak{S}(\mathcal{L C}(G))$ is rich and can be exploited to shed light on the global algebraic properties of $G$. 
We also need to recall basic definitions from topological dynamics. An action of a locally compact group $G$ on a compact space $\Omega$ by homeomorphisms is called continuous if the corresponding homomorphism of $G$ to the homeomorphism group of $\Omega$, endowed with the topology of uniform convergence, is continuous. This implies that the $G$-action on $\Omega$ is continuous, that is the action map $G \times \Omega \rightarrow$ $\Omega$ is continuous. The $G$-action on $\Omega$ is called minimal if every orbit is dense. It is called strongly proximal if the closure of every $G$-orbit in the space of probability measures on $\Omega$, endowed with the weak-* topology, contains a Dirac mass. Suppose that $\Omega$ is totally disconnected and let $K$ be the kernel of the $G$ action. The action is called weakly decomposable (respectively locally weakly decomposable) if it is continuous and if for every clopen proper subset $\Omega^{\prime}$ of $\Omega$, the quotient $F / K$ of the pointwise stabilizer $F$ of $\Omega^{\prime}$ is nontrivial (respectively nondiscrete). Thus, for group actions on profinite spaces, 'microsupported' and 'weakly decomposable' are synonyms.

Theorem J (See Section 6.7). Let $G \in \mathscr{S}$ and let $\Omega$ be the Stone space associated with the centralizer lattice $\mathcal{L C}(G)$. Then:

(i) The $G$-action on $\Omega$ is continuous, minimal, strongly proximal and locally weakly decomposable; moreover $\Omega$ contains a compressible clopen subset.

(ii) Given a profinite space $X$ with a continuous $G$-action, the $G$-action on $X$ is microsupported if and only if there is a continuous $G$-equivariant surjective map $\Omega \rightarrow X$. In particular every continuous microsupported $G$-action on a profinite space is minimal, strongly proximal, locally weakly decomposable and has a compressible open set.

Notice that $\Omega=\mathfrak{S}(\mathcal{L C}(G))$ is a singleton if and only if $\mathcal{L C}(G)=\{0$, $\infty$. It should be emphasized that, although any group $G$ belonging to $\mathscr{S}$ is automatically second-countable (this follows from [33]), the centralizer lattice for $G \in \mathscr{S}$ is not necessarily countable, so $\Omega$ is not necessarily countably based. For example, one obtains an uncountable local decomposition lattice for t.d.l.c. groups $G$ such that $\mathrm{QZ}(G)=\{1\}$ and some compact open subgroup of $G$ splits as a direct product with infinitely many infinite factors: such a compact open subgroup thus has uncountably many direct factors, each of which represents a distinct class in $\mathcal{L D}(G)$. Examples of this kind arise as Burger-Mozes' universal groups acting on trees with local action prescribed by a suitable finite permutation group (see Appendix A).

Theorem $\mathrm{J}$ notably implies that $\mathcal{L C}(G) \neq\{0, \infty\}$ if and only if $G$ admits a continuous microsupported action on a profinite space containing more than one point. Whenever this is the case, Theorem $\mathrm{J}$ has several consequences on 
the structure of $G$, which we now proceed to describe. The first one relates to Question 3 and stems from the incompatibility between amenability and minimal strongly proximal actions:

Corollary K. Let $G \in \mathscr{S}$. Any closed cocompact amenable subgroup of $G$ fixes a point in $\Omega=\mathfrak{S}(\mathcal{L C}(G))$. In particular, if $\mathcal{L C}(G) \neq\{0, \infty\}$, then $G$ is not amenable, and if $G$ contains a closed cocompact amenable subgroup, then the $G$-action on $\Omega$ is transitive.

Here is another consequence of the dynamical properties highlighted in Theorem J, obtained by a standard ping-pong argument.

Corollary L. Let $G \in \mathscr{S}$ be such that $\mathcal{L C}(G) \neq\{0, \infty\}$. Then $G$ contains a nonabelian discrete free subsemigroup.

We recall that a Polish group is a topological group whose underlying topology is homeomorphic to a separable complete metric space. Every second-countable locally compact group is a Polish group, but not conversely. Another consequence of Theorem $\mathrm{J}$ is that we can apply results from [21] to obtain topological rigidity results for groups in $\mathscr{S}$ :

Corollary M. Let $G \in \mathscr{S}$. If $\mathcal{L C}(G) \neq\{0, \infty\}$, then the topology of $G$ is the unique $\sigma$-compact locally compact group topology on $G$ and the unique Polish group topology on $G$. In particular every automorphism of $G$ is continuous.

If in addition $G$ is abstractly simple (for instance if $\mathcal{L D}(G) \neq\{0, \infty\}$ by Theorem $Q$ below), then there are exactly two locally compact group topologies on $G$, namely the original one and the discrete topology.

Notice that the corresponding result does not hold for all simple Lie groups, since the field of complex numbers admits discontinuous automorphisms; we refer to [35] for a comprehensive statement in the case of semisimple Lie groups.

1.7. Contraction groups and abstract simplicity. Let $G$ be topological group. Recall that the contraction group of $g$ is defined by

$$
\operatorname{con}(g)=\left\{x \in G \mid \lim _{n \rightarrow \infty} g^{n} x g^{-n}=e\right\} .
$$

In every known example of $G \in \mathscr{S}$, some element of $G$ has nontrivial contraction group; whether this is true for all $G \in \mathscr{S}$ is Question 4 above.

In simple Lie groups or in simple algebraic groups over local fields, the contraction group of every element is known to coincide with the unipotent radical 
of some parabolic subgroup, and is thus always closed. On the other hand, recent results indicate that many nonlinear examples of groups in $\mathscr{S}$ have nonclosed contraction groups (see [6] and [40]), while in some specific cases the closedness of contraction groups yields connections with finer algebraic structures related to linear algebraic groups (see [17]).

As a partial answer to Question 4, we prove the following:

Theorem N (See Section 6.6). Let $G \in \mathscr{S}$. Then $\mathcal{L C}(G) \neq\{0, \infty\}$ if and only if $G$ has a closed subgroup of the form $R=\prod_{i \in \mathbf{Z}} K_{i} \rtimes\langle g\rangle$, where $K_{i}$ is an infinite compact locally normal subgroup of $G$ and $g$ acts by shifting indices.

Corollary O. Let $G \in \mathscr{S}$. If $\mathcal{L C}(G) \neq\{0, \infty\}$, then the contraction group of some element of $G$ is not closed (and hence nontrivial).

In relation with the question of abstract simplicity, we observe that the combination of Proposition I and Theorem J directly implies that a group in $\mathscr{S}$ with a nontrivial centralizer lattice has a smallest dense normal subgroup which is abstractly simple. Below, we shall obtain an alternative proof of the following stronger statements by combining the main result of [20] with the conclusions of Corollary $\mathrm{O}$.

TheOrem P (See Section 6.8). Let $G \in \mathscr{S}$. Suppose that at least one of the following holds:

(i) $\mathcal{L C}(G) \neq\{0, \infty\}$;

(ii) Some compact open subgroup of $G$ is finitely generated as a profinite group.

Then $G$ has property (S1). Consequently, $G$ is abstractly simple if and only if every nontrivial commensurated compact subgroup of $G$ is open.

TheOREM Q (See Section 6.8). Let $G \in \mathscr{S}$. Suppose that at least one of the following holds:

(i) $\mathcal{L} \mathcal{D}(G) \neq\{0, \infty\}$;

(ii) There is a compact open subgroup $U$ of $G$ such that $U$ is finitely generated as a profinite group and $\overline{[U, U]}$ is open in $G$;

(iii) Some nontrivial compact locally normal subgroup of $G$ is finitely generated as a profinite group and every infinite commensurated compact subgroup of $G$ is open.

Then $G$ is abstractly simple. 
1.8. Abstract linearity. A locally compact group $G$ is called linear if there exist an integer $d>0$, a locally compact field $k$ and a continuous injective homomorphism $\varphi: G \rightarrow \mathrm{GL}_{d}(k)$. The group $G$ is called abstractly linear if the underlying abstract group of $G$ is linear. It has been shown in [22, Corollary 1.6] (relying in part on the results of the present paper) that the only compactly generated topologically simple linear locally compact groups are the finite simple groups, the simple Lie groups and the simple algebraic groups over local fields. It is an open question to determine whether the same result holds if one only requires abstract linearity. In this direction, we point out the following consequence of Theorem N.

Corollary R (See Section 6.6). Let $G \in \mathscr{S}$. If $\mathcal{L C}(G) \neq\{0, \infty\}$, then $G$ contains a finitely generated subgroup which is not residually finite. In particular $G$ is not abstractly linear over any field.

\section{Preliminaries}

In this section we recall some definitions and results from [18], [21] and [20] that will be used below.

\subsection{Open compact subgroups and locally normal subgroups.}

DEFINITION 2.1. Let $G$ be a totally disconnected, locally compact (t.d.l.c.) group. Write $\mathcal{B}(G)$ for the set of compact open subgroups of $G$, and let $U \in \mathcal{B}(G)$.

Let $H$ be a subgroup of $G$. We say that $H$ is commensurate to a subgroup $K \leqslant G$ if the indices $[H: H \cap K]$ and $[K: H \cap K]$ are both finite. Write $[H]$ for the class of all subgroups $K$ of $G$ such that $K \cap U$ is commensurate with $H \cap U$. Say $H$ and $K$ are locally equivalent if $[H]=[K]$. There is a natural partial ordering on local equivalence classes, given by $[H] \geqslant[K]$ if $H \cap K \cap U$ is commensurate with $K \cap U$.

A locally normal subgroup of $G$ is a subgroup $H$ such that $\mathrm{N}_{G}(H)$ is open. The structure lattice $\mathcal{L} \mathcal{N}(G)$ of $G$ is the set of local equivalence classes of closed locally normal subgroups of $G$.

The partial order on local equivalence classes given in Definition 2.1 may be reformulated, for $\alpha, \beta \in \mathcal{L N}(G)$, as $\alpha \leqslant \beta$ if $H \leqslant K$ for some $H \in \alpha$ and $K \in \beta$.

As noted in [21], the structure lattice is a modular lattice, admitting an action of the group $\operatorname{Aut}(G)$ of topological group automorphisms by conjugation, and is a local invariant of the group in that $\mathcal{L N}(G)=\mathcal{L} \mathcal{N}(H)$ for any open subgroup $H$ of $G$. If $G$ is not discrete, there are two 'trivial' elements of $\mathcal{L N}(G)$, namely the 
class of the trivial group, which we denote 0 , and the class of the compact open subgroups, which we denote $\infty$. More generally the symbols 0 and $\infty$ will be used to denote the minimum and maximum elements respectively of a bounded lattice. When discussing subsets of $\mathcal{L N}(G)$, we regard a subset as nontrivial if it contains an element other than 0 and $\infty$.

2.2. Topological countability. A topological space is $\sigma$-compact if it is a union of countably many compact subspaces; first-countable if at every point, there is a countable base of neighbourhoods; and second-countable if there is a countable base for the topology.

The following well-known facts (the first two of which are easily verified) will for the most part allow us to restrict attention to totally disconnected locally compact second-countable (t.d.l.c.s.c.) groups.

LEMMA 2.2. Let $G$ be a t.d.l.c. group.

(i) $G$ is second-countable if and only if it is $\sigma$-compact and first-countable.

(ii) If $G$ is compactly generated, then $G$ is $\sigma$-compact.

(iii) (See [33]) If $G$ is $\sigma$-compact, then for every identity neighbourhood $U$ in $G$, there exists $K \subseteq U$ such that $K$ is a compact normal subgroup of $G$ and $G / K$ is second-countable.

We note in particular the following.

COROllary 2.3. Every $G \in \mathscr{S}$ is second-countable.

There is one more lemma from [21] concerning first-countable groups that we use frequently.

LEMMA 2.4 [21, Lemma 7.4]. Let $U$ be a first-countable profinite group and let $K$ be a closed subgroup of $U$. Then $U$ commensurates $K$ if and only if $U$ normalizes an open subgroup of $K$.

\subsection{Quasicentralizers.}

DEFINITION 2.5. Let $G$ be a t.d.l.c. group and let $H$ and $K$ be subgroups of $G$. The quasicentralizer of $K$ in $H$ is given by

$$
\mathrm{QC}_{H}(K):=\bigcup_{U \in \mathcal{B}(G)} \mathrm{C}_{H}(K \cap U),
$$


where $\mathcal{B}(G)$ is the set of compact open subgroups of $G$. Notice that the quasicentralizer $\mathrm{QC}_{H}(K)$ depends only on the local equivalence class of $K$; we shall therefore use the notation

$$
\mathrm{QC}_{H}([K])=\mathrm{QC}_{H}(K) \text {. }
$$

If the class $[K]$ is fixed under the conjugation action of $H$, then the quasicentralizer $\mathrm{QC}_{H}(K)$ is normal in $H$. A typical example is provided by the quasicentre $\mathrm{QZ}(H)$ of $H$, which is just $\mathrm{QC}_{H}(H)$.

Definition 2.6. Let $G$ be a t.d.l.c. group. A closed subgroup $H$ is $\mathbf{C}$-stable in $G$ if $\mathrm{QC}_{G}(H) \cap \mathrm{QC}_{G}\left(\mathrm{C}_{G}(H)\right)$ is discrete. Say a t.d.l.c. group $G$ is locally $\mathbf{C}$-stable if all closed locally normal subgroups of $G$ are $\mathrm{C}$-stable in $G$.

Proposition 2.7 (See [21, Theorem 3.19]). Let $G$ be a t.d.l.c. group. Then $G$ is locally $C$-stable if and only if $\mathrm{QZ}(G)$ is discrete and every nontrivial abelian compact locally normal subgroup of $G$ is contained in $\mathrm{QZ}(G)$.

If in addition $\mathrm{QZ}(G)=\{1\}$, then for every closed locally normal subgroup $H$ of $G$, we have $\mathrm{QZ}(H)=\{1\}$ and $\mathrm{QC}_{G}(H)=\mathrm{C}_{G}(H)$.

\subsection{Local decomposition.}

DEFINITION 2.8. Let $G$ be a t.d.l.c. group such that $\mathrm{QZ}(G)=\{1\}$. Define the local decomposition lattice $\mathcal{L D}(G)$ of $G$ to be the subset of $\mathcal{L N}(G)$ consisting of elements $[K]$ where $K$ is a closed direct factor of some compact open subgroup of $G$. The complementation map on $\mathcal{L D}(G)$ sends $[K]$ to the unique $\alpha \in \mathcal{L D}(G)$ such that there is a representative $L$ of $\alpha$ for which $K \cap L=\{1\}$ and $K L$ is open.

DEFinition 2.9. Let $G$ be a locally C-stable t.d.l.c. group. Define the map $\perp$ : $\mathcal{L N}(G) \rightarrow \mathcal{L N}(G)$ to be given by $\alpha \mapsto\left[\mathrm{QC}_{G}(\alpha)\right]$. Define the centralizer lattice $\mathcal{L C}(G)$ be the set $\left\{\alpha^{\perp} \mid \alpha \in \mathcal{L N}(G)\right\}$ together with the map $\perp$ restricted to $\mathcal{L C}(G)$, partial order inherited from $\mathcal{L N}(G)$ and binary operations $\wedge_{c}$ and $\vee_{c}$ given by:

$$
\begin{gathered}
\alpha \wedge_{c} \beta=\alpha \wedge \beta \\
\alpha \vee_{c} \beta=\left(\alpha^{\perp} \wedge \beta^{\perp}\right)^{\perp} .
\end{gathered}
$$

In general we write $\vee$ instead of $\vee_{c}$ in contexts where it is clear that we are working inside the centralizer lattice.

THEOREM 2.10 [21, Theorems 4.5 and 5.2]. Let $G$ be a t.d.l.c. group. 
(i) Suppose that $G$ has trivial quasicentre. Then $\mathcal{L D}(G)$ is a Boolean algebra and $\perp$ induces the complementation map on $\mathcal{L D}(G)$.

(ii) Suppose in addition that $G$ has no nontrivial compact abelian locally normal subgroups. Then $\mathcal{L C}(G)$ is a Boolean algebra, $\perp$ induces the complementation map on $\mathcal{L C}(G)$ and $\mathcal{L D}(G)$ is a subalgebra of $\mathcal{L C}(G)$.

2.5. Decomposing locally compact groups into simple pieces. In this article, we adopt the general convention that a maximal subgroup of the group $G$ is one that is maximal among the proper subgroups of $G$, and a minimal subgroup is one that is minimal among the nontrivial subgroups of $G$. A similar convention applies when discussing more restrictive classes of subgroups, such as closed normal subgroups.

Topologically simple groups appear naturally in the upper structure of compactly generated t.d.l.c. groups. The following is an analogue of the wellknown fact that a nontrivial finitely generated group has a simple quotient.

THEOREM 2.11 [18, Theorem A]. Let $G$ be a compactly generated locally compact group. Then exactly one of the following holds.

(i) G has an infinite discrete quotient.

(ii) G has a cocompact closed normal subgroup that is connected and soluble.

(iii) $G$ has a cocompact closed normal subgroup $N$ such that $N$ has no infinite discrete quotient, but $N$ has exactly $n$ noncompact topologically simple quotients, where $0<n<\infty$.

In particular, Theorem 2.11 provides information on maximal closed normal subgroups of $G$. The following related result is concerned with minimal ones. A version of this result was asserted as part of [18, Proposition 2.6]; however, the statement and proof in the original published version contained an error. To avoid confusion, we give a proof here of a corrected version of the proposition that will be sufficient for the purposes of the present paper.

Proposition 2.12. Let $G$ be a compactly generated t.d.l.c. group which possesses no nontrivial discrete or abelian normal subgroup, and such that there exists an open subgroup $U$ of $G$ for which $\bigcap_{g \in G} g U g^{-1}=\{1\}$. Then every nontrivial closed normal subgroup of $G$ contains a minimal one, and the set $\mathcal{M}$ of minimal closed normal subgroups is finite. 
Proof. By [2] (see also Proposition 4.1 below), there is a locally finite connected graph $\Gamma$ on which $G$ has a continuous vertex-transitive proper action. The hypothesis that an open subgroup of $G$ has trivial core ensures that we can choose $\Gamma$ so that $G$ acts faithfully; the hypothesis that $G$ has no nontrivial discrete normal subgroup ensures that no nontrivial normal subgroup acts freely on $\Gamma$. Fix $v \in V \Gamma$ and write $N(v)$ for all vertices in $\Gamma$ at distance at most 1 from $v$. Let $A$ be the stabilizer of $v$ in $G$ and let $B$ be the subgroup fixing pointwise the set $N(v)$. Note that the assumptions on the action ensure that $A$ is a compact subgroup of $G$ and $B$ is an open subgroup of $A$.

Let $\mathcal{C}$ be a family of nontrivial closed normal subgroups of $G$. Suppose that $\mathcal{C}$ is filtering, that is, for any finite subset $\left\{K_{1}, \ldots, K_{n}\right\}$ of $\mathcal{C}$, there exists $K \in \mathcal{C}$ such that $K \leqslant \bigcap_{i=1}^{n} K_{i}$. We claim that $\mathcal{C}$ has nontrivial intersection.

Let $K \in \mathcal{C}$. Since $K$ acts faithfully but not freely on $\Gamma$, there exist adjacent vertices $w, x \in V \Gamma$ and $L \leqslant K$ such that $L$ fixes $x$ but not $w$. Since $G$ acts vertex-transitively and $K$ is normal, by conjugating in $G$ we can assume $x=v$ and take $L=K \cap A$; since $L$ does not fix $w$ we have $K \cap A \not \leq B$. In particular, we see that $K$ has nonempty intersection with the compact set $A \backslash B$. If we let $R=\bigcap_{K \in \mathcal{C}} K$, it now follows by compactness that $R \cap A \not \leq B$, so in particular $R$ is nontrivial and the claim is proved.

Since $\mathcal{C}$ can in particular be any chain of nontrivial closed normal subgroups of $G$, we conclude by Zorn's lemma that every nontrivial closed normal subgroup of $G$ contains a minimal one.

It remains to suppose that the set $\mathcal{M}$ of minimal closed normal subgroups is infinite and obtain a contradiction. Let $\mathcal{F}$ be the set of finite subsets of $\mathcal{M}$. For each $F \in \mathcal{F}$, let $M_{F}=\overline{\langle M \in \mathcal{M} \mid M \notin F\rangle}$. Notice that distinct minimal closed normal subgroups of $G$ commute; since centralizers are closed, it follows that $M_{F} \leqslant \mathrm{C}_{G}(M)$ for all $M \in F$. Now $\mathcal{C}=\left\{M_{F} \mid F \in \mathcal{F}\right\}$ is a filtering family of nontrivial closed normal subgroups of $G$, so the intersection $S=\bigcap_{F \in \mathcal{F}} M_{F}$ is nontrivial. We see that $S \leqslant \mathrm{C}_{G}(M)$ for all $M \in F$ and all $F \in \mathcal{F}$, so that $S \leqslant \mathrm{C}_{G}(M)$ for all $M \in \mathcal{M}$, and yet $S$ is in the closed subgroup generated by the elements of $\mathcal{M}$. It follows that $S$ is a nontrivial abelian normal subgroup of $G$, giving the required contradiction.

\section{Connections between topological and abstract simplicity}

Our focus in this paper is on t.d.l.c. groups that have restrictions imposed on their normal subgroups; in particular, our methods apply to the class of compactly generated topologically simple t.d.l.c. groups. A recurring theme is the difference between topological simplicity and abstract simplicity; indeed, as pointed out in 
the introduction, it is not known whether there are any compactly generated t.d.l.c. groups that are topologically but not abstractly simple.

Our standard assumption is that the group we are working with is a compactly generated, topologically simple t.d.l.c. group. However, the condition of topological simplicity can sometimes be relaxed, and we do so where this is convenient. In addition, in some cases results will pertain not just to the given t.d.l.c. group, but to any sufficiently large subgroup (for instance dense subgroups). Such results are of interest outside the theory of t.d.l.c. groups, as there is a strong connection between dense subgroups of t.d.l.c. groups and the theory of Hecke pairs, that is, groups with a specified commensurated subgroup. (For a general discussion of this connection, see [49].) An example of this connection is given by Proposition 3.6 below.

\subsection{Dense embeddings into topologically simple t.d.l.c. groups.}

Definition 3.1. A Hecke pair of groups $(\Gamma, \Delta)$ is a group $\Gamma$, together with a subgroup $\Delta$ such that $g \Delta g^{-1}$ is commensurate with $\Delta$ for all $g \in \Gamma$.

Each t.d.l.c. group $G$ and compact open subgroup $U$ form a Hecke pair. Conversely, each Hecke pair $(\Gamma, \Delta)$ canonically determines two totally disconnected groups and compact open subgroups.

THEOREM 3.2 ([8, Theorem 7.1]; [49, Theorem 1.6]). Let $(\Gamma, \Delta)$ be a Hecke pair of groups. Then there are t.d.l.c. groups and homomorphisms

$$
\beta_{\Delta}: \Gamma \rightarrow \hat{\Gamma}_{\Delta} \text { and } \beta_{\Gamma / / \Delta}: \Gamma \rightarrow \Gamma / / \Delta
$$

with the following properties.

(i) $\Delta$ is the inverse image under $\beta_{\Delta}$ (respectively $\beta_{\Gamma / / \Delta}$ ) of a compact open subgroup $U \leqslant \hat{\Gamma}_{\Delta}$ (respectively $U \leqslant \Gamma / / \Delta$ ).

(ii) Given any t.d.l.c. group $H$ and homomorphism $\beta: \Gamma \rightarrow H$ with dense image such that $\Delta=\beta^{-1}(U)$ for some compact open subgroup $U$ of $H$, there are unique continuous quotient maps $\psi_{1}: \hat{\Gamma}_{\Delta} \rightarrow H$ and $\psi_{2}: H \rightarrow \Gamma / / \Delta$ with compact kernels such that the following diagram commutes.

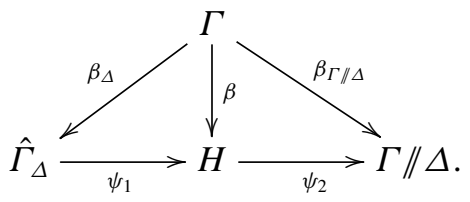


The maps $\beta_{\Delta}$ and $\beta_{\Gamma / / \Delta}$ are called respectively the Belyaev and Schlichting completions of the Hecke pair $(\Gamma, \Delta)$. The Belyaev completion has the additional property ([49, Theorem 1.5]) that any homomorphism $\phi: \Gamma \rightarrow H$ such that the closure of $\phi(\Delta)$ in $H$ is profinite lifts to a homomorphism $\hat{\phi}: \hat{\Gamma}_{\Delta} \rightarrow H$ such that $\phi=\hat{\phi} \circ \beta_{\Delta}$. Moreover, $\hat{\phi}$ is a quotient map if and only if $\phi(\Gamma)$ is dense and the closure of $\phi(\Delta)$ in $H$ is open. If $H$ has no compact normal subgroups, then $\operatorname{Ker} \hat{\phi}$ contains the compact kernel of the quotient map to $\Gamma / / \Delta$ and $\phi$ factors through the Schlichting completion. If $\hat{\phi}$ is a quotient map, then $\bar{\phi}$ is as well, that is, $H$ is a quotient of $\Gamma / / \Delta$.

The Belyaev and Schlichting completions of $(\Gamma, \Delta)$ need not be topologically simple even if $\Gamma$ is. The Schlichting completion of the Hecke pair $\left(\operatorname{PSL}_{n}(\mathbf{Q})\right.$, $\mathrm{PSL}_{n}(\mathbf{Z})$ ), for instance, has a closed normal subgroup isomorphic to $\operatorname{PSL}_{n}\left(\mathbf{Q}_{p}\right)$ for every prime $p$. Proposition 3.6 implies that a finite number of simple quotients may be recovered nevertheless when $\Gamma$ is finitely generated. The argument requires, not simplicity, but only a condition on how $\Gamma$ embeds into its completion and that condition will be introduced first. The condition is based in the following ideas, which also recur in later sections.

Let $G$ be a topological group. The monolith $\operatorname{Mon}(G)$ is the intersection of all nontrivial closed normal subgroups of $G$, see [18], and $G$ is said to be monolithic if $\operatorname{Mon}(G)>1$. If $G$ is monolithic then its monolith is topologically characteristically simple; conversely, if $G$ is a topologically characteristically simple t.d.l.c. group, then $G \rtimes \operatorname{Aut}(G)$ (with $G$ embedded as an open subgroup) is monolithic. The following consequence of Proposition 2.12 illustrates that monolithic groups appear naturally in the structure of general t.d.l.c. groups.

COROLLARY 3.3. Let $G$ be a compactly generated t.d.l.c. group which possesses no nontrivial discrete, compact or abelian normal subgroup, and let $\mathcal{M}$ be as in Proposition 2.12. Then for any $M \in \mathcal{M}$, the quotient $G / \mathrm{C}_{G}(M)$ has monolith $\overline{M \mathrm{C}_{G}(M)} / \mathrm{C}_{G}(M)>1$.

Proof. Let $N$ be the preimage in $G$ of a nontrivial closed normal subgroup of the quotient $G / \mathrm{C}_{G}(M)$. Then $N$ contains properly $\mathrm{C}_{G}(M)$. In particular $N$ does not commute with $M$, and so $N \cap M$ is nontrivial. By the minimality of $M$, this implies that $N$ contains $M$. Hence $N$ contains the closure $\overline{M C_{G}(M)}$. Thus every nontrivial closed normal subgroup of $G / \mathrm{C}_{G}(M)$ contains the closure of the image of $M$. It remains to check that $M$ has nontrivial image in the quotient $G / \mathrm{C}_{G}(M)$. This is indeed the case, since $M$ is nonabelian and thus is not contained in its own centralizer.

By definition, the image of the monolith $\operatorname{Mon}(G)$ in any quotient of $G$ is either injective or trivial. Our purposes require to consider other subgroups $\Gamma \leqslant G$ 
enjoying that property. We say that $\Gamma$ is relatively simple in $G$ if for every closed normal subgroup $N$ of $G$ the map $\Gamma \rightarrow G / N$ is either injective or trivial. We say that $\Gamma$ is relatively just-infinite in $G$ if for every closed normal subgroup $N$ of $G$ the map $\Gamma \rightarrow G / N$ is either injective or has finite image.

Clearly, if $\Gamma$ or $G$ is topologically simple (respectively just-infinite), then $\Gamma$ is relatively simple (respectively relatively just-infinite) in $G$. The next proposition, whose straightforward proof is omitted, implies that the converse need not hold.

PROPOSITION 3.4. Let $\Gamma \leqslant G$ be relatively simple (respectively relatively justinfinite). Then every nontrivial subgroup of $\Gamma$ is relatively simple (respectively relatively just-infinite) in G. Moreover, for every closed normal subgroup $N$ of $G$, the quotient group $\Gamma N / N$ is relatively simple (respectively relatively just-infinite) in $G / N$.

Since the group $\mathrm{PSL}_{n}(\mathbf{Q})$ is simple, it is a relatively simple subgroup of its Schlichting completion of $\left(\mathrm{PSL}_{n}(\mathbf{Q}), \mathrm{PSL}_{n}(\mathbf{Z})\right)$. Therefore, by Proposition 3.4, so are the embeddings of the subgroups $\operatorname{PSL}_{n}(\mathbf{Z}[1 / m])$ for each $m>0$ even though they are not simple. We are interested in the general case when the group $\Gamma$ embeds as a relatively simple or relatively just-infinite subgroup of the Belyaev or Schlichting completions of $(\Gamma, \Delta)$; in this case the embedding has the additional property of being dense.

LEMMA 3.5. Let $G$ be a nondiscrete t.d.l.c. group with a dense subgroup $\Gamma$.

If $\Gamma$ is relatively simple (respectively relatively just-infinite), then every discrete quotient of $G$ is trivial (respectively finite).

Proof. Let $O$ be an open normal subgroup of $G$. Then $O \cap \Gamma>1$ since $\Gamma$ is dense and $G$ is nondiscrete. Hence the image of $\Gamma$ in $G / O$ is not injective, and is thus trivial (respectively finite). The result follows since $\Gamma$ is dense, and thus has dense image in $G / O$.

PROPOSITION 3.6. Let $\Gamma$ be a group with an infinite commensurated subgroup $\Delta<\Gamma$ of infinite index. Suppose that $\Gamma$ is generated by finitely many cosets of that subgroup (for example $\Gamma$ is finitely generated).

(i) If $\Gamma$ is just-infinite, then $\Gamma$ embeds as a dense subgroup of some compactly generated monolithic t.d.l.c. group $G$ whose monolith is cocompact and a quasiproduct of finitely many pairwise isomorphic groups in $\mathscr{S}$ (we refer to [18] for the definition of a quasiproduct, a notion which will not be used anywhere else in the present paper). 
(ii) If $\Gamma$ is hereditarily just-infinite, then a finite index subgroup of $\Gamma$ embeds as a dense subgroup of some compactly generated monolithic t.d.l.c. group $G$ whose monolith is cocompact and belongs to $\mathscr{S}$.

(iii) If $\Gamma$ is simple, then $\Gamma$ embeds as a dense subgroup of some group $G \in \mathscr{S}$.

Proof. We assume that $\Gamma$ is just-infinite. Then Theorem 3.2 yields a nondiscrete t.d.l.c. group $H=\hat{\Gamma}_{\Delta}$ with a dense embedding of $\Gamma$ as a relatively just-infinite subgroup. Moreover, since $\Gamma$ is generated by finitely many cosets of $\Delta$, we see that $H$ is compactly generated, and since $\Delta$ is of infinite index in $\Gamma$, the group $H$ is not compact. By [18, Proposition 5.2], the group $H$ has a closed normal subgroup $Q$ such that the quotient $H / Q$ is not compact, and moreover every closed normal subgroup of $H$ properly containing $Q$ is cocompact (in the terminology of [18], the group $H / Q$ is just-noncompact). By Lemma 3.5, the group $H$ has no infinite discrete quotient. In particular $H / Q$ is nondiscrete. Since $H / Q$ is totally disconnected and noncompact, it does not have a connected cocompact normal subgroup either. Therefore, [18, Theorem E] ensures that $H / Q$ is a monolithic group whose monolith is the quasiproduct of finitely many pairwise isomorphic groups in $\mathscr{S}$. This proves (i).

Let now $H_{1}$ be the finite index open normal subgroup of $H$ containing $Q$ which is the kernel of the $H$-action on the quasifactors $N_{1} / Q, \ldots, N_{n} / Q$ of the monolith of $H / Q$. Since the quasifactors commute pairwise, we have $N_{i} \leqslant H_{1}$ for all $i$. Moreover, it follows from Corollary 3.3 that $G=H_{1} / \mathrm{C}_{H_{1}}\left(N_{1}\right)$ is monolithic, whose monolith is cocompact and belongs to $\mathscr{S}$. Assuming now in addition that $\Gamma$ is hereditarily just-infinite, we see that the intersection $\Gamma_{1}=H_{1} \cap \Gamma$ has finite index in $\Gamma$, and is thus just-infinite and dense in $H_{1}$. Therefore, $\Gamma_{1}$ is relatively just-infinite in $H_{1}$, and thus maps injectively onto a dense subgroup of $G$. This proves (ii).

Finally, in case $\Gamma$ is simple, every finite quotient of $\Gamma$ is trivial, which implies that every profinite quotient of $H$ is trivial as well. This forces the just-noncompact group $H / Q$ to be topologically simple and, hence, to belong to $\mathscr{S}$.

Proposition 3.6 is well illustrated by the Hecke pair $\left(\operatorname{PSL}_{n}(\mathbf{Z}[1 / m]), \operatorname{PSL}_{n}(\mathbf{Z})\right)$. It may be verified, for any $m>1$, that $\operatorname{PSL}_{n}(\mathbf{Z}[1 / m])$ is generated by finitely many cosets of $\operatorname{PSL}_{n}(\mathbf{Z})$ and, moreover, $\mathrm{PSL}_{n}(\mathbf{Z}[1 / m])$ is embedded, for any set of primes $\pi$, as a relatively simple subgroup in $\prod_{p \in \pi} \operatorname{PSL}_{n}\left(\mathbf{Q}_{p}\right)$ by restricting the embedding of the simple group $\operatorname{PSL}_{n}(\mathbf{Q})$. This embedding of $\operatorname{PSL}_{n}(\mathbf{Z}[1 / m])$ in $\prod_{p \in \pi} \operatorname{PSL}_{n}\left(\mathbf{Q}_{p}\right)$ is dense if and only if $\pi$ is a set of prime divisors of $m$. The topologically simple groups afforded by Proposition 3.6 are then the groups $\operatorname{PSL}_{n}\left(\mathbf{Q}_{p}\right), p \in \pi$. 
Proposition 3.6(ii) highlights the relevance of the study of the class $\mathscr{S}$ to the study of commensurated subgroups of hereditarily just-infinite groups. In particular the investigation of $\mathscr{S}$ is relevant to the Margulis-Zimmer conjecture according to which a lattice in a connected centreless simple Lie group of rank $\geqslant 2$ has no commensurated subgroup other than the finite or the finite index subgroups (see [58] for a thorough discussion of that conjecture as well as partial results on nonuniform lattices).

REMARK 3.7. Proposition 3.6 is also a potential source of new topologically simple t.d.l.c. groups. After all, we have seen that the well-known groups $\operatorname{PSL}_{n}\left(\mathbf{Q}_{p}\right)$ arise in this way. Should there exist a finitely generated, simple, amenable group with an infinite and proper commensurated subgroup, then this construction will produce an amenable group in the class $\mathscr{S}$. Similarly, should there exist a finitely generated simple group $\Gamma$ with an infinite and proper commensurated subgroup $\Delta$ such that every element of $\Gamma$ has finite order, then there would be an anisotropic group in the class $\mathscr{S}$.

REMARK 3.8. A variation on Proposition 3.6 was recently used by Wesolek in combination with the normal subgroup structure theory of t.d.l.c. groups to show that in a finitely generated just-infinite branch group, every commensurated subgroup is either finite or of finite index (see [68]).

3.2. Characterizing abstract simplicity in $\sigma$-compact t.d.l.c. groups. The main result of this section characterizes abstract simplicity of $\sigma$-compact t.d.l.c. groups. We return to the named properties (S1) and (S2) later in the article.

THEOREM 3.9. Let $G$ be a nondiscrete $\sigma$-compact t.d.l.c. group. Then $G$ is abstractly simple if and only if it has the following three properties:

(S0) G has no proper open normal subgroups.

(S1) Every nontrivial normal subgroup of $G$ contains an infinite commensurated compact locally normal subgroup of $G$.

(S2) Every infinite commensurated compact subgroup of $G$ is open.

If $G$ is abstractly simple then the following stronger version of (S2) holds:

$\left(\mathrm{S} 2^{\prime}\right)$ Suppose $U$ is an open subgroup of $G$, and $K$ is an infinite normal subgroup of $U$ (not necessarily closed) that is commensurated by $G$. Then $K$ has countably many cosets in $G$ and $\bar{K}$ is an open subgroup of $G$.

Given a nondiscrete t.d.l.c. group $G$, it is obvious that (S0), (S1) and (S2) together imply abstract simplicity of $G$, and that (S0) and (S1) are necessary for abstract simplicity. The nontrivial part of the proof is to show that (S2) is 
also necessary when $G$ is second-countable. In particular, it will follow that given $G \in \mathscr{S}$, then $G$ is abstractly simple if and only if it satisfies both (S1) and (S2).

Let us first recall that by Lemma 2.4, given any first-countable profinite group $U$ and closed subgroup $K$ of $U$, then $U$ commensurates $K$ if and only if $U$ normalizes an open subgroup of $K$. In other words:

LEMMA 3.10. Given a first-countable t.d.l.c. group $G$ and a commensurated compact subgroup $K$ of $G$, then there is a finite index closed subgroup of $K$ that is locally normal in $G$. In particular, $G$ satisfies (S2) if and only if $G$ has no nontrivial fixed points in its action on $\mathcal{L N}(G)$.

A simple observation shows that a t.d.1.c. group with property (S2) cannot have many closed normal subgroups:

LEMMA 3.11. Let $G$ be a t.d.l.c. group with property (S2). Then any closed normal subgroup of $G$ is discrete or open.

Proof. Let $N$ be a closed normal subgroup of $G$ and let $U$ be a compact open subgroup of $G$. The conjugation action of $G$ on $N$ is by automorphisms of the locally compact group and therefore commensurates the compact open subgroup $N \cap U$. Then property (S2) implies that $N \cap U$ is either finite or open, thereby yielding the desired conclusion.

In the present context, it is useful to have a notion of 'size' of certain subsets of a t.d.l.c. group. Recall that infinite compact groups are homogeneous Baire spaces, so in particular they are perfect sets and uncountable. Moreover, a closed subgroup of a compact group that is not open has an empty interior, and therefore has uncountably many cosets.

DEFINITION 3.12. Let $G$ be a t.d.l.c. group and let $K$ be a subgroup of $G$ such that $\mathrm{N}_{G}(K)$ is open. Say a subset $X$ of $G$ is $K$-meagre if $X$ is contained in the union of countably many left cosets of $K$ and $K$-large if it contains a coset of a subgroup of $K$ of finite index. Say $K$ is the local size of $X$ (or $X$ is $K$-sized) if $X$ is $K$-meagre and $K$-large.

The notions of $K$-meagre, $K$-large and $K$-sized are determined by the commensurability class of $K$, so given $\alpha \in \mathcal{L N}(G)$, we can define $\alpha$-meagre, $\alpha$-large and $\alpha$-sized to mean $K$-meagre, $K$-large and $K$-sized respectively, where $K$ is some (any) compact representative of $\alpha$. Say $X$ is locally sized if $X$ has local size $\alpha$ for some $\alpha \in \mathcal{L N}(G)$.

We first make an easy observation that will be used without further comment. (See also [49, Lemma 3.5].) 
LEMMA 3.13. Let $G$ be a group, let $H$ be a commensurated subgroup of $G$ and let $g \in G$. Then $\mathrm{Hg}$ is the union of finitely many left cosets of the finite index subgroup $H \cap g^{-1} H g$ of $H$. Similarly $g H$ is the union of finitely many right cosets of the finite index subgroup $\mathrm{H}_{\mathrm{g} \mathrm{Hg}}^{-1}$ of $\mathrm{H}$.

LEMMA 3.14. Let $G$ be a t.d.l.c.s.c. group.

(i) Let $X$ be a locally sized subset of $G$. Then $X$ is $\alpha$-sized for exactly one $\alpha \in$ $\mathcal{L} \mathcal{N}(G)$, which is both the least element of $\mathcal{L N}(G)$ for which $X$ is $\alpha$-meagre, and the greatest element of $\mathcal{L N}(G)$ for which $X$ is $\alpha$-large.

(ii) Let $K$ be a subgroup of $G$ with open normalizer, and suppose that $G=$ $\operatorname{Comm}_{G}(K)$. Then the product of any two $K$-meagre subsets of $G$ is $K$ meagre. Given a (not necessarily closed) $K$-meagre subgroup $H$ of $G$ such that $\mathrm{N}_{G}(H)$ is open, then $H$ is contained in a $K$-meagre normal subgroup of $G$.

(iii) Let $\alpha \in \mathcal{L N}(G)$. Then the following are equivalent:

(a) $G=\operatorname{Stab}_{G}(\alpha)$;

(b) $G$ has an $\alpha$-sized normal subgroup;

(c) Every $\alpha$-meagre subgroup of $G$ whose normalizer is open is contained in an $\alpha$-sized normal subgroup of $G$.

Proof. (i) Let $\beta, \gamma \in \mathcal{L} \mathcal{N}(G)$ and suppose that $X$ is $\beta$-large and $\gamma$-meagre. Then, since these properties are invariant under left translation, it may be supposed that $X$ contains a representative, $B$, of $\beta$ and $B$ is $\gamma$-meagre because $X$ is. Hence $B$ is the union of countably many left cosets of $B \cap C$, where $C$ is any representative of $\gamma$. Since $B$ is a Baire space, it follows that some (and hence all) left cosets of $B \cap C$ are open in $B$, and then, since $B$ is compact, that $|B: B \cap C|$ is finite. Hence $\beta \leqslant \gamma$ and the local size $\alpha$ of $X$ (if it exists) must be both the least element of $\mathcal{L N}(G)$ for which $X$ is $\alpha$-meagre and the greatest element of $\mathcal{L N}(G)$ for which $X$ is $\alpha$-large.

(ii) Let $X$ and $Y$ be $K$-meagre sets. Then $X Y$ is contained in the union of countably many sets of the form $x K y K$ for $x, y \in G$ and in turn, since $K$ is commensurated by $G$, each such set is the union of finitely many left cosets of $K$. Hence $X Y$ is $K$-meagre.

Repeating the argument, we see that the product of any finite set of $K$-meagre sets is $K$-meagre. Now let $H$ be a $K$-meagre subgroup of $G$ such that $\mathrm{N}_{G}(H)$ is open. By second-countability, there are only countably many cosets of $\mathrm{N}_{G}(H)$ in $G$, hence only countably many $G$-conjugates of $H$. Hence we may make a 
countable list $H_{1}, H_{2}, H_{3}, \ldots$ consisting of $G$-conjugates of $H$ such that every $G$ conjugate appears infinitely often. Conjugates of $K$-meagre sets are $K$-meagre, since $K$ is commensurated by $G$. In particular, we see that $H_{i}$ is $K$-meagre for all $i$. Now notice that the set

$$
L=\bigcup_{i \in \mathbf{N}}\left(H_{1} H_{2} \ldots H_{i}\right)
$$

is a normal subgroup of $G$ containing $H$ and by construction $L$ is $K$-meagre.

(iii) It is clear that (c) implies (b) because $\alpha$ has a representative with open normalizer and every representative of $\alpha$ is $\alpha$-meagre; (b) implies (a) by part (i) because the $\alpha$-sized normal subgroup is also $g . \alpha$-sized for every $g \in G$; and (a) implies (c) by applying part (ii) with $H$ any representative of $\alpha$.

Proof of Theorem 3.9. Suppose $G$ has the given properties. Let $H$ be a nontrivial normal subgroup of $G$. Then $H$ contains a nontrivial commensurated compact locally normal subgroup $L$ of $G$ by property (S1); by property (S2), $L$ and hence $H$ is open, so $H=G$ since $G$ has no proper open normal subgroups by property (S0).

Conversely, suppose $G$ is abstractly simple. Then the only compact normal subgroup of $G$ is the trivial subgroup, so that $G$ is second-countable by Lemma 2.2(iii). Certainly, $G$ contains a compact open subgroup $U$; in particular $U$ is infinite, commensurated and locally normal. Since $G$ is the only nontrivial normal subgroup of $G$, it follows that $G$ satisfies (S0) and (S1). Given an infinite commensurated compact subgroup $K$ of $G$, by applying Lemma 3.10, we see that a finite index subgroup of $K$ is open in $U$. Thus to prove both (S2) and (S2'), it suffices to consider an infinite normal subgroup $K$ of $U$ that is commensurated in $G$. In this case, by Lemma 3.14 (ii), there is a normal subgroup $L$ of $G$ that contains $K$, such that $L$ is $K$-meagre. Since $G$ is abstractly simple, it follows that $G=L$, so $G$ is $K$-meagre; in other words, $K$ has countable index in $G$. The Baire Category Theorem then ensures that $\bar{K}$ is open in $G$, proving that $G$ satisfies (S2) and (S2') as required.

Say a locally compact topological group $G$ is unrefinable if $G$ is nondiscrete, and the only locally compact group topology on $G$ that properly refines the given topology is the discrete one. Recall the following from [21]:

LEMMA 3.15 ([21, Theorem IV] and Lemma 2.4). Let $G$ be a first-countable t.d.l.c. group. Then there is a natural bijection between the elements of $\mathcal{L N}(G)$ that are fixed by the action of $G$, and refinements of the topology of $G$ that are locally compact and compatible with the group structure. 
It is clear that for any t.d.l.c. group $G$, if $G$ is unrefinable then $G$ has (S2). By Lemma 3.15, the converse holds for t.d.l.c.s.c. groups. Theorem 3.9 implies that this applies in particular to abstractly simple t.d.l.c.s.c. groups.

COROLlaRY 3.16. Let $G$ be a nondiscrete t.d.l.c.s.c. group. Then $G$ is unrefinable if and only if $G$ has property (S2). In particular, every abstractly simple t.d.l.c.s.c. group is unrefinable.

\section{Some local properties of compactly generated t.d.l.c. groups}

4.1. Cayley-Abels graphs. We recall the now classical construction, due to Abels, of a family of connected locally finite graphs associated with any compactly generated t.d.l.c. group. (See [2, Beispiel 5.2], or [42, Section 11] for more details.)

Proposition 4.1. Let $G$ be a compactly generated t.d.l.c. group, let $U$ be a compact open subgroup of $G$, let A be a compact symmetric subset of $G$ such that $G=\langle U, A\rangle$ and let $D$ be a dense symmetric subset of $G$.

(i) There exists a finite symmetric subset $B$ of $G$ such that $B \subseteq D$ and

$$
B U=U B=U B U=U A U .
$$

(ii) For any subset $B$ satisfying part (i), then $G=\langle B\rangle U$ and the coset space $G / U$ carries the structure of a locally finite connected graph, invariant under the natural $G$-action, where $g U$ is adjacent to $h U$ if and only if $g U \neq h U$ and $U g^{-1} h U \subseteq U B U$.

Proof. (i) Since $A U$ is compact, it is contained in a union of finitely many right cosets of $U$, so $U A U$ is a union of finitely many right cosets of $U$; say $U A U=$ $\bigcup_{b \in B_{1}} U b$ for $B_{1}$ finite. Similarly, $U A U=\bigcup_{b \in B_{2}} b U$ for $B_{2}$ finite. Since all the right and left cosets of $U$ are open, we are free to choose $B_{1}$ and $B_{2}$ to be subsets of $D$. Now set

$$
B=B_{1} \cup B_{1}^{-1} \cup B_{2} \cup B_{2}^{-1} ;
$$

it is straightforward to verify that $B$ has the required properties.

(ii) It is clear that the given adjacency relation is well defined, and the fact that $B=B^{-1}$ ensures that it is a symmetric relation. Moreover, given $g, h, k \in G$, we see that $U(k g)^{-1}(k h) U=U g^{-1} h U$, so $k g U$ is adjacent to $k h U$ if and only if $g U$ is adjacent to $h U$, in other words, $G$ preserves the graph structure. Since $U B=$ $U A U$ and $G=\langle U, A\rangle$, we have $G=\langle U, B\rangle$; the fact that $G=\langle B\rangle U$ follows 
by repeatedly applying the equation $U B=B U$. Given $h \in\langle B\rangle$, there is clearly a path from $U$ to $h U$, ensuring that the graph defined on $G / U$ is connected.

Since $G$ acts transitively on $G / U$ and preserves adjacency, to see that the graph is locally finite it is enough to see that the trivial coset $U$ has finitely many neighbours. Indeed, $U$ is adjacent to $g U$ for $g \in G \backslash U$ if and only if $g U \subseteq U B U$. But $U B U=B U$ is the union of finitely many left cosets of $U$, so there are only finitely many possibilities for $g U$.

The graph provided by Proposition 4.1 will be called a Cayley-Abels graph associated with $(G, U)$. We emphasize that its edge set depends on the set $U B U$. All Cayley-Abels graphs of $G$ are quasi-isometric.

The next lemma in turn uses this information to strengthen considerably the observation that, if $G$ is nondiscrete, compactly generated and topologically simple and $U$ is a compact open subgroup of $G$, then finitely many conjugates of $U$ suffice to generate $G$.

LEMMA 4.2. Let $G$ be a compactly generated t.d.l.c. group and let $L$ be a (possibly nonclosed) locally normal subgroup. Suppose the abstract normal closure $D=\langle\langle L\rangle\rangle$ is dense in $G$. Then there are $g_{1}, \ldots, g_{n}$ in $D$ such that $D=\left\langle L \cup g_{1} L g_{1}^{-1} \cup \cdots \cup g_{n} L g_{n}^{-1}\right\rangle$.

Proof. Let $U$ be a compact open subgroup that normalizes $L$. Since $D$ is dense in $G$, by Proposition 4.1 we can find a finite set $\Sigma \subset D$ such that $G=\langle\Sigma\rangle U$. Since $U$ normalizes $L$, the conjugation action of $\langle\Sigma\rangle$ is thus transitive on the $G$-conjugacy class of $L$. Hence the subgroup $\langle\Sigma \cup L\rangle$ of $D$ contains the entire conjugacy class of $L$, which, since $D$ is generated by that conjugacy class, implies that $D=\langle\Sigma \cup L\rangle$. Since it belongs to $D$, each element of $\Sigma$ is a product of a finite number of conjugates of elements of $L$. We may therefore choose $g_{1}, \ldots$, $g_{n} \in\langle\Sigma\rangle$ such that $\Sigma \subset\left\langle L \cup g_{1} L g_{1}^{-1} \cup \cdots \cup g_{n} L g_{n}^{-1}\right\rangle$, and the claim follows.

4.2. Quasicentralizers of commensurated subgroups. We now prove two related propositions. The second may be viewed as a generalization of [5, Theorems 4.8 and 4.9].

Proposition 4.3. Let $G$ be a t.d.l.c.s.c. group, let $N$ be a minimal closed normal subgroup of $G$ and let $H$ be an infinite closed subgroup of $N$ such that $H$ is commensurated by $G$ and $\mathrm{QZ}(H)=\{1\}$. Then the quasicentralizer $\mathrm{QC}_{G}(H)$ is a closed normal subgroup of $G$ that intersects $N$ trivially. In particular, if $G$ is monolithic and $\mathrm{QZ}(N)=\{1\}$, then $\mathrm{QC}_{G}(H)=\mathrm{QC}_{G}(N)=\{1\}$. 
Proof. The hypothesis that $\mathrm{QZ}(H)=\{1\}$ ensures that $H$ is nondiscrete. By Lemma 2.4, there exists a compact open subgroup $U$ of $G$ such that $H \cap U$ is locally normal in $G$. Since $\mathrm{QZ}(H \cap U)=\mathrm{QZ}(H)=\{1\}$, it follows from [21, Lemma 3.8(i)] that

$$
\mathrm{QC}_{G}(H \cap U) \cap \mathrm{N}_{G}(H \cap U)=\mathrm{C}_{G}(H \cap U) .
$$

In particular, since $\mathrm{N}_{G}(H \cap U)$ is open in $G$ and $\mathrm{C}_{G}(H \cap U)$ is closed, we deduce that $\mathrm{QC}_{G}(H \cap U)=\mathrm{QC}_{G}(H)$ is closed in $G$. Making the abbreviation $Q=\mathrm{QC}_{G}(H) \cap N$, we deduce that $Q$ is closed. Moreover, $\mathrm{QC}_{G}(H)$ is normal in $G$, since $G$ commensurates $H$, hence also $H \cap U$. Therefore, if $Q$ is nontrivial, we must have $Q=N$ by minimality of $N$. Recalling that $H \leqslant N$ by hypothesis, we obtain $H \leqslant Q \leqslant \mathrm{QC}_{G}(H)$, so $H=\mathrm{QC}_{H}(H)=\mathrm{QZ}(H)$, which is absurd as $H$ is infinite and $\mathrm{QZ}(H)=\{1\}$. From this contradiction we deduce that $\mathrm{QC}_{G}(H) \cap N=\{1\}$. The final conclusion is clear.

Proposition 4.4. Let $G \in \mathscr{S}$. Then every infinite closed subgroup that is commensurated by $G$ has a trivial quasicentralizer. In particular, $\mathrm{QZ}(G)=\{1\}$.

Proof. Let $H$ be an infinite closed subgroup that is commensurated by $G$. For any compact open subgroup $W$ of $G$, the compact group $H \cap W$ is then also commensurated by $G$, and moreover we have $\mathrm{QC}_{G}(H)=\mathrm{QC}_{G}(H \cap W)$. Therefore, it suffices to prove the proposition in case $H$ is compact, which we assume henceforth. Invoking Lemma 2.4 as in Proposition 4.3, we may moreover assume that $H$ is locally normal.

Let $Q=\mathrm{QC}_{G}(H)$. We see that $Q$ is normal in $G$, since $G$ fixes [H]. Suppose that $Q \neq\{1\}$. Then $Q$ is dense in $G$, since $G$ is topologically simple.

Let $U$ be a compact open subgroup of $G$ that normalizes $H$. Then $G$ is generated by $U X$ where $X$ is a finite subset of $G$ which, by Proposition 4.1, may be chosen to be a subset of $Q$. Since $X$ is finite, there is an open subgroup $K$ of finite index in $H$ that is centralized by $X$. Choose $V$ open and normal in $U$ such that $V \cap H \leqslant K$. Then $L:=V \cap H$ is normalized by all elements of $U \cup X$, and is a nontrivial compact normal subgroup of $G$. Since $G$ is topologically simple, we conclude that $G$ is compact, hence profinite. But the only topologically simple profinite groups are the finite simple groups, which are discrete. As $G$ is assumed nondiscrete, we have a contradiction. Thus $Q$ must be trivial as required. The final conclusion follows by considering $\mathrm{QC}_{G}(U)$ where $U$ is a compact open subgroup of $G$, since $\mathrm{QZ}(G)=\mathrm{QC}_{G}(U)$.

4.3. The local prime content. Cayley-Abels graphs can be applied to obtain a restriction on the primes involved in the compact open subgroups of a compactly generated t.d.l.c. group. 
DEFINITION 4.5. Let $G$ be a t.d.l.c. group. The local prime content $\eta(G)$ of $G$ is the set of primes $p$ for which $G$ contains an infinite pro- $p$ subgroup.

Proposition 4.6. Let $G$ be a nondiscrete compactly generated t.d.l.c. group. Then $G$ has a compact normal subgroup $K$, which can be chosen to be a subgroup of any given open neighbourhood of the identity, such that for every compact open subgroup $U$ of $G$, the composition factors of $U K / K$ are of bounded order. In particular, the set $\eta=\eta(G / K)$ is finite and $U K / K$ is virtually pro- $\eta$ for every compact open subgroup $U$.

Proof. By Lemma 2.2 we may assume that $G$ is second-countable. Let $O$ be an open neighbourhood of the identity in $G$. Then by van Dantzig's theorem, $O$ contains a compact open subgroup $V$ of $G$. Let $\Gamma$ be a Cayley-Abels graph associated to $(G, V)$. Let $K$ be the kernel of the action of $G$ on $\Gamma$. Then $K=$ $\bigcap_{g \in G} g V g^{-1}$, so $K$ is compact and $K \subseteq O$. From now on we may assume $K=$ $\{1\}$. Vertex transitivity of the action of $G$ on $\Gamma$ implies that all vertices have the same valency, $d$.

Let $U$ be a compact open subgroup of $G$ and consider the action of $U$ on $\Gamma$. Let $U_{0}=U \cap V$ and for all $n>0$, let $U_{n}$ be the pointwise stabilizer of the $n$ sphere around the base vertex $v_{0}=V$ in $U_{0}$. Thus the collection $\left\{U_{n}\right\}_{n \geqslant 0}$ is a descending chain of open normal subgroups of $U_{0}$ with trivial intersection. For each $n \geqslant 0$, the finite group $U_{n} / U_{n+1}$ fixes pointwise the $n$-sphere around $v_{0}$ and acts faithfully on the $n+1$-sphere. Since $\Gamma$ is $d$-regular, the orbits of $U_{n} / U_{n+1}$ on the $n+1$-sphere have size at most $d$, so every composition factor of $U_{1}$ embeds in $\operatorname{Sym}(d)^{m}$ for some $m$ and thus has order dividing $d !$. In particular, $U_{1}$ is a pro- $\pi$ group where $\pi$ is the set of primes less than $d+1$. Hence $\eta \subseteq \pi$ and is finite. For each of the finitely many primes $p \in \pi \backslash \eta$, the $p$-Sylow subgroup $P_{p}$ of $U_{1}$ is finite, so there exists an open normal subgroup $V_{p}$ of $U_{1}$ that intersects $P_{p}$ trivially. Sylow's theorem then implies $V_{p}$ is pro- $p^{\prime}$, and by intersecting finitely many such open subgroups of $U_{1}$, we obtain an open pro- $\eta$ subgroup $V$, which is then also of finite index in $U$. Hence $U$ is virtually pro- $\eta$.

In order to obtain stronger results of this kind, we recall some vocabulary from finite group theory, adapted to the profinite setting.

DEFINITION 4.7. Let $G$ be a profinite group. For a set of primes $\pi$, the $\pi$-core $O_{\pi}(G)$ is the largest normal pro- $\pi$ subgroup of $G$, and the $\pi$-residual $O^{\pi}(G)$ is the smallest closed normal subgroup of $G$ such that $G / O^{\pi}(G)$ is pro- $\pi$. The prosoluble core $O_{\infty}(G)$ is the largest normal prosoluble subgroup of $G$, while the prosoluble residual $O^{\infty}(G)$ is the smallest closed normal subgroup of $G$ such that $G / O^{\infty}(G)$ is prosoluble. 
LEMMA 4.8. Let $G$ be a t.d.l.c. group, let $U$ and $V$ be compact open subgroups of $G$ and let $\pi$ be a set of primes. Then $O_{\pi}(U)$ is commensurate with $O_{\pi}(V)$; similarly, $O_{\infty}(U)$ is commensurate with $O_{\infty}(V)$. Consequently

$$
G=\operatorname{Comm}_{G}\left(O_{\pi}(U)\right)=\operatorname{Comm}_{G}\left(O_{\infty}(U)\right) .
$$

Proof. Let $W$ be an open subgroup of $U \cap V$ that is normal in $V$. Then $O_{\pi}(U) \cap W$ is pro- $\pi$ and normal in $W$, so contained in $O_{\pi}(W)$. In turn $O_{\pi}(W)$ is pro- $\pi$ and normal in $V$, so $O_{\pi}(W) \leqslant O_{\pi}(V)$. Hence $O_{\pi}(V)$ contains an open subgroup of $O_{\pi}(U)$ and vice versa by symmetry. Hence $O_{\pi}(U)$ is commensurate with $O_{\pi}(V)$.

The proof of the analogous statement about the prosoluble residual is the same.

Before proving the main result of this section, Theorem 4.13, we recall an observation of Wielandt (originally made in the context of finite groups) about the normalizers of $\pi$-residuals and prosoluble residuals (compare [14, Lemma 2.2.3]).

LEMMA 4.9. Let $G$ be a profinite group and let $S$ be a subnormal subgroup of $G$. Then for each set of primes $\pi$, the group $O_{\pi}(G)$ normalizes $O^{\pi}(S)$. Similarly $O_{\infty}(G)$ normalizes $O^{\infty}(S)$.

Proof. Let $*$ stand for either a set of primes $\pi$, or $\infty$, and let $\mathcal{C}$ be the class of pro$\pi$ groups or the class of pro-(finite soluble) groups respectively. Let $H=O_{*}(G) S$. Since

$$
S /\left(S \cap O^{*}(H)\right) \cong S O^{*}(H) / O^{*}(H)
$$

and $\mathcal{C}$ is closed under taking closed subgroups, we see that $S /\left(S \cap O^{*}(H)\right)$ is in $\mathcal{C}$. Hence $O^{*}(S) \leqslant O^{*}(H)$. We claim that in fact $O^{*}(S)=O^{*}(H)$.

Since $S$ is subnormal in $G$, it is also subnormal in $H$, and there is a series

$$
S=H_{0} \triangleleft H_{1} \triangleleft \cdots \triangleleft H_{n}=H .
$$

In order to prove the claim, observe first that, since $H=O_{*}(G) S$ and since $S \leqslant H_{i}$ for all $i$, we have $H_{i}=H_{i} \cap O_{*}(G) S=O_{*}\left(H_{i}\right) S$ for all $i$. We now proceed to prove the claim by induction on $n$. By the inductive hypothesis, we may assume $O^{*}(S)=O^{*}\left(H_{n-1}\right)$. Now $O^{*}\left(H_{n-1}\right)$ is characteristic in $H_{n-1}$, hence normal in $H_{n}$. Thus we have a quotient $H_{n} / O^{*}\left(H_{n-1}\right)$ of $H_{n}$, which is an extension of $A=H_{n} / H_{n-1}$ by $B=H_{n-1} / O^{*}\left(H_{n-1}\right)$. Since $H_{n}=O_{*}\left(H_{n}\right) S$ and $S \leqslant H_{n-1}$, we see that $A$ is isomorphic to a quotient of $O_{*}\left(H_{n}\right)$, which is in $\mathcal{C}$, so $A \in \mathcal{C}$, while $B \in \mathcal{C}$ by the definition of $O^{*}\left(H_{n-1}\right)$. Since $\mathcal{C}$ is closed under extensions within 
the class of profinite groups, we have $H_{n} / O^{*}\left(H_{n-1}\right) \in \mathcal{C}$, so $O^{*}\left(H_{n}\right) \leqslant O^{*}\left(H_{n-1}\right)$ and hence $O^{*}\left(H_{n}\right)=O^{*}\left(H_{n-1}\right)=O^{*}(S)$.

Since $H_{n}=H$ and since $O^{*}(H)$ is normal in $H$ and $O_{*}(G)$ is a subgroup of $H$, it follows that $O_{*}(G)$ normalizes $O^{*}(S)$.

It is a fact well known and frequently used that, if $H$ and $K$ are subgroups of $G$ that normalize each other and have trivial intersection, then $H$ centralizes $K$. The following lemma, recalled from [21], is the local version of this fact.

LEMMA 4.10 [21, Lemma 3.9]. Let $G$ be a t.d.l.c. group and let $H$ and $K$ be closed subgroups of $G$ such that $\mathrm{QZ}(H)=\{1\}$ and such that $|H \cap K|$, $\left|H: \mathrm{N}_{H}(K)\right|$ and $\left|K: \mathrm{N}_{K}(H)\right|$ are all finite. Then $H$ centralizes a finite index open subgroup of $K$.

COROLlaRY 4.11. Let $G$ be a t.d.l.c. group and let $H$ and $K$ be nontrivial compact locally normal subgroups of $G$ such that $\mathrm{QZ}(H)=\mathrm{QC}_{G}(K)=\{1\}$. Then $H \cap K$ is infinite.

Proof. Since $H$ and $K$ are locally normal, we see that $\left|H: \mathrm{N}_{H}(K)\right|$ and $\left|K: \mathrm{N}_{K}(H)\right|$ are finite. Suppose also that $H \cap K$ is finite. By Lemma 4.10, $H$ centralizes an open subgroup of $K$, that is, $H \leqslant \mathrm{QC}_{G}(K)$. Since $\mathrm{QC}_{G}(K)$ is trivial but $H$ is not, we have a contradiction.

Theorems 4.13 and 4.14 are the culmination of this section. Before proceeding to them, we record the following subsidiary fact separately.

Proposition 4.12. Let $G$ be a nontrivial compactly generated t.d.l.c. group. Suppose that $G$ has trivial quasicentre, no nontrivial compact normal subgroups and no nontrivial compact abelian locally normal subgroups. Let $H$ be a nontrivial closed locally normal subgroup of $G$. Then there is a minimal closed normal subgroup $M$ of $G$ such that $H \cap M$ is nondiscrete.

Proof. By Proposition 2.12, every nontrivial closed normal subgroup of $G$ contains a minimal one, and the set $\left\{M_{1}, \ldots, M_{n}\right\}$ of minimal closed normal subgroups of $G$ is finite. Proposition 2.7 ensures that $\mathrm{QC}_{G}\left(M_{i}\right)=\mathrm{C}_{G}\left(M_{i}\right)$ for all $i$. The intersection $\bigcap_{i=1}^{n} \mathrm{QC}_{G}\left(M_{i}\right)$ is thus a closed normal subgroup of $G$. This intersection centralizes, and therefore does not contain, each of the nonabelian subgroups $M_{i}$. Hence $\bigcap_{i=1}^{n} \mathrm{QC}_{G}\left(M_{i}\right)$ is trivial.

By Proposition 2.7 we have $\mathrm{QZ}(H)=\{1\}$, so $H$ is nondiscrete. Since $\bigcap_{i=1}^{n} \mathrm{QC}_{G}\left(M_{i}\right)=\{1\}$, there is $i \in\{1, \ldots, n\}$ such that $\mathrm{QC}_{G}\left(M_{i}\right)$ does not contain an open subgroup of $H$. In particular, no open subgroup of $H$ centralizes any open 
subgroup of $M_{i}$. Given a compact open subgroup $U$ of $G$, then $H^{\prime}=H \cap U$ and $M_{i}^{\prime}=M_{i} \cap U$ are both compact and locally normal in $G$. It then follows that the indices $\left|H^{\prime}: \mathrm{N}_{H^{\prime}}\left(M_{i}^{\prime}\right)\right|$ and $\left|M_{i}^{\prime}: \mathrm{N}_{M_{i}^{\prime}}(H)\right|$ are both finite, while QZ $\left(H^{\prime}\right)=\{1\}$. By Lemma 4.10 it follows that $H^{\prime} \cap M_{i}^{\prime}$ is an infinite compact group, so $H \cap M_{i}$ is nondiscrete.

THEOREM 4.13. Let $G$ be a nontrivial compactly generated t.d.l.c. group. Suppose that $G$ has trivial quasicentre, no nontrivial compact normal subgroups and no nontrivial compact abelian locally normal subgroups. Let $\left\{M_{1}, \ldots, M_{n}\right\}$ be the set of minimal nontrivial closed normal subgroups of $G$ (which is indeed finite by Proposition 2.12) and let $\eta_{i}=\eta\left(M_{i}\right)$ for $1 \leqslant i \leqslant n$.

Then there are nonempty subsets $\eta_{i}^{*} \subseteq \eta_{i}$ for $1 \leqslant i \leqslant n$ such that given any nontrivial compact locally normal subgroup $H$ of $G$ and any set of primes $\xi$, $O_{\xi}(H)$ is nontrivial if and only if there is some $i \in\{1, \ldots, n\}$ such that $\xi \supseteq \eta_{i}^{*}$ and $H \cap M_{i}>1$. Moreover, if $\xi=\bigcup_{i=1}^{n} \eta_{i}^{*}$, then $O_{\xi}(H)$ is infinite for all such $H$.

Proof. Recall that by Proposition 2.7, every closed locally normal subgroup of $G$ has trivial quasicentre.

Fix $i \in\{1, \ldots, n\}$. Let $\mathcal{D}_{i}$ be the collection of those sets, $\xi$, of primes such that for some compact open subgroup $U$ of $G$, the $\xi$-core $O_{\xi}\left(U \cap M_{i}\right)$ is infinite. Notice that if $\xi \in \mathcal{D}_{i}$, then in fact $O_{\xi}\left(U \cap M_{i}\right)$ is infinite for every compact open subgroup $U$. Let $U$ be a compact open subgroup of $G$ and let $\xi_{1}, \xi_{2} \in \mathcal{D}_{i}$. Then the compact subgroups $O_{\xi_{1}}\left(U \cap M_{i}\right)$ and $O_{\xi_{2}}\left(U \cap M_{i}\right)$ are locally normal in $G$, and thus both have trivial quasicentre, and $O_{\xi_{1}}\left(U \cap M_{i}\right)$ and $O_{\xi_{2}}\left(U \cap M_{i}\right)$ normalize each other. At the same time, $O_{\xi_{1}}\left(U \cap M_{i}\right)$ and $O_{\xi_{2}}\left(U \cap M_{i}\right)$ both have a trivial quasicentralizer in $M_{i}$ by Proposition 4.3 because, as Lemma 4.8 shows, they are commensurated in $G$. By applying Corollary 4.11 to the subgroups $O_{\xi_{1}}\left(U \cap M_{i}\right)$ and $O_{\xi_{2}}\left(U \cap M_{i}\right)$ of the group $M_{i}$, it follows that

$$
O_{\xi_{1}}\left(U \cap M_{i}\right) \cap O_{\xi_{2}}\left(U \cap M_{i}\right)=O_{\xi_{1} \cap \xi_{2}}\left(U \cap M_{i}\right)
$$

is infinite. This shows that $\mathcal{D}_{i}$ is closed under finite intersections. Moreover, $\mathcal{D}_{i}$ contains $\eta(G)$, which is a finite set by Proposition 4.6. Thus the poset $\mathcal{D}_{i}$ has a smallest element $\eta_{i}^{*} \subseteq \eta(G)$, which is necessarily nonempty by the definition of $\mathcal{D}_{i}$.

Now let $H$ be a nontrivial compact locally normal subgroup of $G$, let $\xi$ be a set of primes and fix a compact open subgroup $U<G$ containing $H$ as a normal subgroup.

Suppose there is some $i$ such that $i \in\{1, \ldots, n\}$ such that $\xi \supseteq \eta_{i}^{*}$ and $H \cap M_{i}>$ 1. Then $\mathrm{QC}_{M_{i}}\left(O_{\xi}\left(U \cap M_{i}\right)\right)$ is trivial, as above, and hence $O_{\xi}\left(U \cap M_{i}\right) \cap H$ is infinite by Corollary 4.11. Since $O_{\xi}\left(U \cap M_{i}\right) \cap H$ is a normal pro- $\xi$ subgroup 
of $H$, it is contained in $O_{\xi}(H)$ and we have thus shown that $O_{\xi}(H)$ is infinite. Conversely, suppose that $O_{\xi}(H)$ is nontrivial. Then by Proposition 4.12, there exists $i \in\{1, \ldots, n\}$ such that $O_{\xi}(H) \cap M_{i}$ is infinite, so in particular $H \cap M_{i}>1$. At the same time, $O_{\xi}(H)$ is a characteristic subgroup of $H$, and hence a normal subgroup of $U$, so $O_{\xi}(H) \cap M_{i}$ is an infinite normal pro- $\xi$ subgroup of $U \cap M_{i}$. Thus $O_{\xi}\left(U \cap M_{i}\right)$ is infinite, in other words $\xi \in \mathcal{D}_{i}$, ensuring that $\xi \supseteq \eta_{i}^{*}$. We have now shown that $O_{\xi}(H)$ is nontrivial if and only if there is some $i \in\{1, \ldots, n\}$ such that $\xi \supseteq \eta_{i}^{*}$ and $H \cap M_{i}>1$.

Finally, suppose that $\xi=\bigcup_{i=1}^{n} \eta_{i}^{*}$. By Proposition 4.12 there exists $i \in\{1, \ldots$, $n\}$ such that $H \cap M_{i}$ is nondiscrete. Since $\xi \supseteq \eta_{i}^{*}$, the previous paragraph implies $O_{\xi}(H)$ is infinite, as required.

We can prove a stronger result for locally C-stable t.d.l.c. groups that are in $\mathscr{S}$. In fact it will be seen later, in Theorem 5.3, that every group in $\mathscr{S}$ is locally C-stable, so the following theorem applies to all groups in $\mathscr{S}$.

THEOREM 4.14. Let $G$ be a nontrivial compactly generated, topologically simple, locally C-stable t.d.l.c. group. Then the following hold.

(i) Every nontrivial compact locally normal subgroup $H \leqslant G$ is virtually pro$\eta(G)$ and, if $H$ is virtually pro- $\xi$ for some set of prime numbers $\xi$, then $\xi \supseteq$ $\eta(G)$.

(ii) If $G$ contains a nontrivial prosoluble locally normal subgroup, then every compact open $U \leqslant G$ is virtually prosoluble.

Proof. If $G$ is discrete, all assertions are trivial. We assume henceforth that $G$ is nondiscrete. We then note that $G$ satisfies the hypotheses of Theorem 4.13: we have $\mathrm{QZ}(G)=\{1\}$ by Proposition 4.4 and the absence of nontrivial compact abelian locally normal subgroups is ensured by Proposition 2.7.

For (i), by Theorem 4.13 there is $\eta^{*} \subseteq \eta(G)$ such that, given any nontrivial compact locally normal subgroup $H$ of $G$ and any set of primes $\xi, O_{\xi}(H)$ is nontrivial if and only if $\xi \supseteq \eta^{*}$. We have to show that $\eta^{*}=\eta(G)$; it suffices to show that some compact open subgroup $U$ of $G$ is virtually pro- $\eta^{*}$. Set $L=$ $O_{\eta^{*}}(U)$.

For (ii), we take $U$ to be a compact open subgroup and set $L=O_{\infty}(U)$; in this case we may assume that $L$ is nontrivial, and our aim is to show that $U$ is virtually prosoluble.

In both cases $L$ is a nontrivial compact locally normal subgroup. From this point onwards the proofs of (i) and (ii) are similar: let $\star$ stand for $\eta^{*}$ for the proof of (i), and $\infty$ for the proof of (ii). 
Lemma 4.2 implies that the normal closure of $L$ is generated by a finite set of conjugates of $L$. Let thus $g_{1}, \ldots, g_{n} \in G$ be such that $D=\left\langle L \cup g_{1} L g_{1}^{-1} \cup\right.$ $\left.\cdots g_{n} L g_{n}^{-1}\right\rangle$ is dense and normal in $G$. Set $g_{0}=1$ and $U_{i}=g_{i} U g_{i}^{-1}$ for all $i=0$, $\ldots, n$. Let also $V_{0}$ be an open normal subgroup of $U_{0}$ contained in $\bigcap_{i=0}^{n} U_{i}$. For all $i>0$, define inductively a group $V_{i}$ as the normal core of $V_{i-1}$ in the group $U_{i}$. Thus $V_{i} \triangleleft U_{i}$ and for all $i \in\{0,1, \ldots, n\}$, we get a subnormal chain

$$
V_{n} \triangleleft V_{n-1} \triangleleft \cdots \triangleleft V_{i} \triangleleft U_{i} .
$$

From Lemma 4.9, we infer that $O^{\star}\left(V_{n}\right)$ is normalized by $O_{\star}\left(U_{i}\right)$ for all $i=$ $0, \ldots, n$. Notice that $O_{\star}\left(U_{i}\right)=g_{i} L g_{i}^{-1}$. We deduce that $O^{\star}\left(V_{n}\right)$ is normalized by $D$. Since $O^{\star}\left(V_{n}\right)$ is compact, its normalizer in $G$ is closed. Therefore, $O^{\star}\left(V_{n}\right)$ is normal in $G$ since $D$ is dense.

Topological simplicity of $G$ then implies that $O^{\star}\left(V_{n}\right)$ is trivial. In other words $V_{n}$ is a pro- $\eta^{*}$ group for part (i), respectively a prosoluble group for part (ii). Since $V_{n}$ is open in $U$ by construction, this proves that $U$ is virtually pro- $\eta^{*}$ or prosoluble respectively, as required.

\section{Properties of the structure lattice}

5.1. The orbit join property. Recall that $\mathscr{S}$ is the class of nondiscrete compactly generated topologically simple t.d.l.c. groups. We have seen (Theorem 3.9) that if $G \in \mathscr{S}$ is abstractly simple, then $G$ has no nontrivial fixed points in $\mathcal{L N}(G)$, and thus all $G$-orbits on $\mathcal{L N}(G) \backslash\{0, \infty\}$ are infinite. However, it turns out that the orbits of $G$ on $\mathcal{L N}(G)$ are still 'compact' in a certain sense: each orbit has a least upper bound, which is the join of finitely many elements of the orbit. In the case that $G$ satisfies (S2), it follows that every compact locally normal subgroup $H$ of $G$ is relatively large, in the sense that there exists a finite set of $G$-conjugates of $H$ whose product has a nonempty interior. Even without assuming (S2), we see that in the conjugation action of $G$ on $\mathcal{L} \mathcal{N}(G)$, the lattice $\mathcal{L N}(G)^{G}$ of fixed points plays an important role. We work here in the more general setting of Hecke pairs $(G, U)$ and, in this setting, a subgroup $K \leqslant G$ is bounded if it is contained in a finite number of $U$-cosets and is locally normal if $\mathrm{N}_{G}(K) \cap U$ has finite index in $U$.

LEMma 5.1. Let $(G, U)$ be a Hecke pair such that $G$ is generated by finitely many cosets of $U$. Let $\kappa$ be a set of bounded locally normal subgroups which is invariant under the $U$-action by conjugation, and such that $G=\langle U, \kappa\rangle$. Then there is a finite set $\left\{K_{1}, \ldots, K_{n}\right\} \subseteq \kappa$ which is a union of $U$-orbits under the conjugation action, such that $G=\left\langle U, K_{1}, \ldots, K_{n}\right\rangle$. Moreover, setting $V:=$ $\bigcap_{i=1}^{n} \mathrm{~N}_{U}\left(K_{i}\right)$ and $L_{i}:=V \cap K_{i}$ for all $i$, we have the following: 
(i) for each $i, L_{i}$ is a subgroup of finite index in $K_{i}$;

(ii) $L_{i}$ normalizes both $K_{j}$ and $L_{j}$ for all pairs $(i, j)$; and

(iii) $L=\prod_{i=1}^{n} L_{i}$ is a normal subgroup of $U$ that is commensurated by $G$.

Proof. We have $G=\langle U, X\rangle$ for some finite set $X$ where, since $G=\langle U, \kappa\rangle$, we can choose $X=\left\{x_{1}, \ldots, x_{n}\right\}$ and $\left\{K_{1}, \ldots, K_{n}\right\} \subseteq \kappa$ such that $x_{i} \in K_{i}$. Then $G=\left\langle U, K_{1}, \ldots, K_{n}\right\rangle$. Since every element of $\kappa$ is locally normal, it has only finitely many conjugates under the action of $U$, and so we may assume that $\left\{K_{1}\right.$, $\left.\ldots, K_{n}\right\}$ is a union of $U$-conjugacy classes by enlarging $n$. Set $V=\bigcap_{i=1}^{n} \mathrm{~N}_{U}\left(K_{i}\right)$ and let $L_{i}=V \cap K_{i}$. Clearly $\left|K_{i}: L_{i}\right|$ is finite, and $L_{i}$ normalizes $K_{j}$ and $L_{j}$ for all pairs $(i, j)$. Furthermore, the conjugation action of $U$ preserves the set $\left\{L_{1}\right.$, $\left.\ldots, L_{n}\right\}$ because $V$ is normal in $U$. Hence $L=\prod_{i=1}^{n} L_{i}$ is a normal subgroup of $U$. Since $L$ is a finite index subgroup of $K_{i} L$ for each $i$, it follows that $K_{i} L$ commensurates $L$ and hence that $\operatorname{Comm}_{G}(L) \geqslant\left\langle U, K_{1} L, \ldots, K_{n} L\right\rangle=G$.

We can now prove Theorem B.

Proof of Theorem B. Every $G \in \mathscr{S}$ is first-countable by Corollary 2.3, so conditions (i) and (ii) are equivalent by Lemma 3.10. The equivalence of (i) and (iii) is a special case of Corollary 3.16. It only remains to show that (i) and (iv) are equivalent.

Suppose (i) holds and let $H$ be a nontrivial compact locally normal subgroup of $G$. By Proposition 4.4, we have $\mathrm{QZ}(G)=\{1\}$, so $H$ must be infinite. Since $G$ is topologically simple, we have $G=\overline{\langle\langle H\rangle}$. Applying Lemma 5.1, we produce a set $\left\{L_{1}, \ldots, L_{d}\right\}$ of infinite subgroups of $G$, such that for all $1 \leqslant i \leqslant d$, there exists $g_{i} \in G$ such that $L_{i} \leqslant g_{i} H g_{i}^{-1}$, and such that $L=\prod_{i=1}^{n} L_{i}$ is a commensurated compact locally normal subgroup of $G$. Since $G$ has (S2), in fact $L$ is an open subgroup of $G$. Since $L \leqslant\left\langle g_{i} H g_{i}^{-1} \mid 1 \leqslant i \leqslant d\right\rangle$, we have $L \leqslant H$, so that $H$ is open. In particular $G=\langle\langle H\rangle\rangle$. Assertion (iv) now follows from Lemma 4.2.

Conversely, suppose (iv) holds and let $H$ be an infinite commensurated compact subgroup of $G$. Then by Lemma 3.10, there is a finite index subgroup $K$ of $H$ that is locally normal in $G$. By (iv), there exist $g_{1}, \ldots, g_{n} \in G$ such that $G$ is generated by $X=\bigcup_{i=1}^{n} g_{i} K g_{i}^{-1}$. Since $H$ is commensurated in $G, K$ is also commensurated, and we see that $X$ is $K$-meagre. Since $X$ generates $G$ it follows by Lemma 3.14 that $G$ is $K$-meagre, and hence $|G: K|$ is countable. The Baire Category Theorem then implies that $K$ is open in $G$, so $H$ is open in $G$. Thus (iv) implies (i).

Lemma 5.1 also leads to a version of Theorem D. 
THEOREM 5.2. Let $G$ be in $\mathscr{S}$. Then, for each $\alpha \in \mathcal{L N}(G)$, there is a unique smallest fixed point, $\alpha^{*} \in \mathcal{L N}(G)^{G}$, that is greater than or equal to $\alpha$. Moreover, there is a finite subset $\left\{g_{1}, \ldots, g_{n}\right\}$ of $G$ such that $\alpha^{*}=\bigvee_{i=1}^{n} g_{i} \alpha$.

Proof. Choose a compact locally normal subgroup $K$ of $G$ such that $[K]=\alpha$ and let $U$ be a compact open subgroup of $G$. Then $G=\langle U, \kappa\rangle$, where $\kappa$ is the $G$-conjugacy class of $K$. Since $\mathrm{N}_{G}(K)$ is open, $U$ acts on $\kappa$ by conjugation with finite orbits, and the compactness of $K$ guarantees that $|M: M \cap U|<\infty$ for all $M \in \kappa$. The conditions of Lemma 5.1 are therefore satisfied and so there is a finite subset $\left\{K_{1}, \ldots, K_{n}\right\} \subseteq \kappa$ and finite index subgroups $L_{i}$ of $K_{i}$ such that $L=\prod_{i=1}^{n} L_{i}$ is a normal subgroup of $U$ that is commensurated by $G$. In the present setting, the subgroups $L_{i}$ provided by Lemma 5.1 are closed and so $L$ is compact. Now $\gamma=[L]$ is fixed by the conjugation action of $G$ on $\mathcal{L N}(G)$ and we can express $\gamma$ as

$$
\gamma=\bigvee_{i=1}^{n}\left[L_{i}\right]=\bigvee_{i=1}^{n}\left[K_{i}\right]=\bigvee_{i=1}^{n} g_{i} \alpha,
$$

where $g_{1}, \ldots, g_{n}$ are elements of $G$ such that $K_{i}=g_{i} K_{i}^{-1}$.

Suppose that $\beta \in \mathcal{L} \mathcal{N}(G)^{G}$ with $\beta \geqslant \alpha$. Then $\beta=g_{i} \beta \geqslant g_{i} \alpha$ for all $i=1$, $\ldots, n$ and so $\beta \geqslant \gamma$. Hence $\gamma$ is the unique minimal element of the set $\{\beta \in$ $\left.\mathcal{L N}(G)^{G} \mid \beta \geqslant \alpha\right\}$.

\subsection{On the nonexistence of virtually abelian locally normal subgroups.} We would like to investigate (topologically) simple t.d.l.c. groups by means of the Boolean algebras defined in [21], as we have better control over their structure than we do for the structure lattice as a whole. Recall that to ensure the centralizer and local decomposition lattices are indeed Boolean algebras, we needed to assume certain local properties concerning centralizers of locally normal subgroups, the strongest of which was that $G$ should not have any nontrivial compact abelian locally normal subgroups. Fortunately, this last condition turns out to be true for a class of groups that includes every nondiscrete compactly generated topologically simple t.d.l.c. group.

For the sake of clarity, we shall first state and prove the absence of virtually abelian locally normal subgroups in groups belonging to the class $\mathscr{S}$. We then generalize this to the framework of Hecke pairs.

Theorem 5.3. Let $G \in \mathscr{S}$. Then $\mathrm{QZ}(G)=\{1\}$ and $G$ has no nontrivial virtually soluble locally normal subgroups. In particular $G$ is locally C-stable by Proposition 2.7. 
In particular, we recover [70, Theorem 2.2], which asserts that the compact open subgroups of $G$ cannot be soluble.

REMARK 5.4. Example 1.1 from the introduction (see also [70]) shows that there are nondiscrete topologically simple t.d.l.c. groups whose compact open subgroups are virtually abelian, so the condition of compact generation is essential in Theorem 5.3.

Proof of Theorem 5.3. That $\mathrm{QZ}(G)=\{1\}$ has already been proved in Proposition 4.4. We now suppose there is a nontrivial virtually soluble locally normal subgroup $K$ of $G$, in order to derive a contradiction. Then $K$ is nondiscrete, since $\mathrm{QZ}(G)=\{1\}$, so by intersecting $K$ with a sufficiently small compact open subgroup, we may assume that $K$ is soluble with compact closure. By passing to the last nontrivial term in the derived series, we may also assume that $K$ is abelian. Then $\bar{K}$ is also an abelian locally normal subgroup, so we may assume that $K$ is closed, hence compact. By Lemma 5.1, the group $G$ has a commensurated compact locally normal subgroup $L$ of the form $L=\prod_{i=1}^{n} L_{i}$, where the $L_{i}$ are locally normal, abelian (in fact they are each conjugate to an open subgroup of $K$ ), and normal in $L$. By Fitting's theorem, it follows that $L$ is nilpotent; in particular $\mathrm{Z}(L)>1$. On the other hand $L$ is an infinite, compact, commensurated, locally normal subgroup of $G$, and thus has a trivial quasicentralizer by Proposition 4.4. This is a contradiction.

We have now proved Theorem A; we can now also deduce Theorem $\mathrm{H}$ from Theorem 4.14, since the only missing ingredient was the fact that all groups in $\mathscr{S}$ are locally C-stable.

The conclusion of Theorem 5.3 may be extended to compactly generated topologically characteristically simple t.d.l.c. groups.

LEMMA 5.5 (See for instance [53, Lemma 8.2.3]). Let $G$ be a profinite group that is topologically characteristically simple. Then $G$ is a direct product of finite simple groups. In particular, $\mathrm{QZ}(G)$ is dense in $G$.

Proposition 5.6. Let $G$ be a compactly generated, topologically characteristically simple t.d.l.c. group. Then exactly one of the following holds:

(i) $G$ is finite.

(ii) $G$ is countably infinite and discrete.

(iii) $G$ is an infinite profinite group and $\mathrm{QZ}(G)$ is dense.

(iv) $G$ is locally $C$-stable and $\mathrm{QZ}(G)=\{1\}$. 
Proof. If $G$ is compact (which is to say that $G$ is profinite, since we have assumed $G$ is totally disconnected), it follows that $\mathrm{QZ}(G)$ is dense by Lemma 5.5. If $G$ is infinite and discrete, then it is finitely generated, hence countable. From now on we may assume $G$ is neither compact nor discrete.

By [18, Corollary D], there is a finite set of topologically simple closed normal subgroups $\left\{M_{1}, \ldots, M_{n}\right\}$, such that the product $\prod_{i=1}^{n} M_{i}$ is a dense subgroup of $G$, and $M_{i} \cap M_{j}=\{1\}$ for $i$ and $j$ distinct. Let $C_{i}=\mathrm{C}_{G}\left(M_{i}\right)$; note that $C_{i}$ contains $M_{j}$ for all $j \neq i$. We claim that $\mathrm{Z}(G)=\{1\}$ : otherwise, $G$ would be abelian and the $M_{i}$ would have to be cyclic of prime order, but then $G$ would itself be finite and hence discrete, a case we have already removed. Thus $M_{i} \cap C_{i}=\{1\}$ for $1 \leqslant i \leqslant n$. It follows that $G / C_{i}$ is topologically simple, since every nontrivial closed normal subgroup of $G / C_{i}$ has nontrivial intersection with $M_{i} C_{i} / C_{i}$ and is therefore dense in $G / C_{i}$. Thus $G / C_{i}$ is a locally C-stable t.d.l.c. group with trivial quasicentre by Theorem 5.3. Since the properties of being quasicentral and being abelian locally normal pass to quotients, it follows that $C_{i}$ contains $\mathrm{QZ}(G)$ and all abelian locally normal subgroups of $G$ for all $i$. Since $K=\bigcap_{i=1}^{n} C_{i}$ is a characteristic subgroup of $G$, it is trivial. We conclude that the quasicentre and all abelian locally normal subgroups of $G$ are trivial, hence $G$ is locally C-stable by Proposition 2.7.

In the case of an arbitrary compactly generated t.d.l.c. group $G$, as a result of Theorem 5.3, there is an interesting interaction between the [A]-regular radical of $G$, denoted by $\mathrm{R}_{[\mathrm{A}]}(G)$ (see [21, Theorem III]) and the quotients of $G$ occurring in Theorem 2.11 (which was borrowed from [18, Theorem A]).

COROLlARY 5.7. Let $G$ be a compactly generated t.d.l.c. group.

(i) Let $N$ be a cocompact normal subgroup of $G$ and $R$ be a closed normal subgroup of $N$ such that $N / R$ is nondiscrete and topologically simple. Then $N \cap \mathrm{R}_{[\mathrm{A}]}(G) \leqslant R$. Defining $N_{2}=\overline{N \mathrm{R}_{[\mathrm{A}]}(G)}$ and $R_{2}=\left\{g \in N_{2} \mid \forall h \in N\right.$ : $[g, h] \in R\}$, we have:

(a) $N_{2} / \mathrm{R}_{[\mathrm{A}]}(G)$ is a cocompact normal subgroup of $G / \mathrm{R}_{[\mathrm{A}]}(G)$;

(b) $R_{2}$ is a closed normal subgroup of $N_{2}$ which contains $\mathrm{R}_{[\mathrm{A}]}(G)$;

(c) $\mathrm{N}_{2} / R_{2}$ is nondiscrete, topologically simple and isomorphic to a quotient of $N_{2} / \mathrm{R}_{[\mathrm{A}]}(G)$; and

(d) the homomorphism $\phi: N / R \rightarrow N_{2} / R_{2}$ given by $\phi(g R)=g R_{2}$ is injective and has dense image.

All nondiscrete simple quotients of cocompact normal subgroups of $G$ are thus accounted for by the quotient $G / \mathrm{R}_{[\mathrm{A}]}(G)$. 
(ii) Suppose $G$ is noncompact and $[\mathrm{A}]$-regular (that is $G=\mathrm{R}_{[\mathrm{A}]}(G)$ ). Then $G$ has an infinite discrete quotient.

Proof. (i) Since $N$ is cocompact in $G$, it is compactly generated, and hence so is the topologically simple quotient $N / R$. From Theorem 5.3, we deduce that $\mathrm{R}_{[\mathrm{A}]}(N / R)=\{1\}$, so $\mathrm{R}_{[\mathrm{A}]}(N) \leqslant R$ by [21, Theorem 6.11]. By [21, Proposition 6.15], we have $\mathrm{R}_{[\mathrm{A}]}(G) \cap N \leqslant \mathrm{R}_{[\mathrm{A}]}(N)$, and hence $N \cap \mathrm{R}_{[\mathrm{A}]}(G) \leqslant R$. Let $N_{2}=\overline{N \mathrm{R}_{[\mathrm{A}]}(G)}$ and $R_{2}=\left\{g \in N_{2} \mid \forall h \in N:[g, h] \in R\right\}$. Clearly $N_{2}$ is normal in $G$. Moreover $N_{2}$ is cocompact in $G$, since it contains $N$, and so $N_{2} / \mathrm{R}_{[\mathrm{A}]}(G)$ is a cocompact normal subgroup of $G / \mathrm{R}_{[\mathrm{A}]}(G)$. Note that $R_{2}$ is a closed subgroup of $N_{2}$, since it is the set of all $g \in N_{2}$ such that $g R \in \mathrm{C}_{G / R}(N / R)$. We have $\left[N, \mathrm{R}_{[\mathrm{A}]}(G)\right] \leqslant N \cap \mathrm{R}_{[\mathrm{A}]}(G) \leqslant R$, and so $\mathrm{R}_{[\mathrm{A}]}(G) \leqslant R_{2}$. Since $R$ is normal in $N$, we see that $R_{2}$ is normalized by $N$ and thus $R_{2}$ is normal in $N_{2}$, so $\phi$ is a well-defined homomorphism; the fact that $\mathrm{R}_{[\mathrm{A}]}(G) \leqslant R_{2}$ ensures that $\phi$ has dense image and that $N_{2} / R_{2}$ is isomorphic to a quotient of $N_{2} / \mathrm{R}_{[\mathrm{A}]}(G)$. Since the centre of $N / R$ is trivial, we see that $N \cap R_{2}=R$, and hence that the homomorphism $\phi$ is injective. Since $\phi$ is continuous and injective and $N / R$ is nondiscrete, it follows that $N_{2} / R_{2}$ is not discrete.

It remains to show that $N_{2} / R_{2}$ is topologically simple. Let $K / R_{2}$ be a proper closed normal subgroup of $N_{2} / R_{2}$. Then $K$ does not contain $N$, and it follows that $\phi^{-1}\left(K / R_{2}\right)$ is a proper closed normal subgroup of $N / R$ and, by topological simplicity of $N / R$, must be equal to $\{1\}$. Thus $K / R_{2}$ and $N R_{2} / R_{2}$ are normal subgroups of $N_{2} / R_{2}$ with trivial intersection. We conclude that $K / R_{2}$ centralizes $N R_{2} / R_{2}$, that is, $[g, h] \in R_{2}$ for all $g \in K$ and $h \in N$. Since $[g, h] \in N$ as well in this case, in fact $[g, h] \in N \cap R_{2}=R$ and so $K \leqslant R_{2}$. Thus $K / R_{2}=\{1\}$, as required.

(ii) Follows from (i) and Theorem 2.11.

We now proceed to generalize Theorem 5.3 to the abstract framework of Hecke pairs.

THEOREM 5.8. Let $(G, U)$ be a Hecke pair such that $U<G$ and such that $U$ is infinite. Suppose that $G$ is generated by finitely many cosets of $U$, and also that $G=\langle U, N\rangle$ for any nontrivial normal subgroup $N$ of $G$.

Then the following assertions hold for any nontrivial subgroup $K$ of $U$ such that $\mathrm{N}_{U}(K)$ has finite index in $U$ :

(i) $K$ is not virtually soluble.

(ii) If $K$ is commensurated by $G$, then $\mathrm{C}_{G}(K)=\{1\}$. 
Proof. Let $K$ be a nontrivial subgroup of $U$ such that $\left|U: \mathrm{N}_{U}(K)\right|$ is finite.

Let us first prove (ii) in case $K$ is infinite. Suppose for a contradiction that $C=\mathrm{C}_{G}(K)>\{1\}$. Then $G$ is generated by $U$ together with a finite set $\left\{g_{1} C g_{1}^{-1}\right.$, $\left.\ldots, g_{n} C g_{n}^{-1}\right\}$ of conjugates of $C$, and without loss of generality we may assume that $g_{1}=1$. Moreover $U$ has finite orbits on the set of conjugates of $K$, since $\mathrm{N}_{U}(K)$ is a commensurated subgroup of $G$ that has finite index in $U$. Hence we may take $\left\{g_{1} K g_{1}^{-1}, \ldots, g_{n} K g_{n}^{-1}\right\}$ to be a $U$-invariant set. Consequently the group $L=\bigcap_{i=1}^{n} g_{i} K g_{i}^{-1}$ is normal in $G$, since it is normalized by $U$ and centralized by $\left\langle g_{1} C g_{1}^{-1}, \ldots, g_{n} C g_{n}^{-1}\right\rangle$. Since $L \leqslant U$ (because $L \leqslant g_{1} K g_{1}^{-1}=K \leqslant U$ ), we conclude that $L=\{1\}$, so $K$ cannot have been an infinite commensurated subgroup of $G$. This proves (ii) in the case where $K$ is infinite.

If $K$ is finite, we see that $L=\mathrm{C}_{U}(K)$ has finite index in $U$ and is thus an infinite commensurated subgroup of $G$. What we have just proved ensures that $\mathrm{C}_{G}(L)=\{1\}$, so that $K=\{1\}$, a contradiction. This completes the proof of (ii).

This implies that $\mathrm{C}_{G}(V)=\{1\}$ for every subgroup $V$ of $G$ that is commensurate with $U$.

We now prove (i), and assume thus that $K$ is virtually soluble. The fact that $\mathrm{C}_{G}(V)=\{1\}$ for every finite index subgroup $V$ of $U$ ensures that $\mathrm{C}_{U}(K)$ has infinite index in $\mathrm{N}_{U}(K)$ and hence that $K$ must be infinite. Moreover, the hypotheses imply that $U$ has trivial core in $G$. In particular $U$ is residually finite, so there is an injective map $\pi: U \rightarrow \hat{U}$ from $U$ to its profinite completion. We see that $\overline{\pi(K)}$ has a soluble closed subgroup of finite index; by a compactness argument, there exists an open normal subgroup $W$ of $\hat{U}$ such that $\overline{\pi(K)} \cap W$ is soluble. The group $K^{\prime}=\pi^{-1}(W) \cap K$ is then a soluble subgroup of finite index in $K$, such that $K^{\prime}$ is normalized by $\mathrm{N}_{U}(K)$. Therefore, in order to prove (i), we may assume henceforth that $K$ is soluble and derive a contradiction. By replacing $K$ with the last nontrivial term of its derived series, we may indeed assume that $K$ is abelian. We have $G=\langle U, \kappa\rangle$ where $\kappa$ is the set of $G$-conjugates of $K$. By Lemma 5.1, there is a set $\left\{L_{1}, \ldots, L_{n}\right\}$ of subgroups of $G$, each a finite index subgroup of a conjugate of $K$, such that $L_{i}$ and $L_{j}$ normalize each other for all pairs $(i, j)$ and $L=\prod_{i=1}^{n} L_{i}$ is an infinite normal subgroup of $U$ that is commensurated by $G$. Now each $L_{i}$ is abelian, so $L$ is nilpotent by Fitting's theorem, and hence $\mathrm{C}_{G}(L) \geqslant \mathrm{Z}(L)>1$. This is impossible, as we have already shown that any infinite normal subgroup of $U$ that is commensurated by $G$ must have a trivial centralizer.

It is straightforward to deduce Theorem 5.3 above from Theorem 5.8, thereby providing an alternative proof of the former. The following particularization of Theorem 5.8, whose proof is straightforward, is another special case of independent interest: 
COROLLARY 5.9. Let $G$ be a finitely generated simple group with an infinite commensurated subgroup $U$. Then $\mathrm{C}_{G}(U)=\{1\}$, and $U$ does not have any virtually soluble normal subgroups except for the trivial group.

5.3. Five possible types of structure lattice. Our aim in this subsection is to prove Theorem F. We first make an easy observation about the structure of $\mathcal{L} \mathcal{N}(G) \backslash\{0\}$.

An upper subset of a poset $X$ is a subset $Y$ such that for all $y \in Y$ and $x \in X$, if $y \leqslant x$ then $x \in Y$. A filter of a lattice is an upper subset that is also closed under meets.

LEMMA 5.10. Let $G$ be a nondiscrete locally $C$-stable t.d.l.c. group and let $\mathcal{F}:=$ $\mathcal{L N}(G) \backslash\{0\}$. Then $\mathcal{F}$ is closed under meets if and only if $\mathcal{L C}(G)=\{0, \infty\}$.

Proof. $\mathcal{F}$ is clearly an upper subset of $\mathcal{L} \mathcal{N}(G)$, so it is a filter if and only if it is closed under meets.

If $\mathcal{L C}(G)$ is nontrivial, then there exists an infinite compact locally normal subgroup $K$ of $G$ such that $\mathrm{C}_{G}(K)$ is infinite. Then $\alpha=[K]$ and $\beta=\left[\mathrm{C}_{G}(K)\right]$ are elements of $\mathcal{F}$ such that $[K] \wedge\left[\mathrm{C}_{G}(K)\right]=[\mathrm{Z}(K)]=0$, recalling that $G$ has no nondiscrete abelian locally normal subgroups by Proposition 2.7. Thus $\mathcal{F}$ is not closed under meets. Conversely, if there exist $\alpha, \beta \in \mathcal{F}$ such that $\alpha \wedge \beta=0$, then we have $0<\beta \leqslant \alpha^{\perp}<\infty$, so $\alpha^{\perp}$ is an element of $\mathcal{L C}(G)$ other than 0 and $\infty$.

We now show that for $G \in \mathscr{S}$, the set of nonzero fixed points of $G$ acting on $\mathcal{L N}(G)$ forms a filter, even if $\mathcal{L C}(G)$ is nontrivial.

LEMMA 5.11. Let $G$ be a nondiscrete t.d.l.c.s.c. group.

(i) If $G$ is topologically simple, then $\mathcal{L N}(G)^{G} \backslash\{0\}$ is an upper subset of $\mathcal{L N}(G)$.

(ii) If $G$ is monolithic, locally $C$-stable and has trivial quasicentre, then $\mathcal{L} \mathcal{N}(G)^{G} \backslash\{0\}$ is closed under meets.

Hence if $G$ is topologically simple and locally $C$-stable, then $\mathcal{L N}(G)^{G} \backslash\{0\}$ is a filter on $\mathcal{L N}(G)$.

Proof. Let $\mathcal{F}=\mathcal{L} \mathcal{N}(G)^{G} \backslash\{0\}$.

(i) Let $K$ be a compact locally normal subgroup of $G$ such that $[K] \in \mathcal{F}$. Then $G$ acts on the set $\mathcal{R}$ of elements of $\mathcal{L N}(G)$ above [K]; the kernel $R$ of this action is closed and normal in $G$. Moreover $R$ contains $K$, since for every $\beta>[K]$, there 
is a representative $L$ of $\beta$ containing $K$. Hence $R \geqslant \overline{\langle\langle K\rangle}$. Since $G$ is topologically simple, we conclude that $R=G$, so $G$ acts trivially on $\mathcal{R}$. Thus $\beta>[K]$ implies $\beta \in \mathcal{F}$.

(ii) Note that the hypotheses ensure $\mathrm{QZ}(H)=\{1\}$ for every nontrivial closed locally normal subgroup $H$ of $G$ by Proposition 2.7. We suppose that $\alpha, \beta \in \mathcal{F}$ are such that $\alpha \wedge \beta=0$; it suffices to derive a contradiction.

Let $M$ be the monolith of $G$ and let $\mu=[M] \in \mathcal{L N}(G)$. Then $\mu \in \mathcal{L N}(G)^{G}$, so $\gamma \wedge \mu \in \mathcal{L N}(G)^{G}$ for all $\gamma \in \mathcal{F}$. Moreover we have $\gamma \wedge \mu>0$ for all $\gamma \in \mathcal{F}$, since otherwise $M$ would have a nontrivial quasicentralizer in $G$, contradicting Proposition 4.3. In particular, we infer that $\alpha \wedge \mu$ and $\beta \wedge \mu$ both belong to $\mathcal{F}$. Since $\alpha \wedge \beta=0$, there are infinite compact subgroups $K$ and $L$ of $M$ such that $K \in \alpha \wedge \mu, L \in \beta \wedge \mu, K$ and $L$ normalize each other and $K \cap L=\{1\}$, so $L \leqslant \mathrm{C}_{M}(K)$. The desired contradiction follows by Proposition 4.3. In particular $\alpha \wedge \beta>0$, so $\alpha \wedge \beta \in \mathcal{F}$.

We immediately derive an important property of the action of $G$ on $\mathcal{L C}(G)$.

COROLlary 5.12. Let $G$ be a topologically simple, locally $C$-stable t.d.l.c.s.c. group. Then $\mathcal{L C}(G)^{G}=\{0, \infty\}$.

Proof. Let $\alpha \in \mathcal{L C}(G)^{G}$ and suppose that $\alpha \notin\{0, \infty\}$. Since the map $\perp: \mathcal{L C}(G) \rightarrow \mathcal{L C}(G)$ is invariant under the $G$-action, it follows that $\alpha^{\perp}$ is also fixed by $G$; at the same time, $\alpha^{\perp} \notin\{0, \infty\}$. Thus $\alpha$ and $\alpha^{\perp}$ are nonzero elements of $\mathcal{L N}(G)^{G}$. It follows from Lemma 5.11(ii) that $\alpha \wedge \alpha^{\perp}>0$; however, $\alpha \wedge \alpha^{\perp}=0$ by Theorem 2.10.

Theorem $\mathrm{F}$ is now straightforward to prove.

Proof of Theorem F. It is clear that the five types are mutually exclusive.

Let $G \in \mathscr{S}$. Since $G$ is nondiscrete, we have $|\mathcal{L N}(G)| \geqslant 2$. By Theorem 5.3, we have $\mathrm{QZ}(G)=\{1\}$ and $G$ has no nontrivial abelian locally normal subgroup, so $\mathcal{L C}(G)$ and $\mathcal{L D}(G)$ are Boolean algebras by Theorem 2.10.

Suppose $|\mathcal{L N}(G)|=2$. Then $\mathcal{L} \mathcal{N}(G)=\{0, \infty\}$, so every compact locally normal subgroup of $G$ is finite or open. Moreover, $G$ has no nontrivial finite locally normal subgroups since $\mathrm{QZ}(G)=\{1\}$. In particular, given any compact open subgroup $U$ of $G$, then every nontrivial closed locally normal subgroup of $U$ is open, in other words $U$ is h.j.i. Thus $G$ is locally h.j.i. From now on we assume $|\mathcal{L N}(G)|>2$.

Suppose that $\mathcal{L C}(G)=\{0, \infty\}$. Then $\mathcal{F}$ is a filter by Lemma 5.10. If $\mathcal{F}$ is a principal filter then the least element of $\mathcal{F}$ must be fixed by $G$, by uniqueness; consequently by Lemma 5.11, $G$ acts trivially on $\mathcal{F}$ and hence on $\mathcal{L N}(G)$. 
The fact that $G$ has nontrivial fixed points on $\mathcal{L N}(G)$ ensures that $G$ does not satisfy (S2), so by Theorem 3.9, $G$ is not abstractly simple. Thus $G$ is of atomic type in this case. If instead $\mathcal{F}$ is a nonprincipal filter, then $G$ is of NPF type.

Suppose that $\mathcal{L C}(G)$ is nontrivial. If $\mathcal{L D}(G)=\{0, \infty\}$ then $G$ is weakly decomposable, otherwise $G$ is locally decomposable.

Given the list of types, it is clear that we can recover the type of $G$ from the isomorphism type of the poset $\mathcal{L N}(G)$.

\subsection{Dense normal subgroups of topologically simple groups. In this} subsection, $G$ is a nondiscrete t.d.l.c.s.c. group. Recall that $\mathcal{L N}(G)^{G}$ is the set of fixed points of the action of $G$ on $\mathcal{L N}(G)$, in other words the set of local equivalence classes of commensurated compact locally normal subgroups of $G$. Evidently $\mathcal{L} \mathcal{N}(G)^{G}$ is a sublattice of $\mathcal{L N}(G)$ and contains both 0 and $\infty$. By Theorem 3.9, if $G$ is abstractly simple then $\mathcal{L N}(G)^{G}=\{0, \infty\}$. Thus if $G$ is topologically simple, the existence of a nontrivial element of $\mathcal{L N}(G)^{G}$ implies the existence of a proper dense normal subgroup of $G$. However, we do not know if such a nontrivial fixed point can exist. Our goal in this subsection and the next is to obtain restrictions on this situation by describing the structure of the dense normal subgroups that could arise, leading in particular to Theorem C.

Before stating our results, we recall some terminology and results that will be used in this subsection and the next.

LEMMA 5.13. Let $G$ be a t.d.l.c. group and let $\mu$ be a commensurability class (or subset of a commensurability class) of compact subgroups of $G$. Let $K \in$ $\mu$ and consider $\operatorname{Comm}_{G}(K)$. Then there is a unique group topology $\mathcal{T}_{(\mu)}$ on $\operatorname{Comm}_{G}(K)$ such that the inclusion $L \rightarrow \operatorname{Comm}_{G}(K)$ is continuous and open, for every compact subgroup $L$ of $G$ commensurate with $K$. Moreover, the group $\operatorname{Comm}_{G}(K)$ and the topology are uniquely determined by the pair $(G, \mu)$.

Proof. Without loss of generality, we assume that $\mu$ consists of all compact subgroups of $G$ commensurate with $K$. Note that given $L \in \mu$, then $\operatorname{Comm}_{G}(K)=\operatorname{Comm}_{G}(L)$, so in particular $L \leqslant \operatorname{Comm}_{G}(K)$, and the subgroup $\operatorname{Comm}_{G}(K)$ of $G$ is uniquely determined by $(G, \mu)$. We define $\mathcal{T}_{(\mu)}$ to be the topology generated by the left cosets of elements of $\mu$; observe that the inclusion $L \rightarrow \operatorname{Comm}_{G}(K)$ is then continuous and open, for every $L \in \mu$. Given Lemma 3.13, it is clear that $\operatorname{Comm}_{G}(K)$ acts by homeomorphisms on itself by left and right translation. Given $g \in \operatorname{Comm}_{G}(K)$ and $L \in \mu$, the preimage of $g L$ under the map $(x, y) \mapsto x y^{-1}$ is a union of sets of the form $(g L h, L h)$ for $h \in \operatorname{Comm}_{G}(K)$; such sets are open by the fact that left and right translation are homeomorphisms. Thus $\mathcal{T}_{(\mu)}$ is a group topology. 
The uniqueness of the topology follows from the general observation that a group topology is uniquely determined by its restriction to a neighbourhood of the identity, which in this case is determined by the topology of $K$.

DEFINITION 5.14. Let $G$ be a t.d.l.c. group and let $\mu$ be a commensurability class (or subset of a commensurability class) of compact subgroups of $G$. The localization $G_{(\mu)}$ of $G$ at $\mu$ is the topological group $\left(\operatorname{Comm}_{G}(K), \mathcal{T}_{(\mu)}\right)$, where $K$ is any element of $\mu$.

Starting with a first-countable t.d.l.c. group $G$, one sees that the t.d.l.c. groups with the same group structure as $G$ but a finer topology are precisely the groups $G_{(\mu)}$, where $\mu$ is a $G$-invariant commensurability class of compact subgroups of $G$. Indeed, we recall by Lemma 2.4 that it suffices to consider $G$-invariant commensurability classes of compact locally normal subgroups of $G$.

Definition 5.15. Following Abels [1], we say that a locally compact group $G$ is compactly presented if there is a surjective homomorphism $\theta: F_{X} \rightarrow G$, where $F_{X}$ is the abstract free group on the set $X$, so that $\theta(X)$ is compact in $G$ and the kernel of $\theta$ is generated by words in the alphabet $X \cup X^{-1}$ of bounded length.

Many well-known properties of finitely presented discrete groups carry over to compactly presented locally compact groups (see [24, Ch. 8] for a detailed account). A sufficient condition for $G$ to be compactly presented is that $G$ admits some continuous, proper cocompact action on a simply connected proper geodesic metric space (see [24, Corollary 8.A.9]). In particular any centreless simple Lie group $G$ is compactly presented, since the coset space $G / K$ modulo a maximal compact subgroup $K$ is contractible.

LEMMA 5.16 [24, Proposition 8.A.10]. Let $\varphi: \tilde{G} \rightarrow G$ be a continuous surjective homomorphism of locally compact groups, whose kernel $\operatorname{Ker}(\varphi)$ is discrete. If $G$ is compactly presented and $\tilde{G}$ is compactly generated, then $\operatorname{Ker}(\varphi)$ is finitely generated as a normal subgroup of $\tilde{G}$.

Let $\alpha \in \mathcal{L} \mathcal{N}(G)^{G} \backslash\{0\}$, let $L$ be a compact locally normal representative of $\alpha$ and let $D=\langle\langle L\rangle\rangle$, equipped with the $\mathcal{T}_{(\alpha)}$-subspace topology. Then $D$ is a $\mathcal{T}_{(\alpha)}{ }^{-}$ open subgroup of $G$. Moreover, equipping the direct product $G \times D$ with the product topology, then by [21, Proposition 7.9], the map

$$
G \times D \rightarrow D ; \quad(g, d) \mapsto g d g^{-1}
$$

is continuous. This ensures that the natural semidirect product $D \rtimes G$ is a topological group when equipped with the product topology, which allows us to 
analyse the structure of the embedding $D \hookrightarrow G$, especially when $D$ is dense in $G$ (as is certainly the case if $G$ is topologically simple).

Proposition 5.17. Let $G$ be a nondiscrete t.d.l.c.s.c. group and $L$ be an infinite commensurated compact locally normal subgroup of $G$. Let $\alpha=[L]$ and $D=$ $\left\langle\langle L\rangle\right.$ be the abstract normal closure of $L$ in $G$, equipped with the $\mathcal{T}_{(\alpha)}$-subspace topology. Let $\psi: D \rightarrow G$ be the natural inclusion map and let $U$ be a compact open subgroup of $\mathrm{N}_{G}(L)$ containing $L$. Suppose that $D$ is dense in $G$.

Then there is a t.d.l.c. group $\tilde{G}$, a closed continuous injective homomorphism $\iota: D \rightarrow \tilde{G}$, and a quotient homomorphism $\pi: \tilde{G} \rightarrow G$ such that

(1) $\psi=\pi \circ \iota$;

(2) $\iota(D)$ is a closed cocompact normal subgroup of $\tilde{G}$, with $\tilde{G} / \iota(D) \cong U / L$;

(3) $\operatorname{Ker}(\pi)$ is discrete and centralizes $\iota(D)$;

(4) Every element of $\operatorname{Ker}(\pi)$ lies in a finite conjugacy class of $\tilde{G}$;

(5) $\tilde{G}=\overline{\iota(D) \operatorname{Ker}(\pi)}$;

(6) If $\operatorname{Ker}(\pi)$ or $U / L$ is virtually abelian, then $D \geqslant[G, G]$.

Proof. Form $G^{\rtimes}=D \rtimes U$ where $U$ acts on $D$ by conjugation, and equip $G^{\rtimes}$ with the product topology. By [21, Proposition 7.9], the action of $U$ on $D$ is continuous, so that $G^{\rtimes}$ is a topological group. There is also a natural inclusion $\iota_{1}: D \rightarrow G^{\rtimes}$, which is a closed embedding with cocompact image.

Given a subgroup $K$ of $U$, let

$$
\Delta_{K}:=\left\{\left(k^{-1}, k\right) \in G^{\rtimes} \mid k \in K \cap D\right\} .
$$

The set $\Delta_{K}$ is a subgroup of $G^{\rtimes}$ that centralizes $\iota_{1}(D)$, and if $\psi^{-1}(K)$ is compact, then $\Delta_{K}$ is compact. If $K$ is normal in $U$, it follows further that $\Delta_{K}$ is normal in $G^{\rtimes}$. In particular, the set $\Delta_{L}$ is a compact normal subgroup of $G^{\rtimes}$.

We now set

$$
\tilde{G}:=G^{\rtimes} / \Delta_{L}
$$

and let $\rho: G^{\rtimes} \rightarrow \tilde{G}$ be the usual projection. Note that $\rho$ is a quotient map with compact kernel, so it is a closed map. Set $\iota:=\rho \circ \iota_{1}$; it is immediately clear that $\iota$ is continuous and injective. Moreover, $\iota$ is a closed map, since $\iota_{1}$ and $\rho$ are both closed. Thus $\iota$ is a closed embedding.

The homomorphism $\pi: \tilde{G} \rightarrow G$ is defined by

$$
\pi\left((d, u) \Delta_{L}\right)=\psi(d) u .
$$


The image of $\pi$ is dense and contains the open subgroup $U$ of $G$, hence the map is surjective. It is easy to check this map is also continuous. Since $D$ and $G$ are second-countable, so are $G^{\rtimes}$ and $\tilde{G}$. By [3, Corollary to Theorem 8], a continuous surjective homomorphism between second-countable locally compact groups is open. Therefore, the homomorphism $\pi$ is open, hence a quotient map, and $G$ isomorphic to the quotient $\tilde{G} / \operatorname{Ker}(\pi)$. Setting $\tilde{U}:=\rho(U)$, the group $\tilde{U}$ is a compact open subgroup of $\tilde{G}$ since $\rho(U)=\rho\left(U \Delta_{L}\right)$ and since the group $U \Delta_{L}=L \rtimes U$ is open in $G^{\rtimes}$. Furthermore, $\tilde{U} \cap \operatorname{Ker}(\pi)=\{1\}$, hence $\operatorname{Ker}(\pi)$ is discrete.

We now check the desired properties hold of $\tilde{G}, \iota$ and $\pi$. Part (1) is immediate. That $\iota(D)$ is cocompact follows since $\rho$ induces a continuous surjective map $G^{\rtimes} / \iota_{1}(D) \rightarrow \tilde{G} / \iota(D)$. We thus have verified (2).

The kernel of $\pi$ is exactly $K:=\Delta_{U} / \Delta_{L}$. In particular, we see that $K$ is discrete. The group $\Delta_{U}$ is centralized by $\iota_{1}(D)$, hence $K$ is centralized by $\iota(D)$, verifying (3). We conclude that $\mathrm{C}_{\tilde{G}}(K)$ is cocompact in $\tilde{G}$. On the other hand, $K$ is a discrete normal subgroup of $\tilde{G}$, so $\mathrm{C}_{\tilde{G}}(x)$ is open in $\tilde{G}$ for every $x \in K$. Consequently, for every $x \in K$, then $\mathrm{C}_{\tilde{G}}(x)$ is both cocompact and open, so it has finite index in $\tilde{G}$, verifying (4).

Since $\pi$ is a quotient map and $\psi(D)$ is dense in $G$, we see that $\pi^{-1}(\psi(D))$ is dense in $\tilde{G}$. In other words, $\iota(D) \operatorname{Ker}(\pi)$ is dense in $\tilde{G}$, verifying (5).

We see from properties (2) and (5) that $\operatorname{Ker}(\pi)$ is isomorphic to a dense subgroup of $U / L$, so $\operatorname{Ker}(\pi)$ is virtually abelian if and only if $U / L$ is virtually abelian. Suppose that $U / L$ is virtually abelian, in other words, there exists a compact open subgroup $V$ of $U$ such that $L \leqslant V$ and $V / L$ is abelian. We now perform the same construction of $\tilde{G}$ as before, with $V$ in place of $U$. Now since $V / L$ is abelian, property (2) ensures that $\tilde{G} / \iota(D)$ is abelian, so $\iota(D)$ contains the derived group of $G$; since $G=\pi(\tilde{G})$, it follows that $D=\pi(\iota(D)) \geqslant[G, G]$, proving (6).

If $G$ is compactly presented, we obtain a stronger form of Proposition 5.17.

Proposition 5.18. Retain the hypotheses of Proposition 5.17, and assume in addition that $G$ is compactly presented. Then $D \geqslant[G, G]$. Moreover, there is a t.d.l.c. group $\tilde{G}$, a closed continuous injective homomorphism $\iota: D \rightarrow \tilde{G}$, and a quotient homomorphism $\pi: \tilde{G} \rightarrow G$ such that the conditions in Proposition 5.17 hold, and in addition:

(1) $\operatorname{Ker}(\pi)$ commutes with a finite index subgroup of $\tilde{G}$ that contains $\iota(D)$;

(2) Both $\operatorname{Ker}(\pi)$ and $U / L$ are centre-by-finite, finite-by-abelian and topologically finitely generated. 
Proof. We retain the construction and notation of Proposition 5.17.

We have assumed that $G$ is compactly presented. In particular, $G$ is compactly generated, so $D$ is compactly generated by Lemma 4.2. Since $\iota_{1}(D)$ is cocompact in $\tilde{G}$, it follows that $\tilde{G}$ is compactly generated. We can now apply Lemma 5.16 to conclude that $\operatorname{Ker}(\pi)=\langle\langle X\rangle$ for a finite subset $X$ of $G$. By Proposition 5.17(4), each element of $X$ only has finitely many $\tilde{G}$-conjugates, so $\operatorname{Ker}(\pi)=\langle Y\rangle$ for a finite set $Y$. Let $C=\bigcap_{y \in Y} \mathrm{C}_{\tilde{G}}(y)$, so that $C$ centralizes $\operatorname{Ker}(\pi)$. Then $C$ is closed in $\tilde{G}$ (since every centralizer is closed) and of finite index (since each element of $Y$ has a finite conjugacy class); also $C$ contains $\iota(D)$ by Proposition 5.17(3). Thus (1) holds.

It follows that $C$ is open in $\tilde{G}$ and $C \iota(D) / \iota(D)$ is an open subgroup of $\tilde{G} / \iota(D)$ of finite index. By property (5), the image of $\operatorname{Ker}(\pi)$ in $\tilde{G} / \iota(D)$ is dense, so $C \iota(D) / \iota(D)$ has a dense centralizer in $\tilde{G} / \iota(D)$; consequently, it is central in $\tilde{G} / \iota(D)$. Thus $\tilde{G} / \iota(D)$ is centre-by-finite; since $\tilde{G} / \iota(D) \cong U / L$, it follows that $U / L$ is centre-by-finite. Since $\operatorname{Ker}(\pi)$ has dense image in $\tilde{G} / \iota(D)$, it follows that $\tilde{G} / \iota(D)$ is topologically finitely generated, so $U / L$ is topologically finitely generated.

By a classical result of Schur [57] (see also [55] for a short proof of a stronger result), every centre-by-finite group is finite-by-abelian. Thus $\operatorname{Ker}(\pi)$ and $U / L$ are also finite-by-abelian. Thus (2) holds.

Since $\operatorname{Ker}(\pi)$ is virtually abelian, it follows from Proposition 5.17(6) that $D \geqslant$ $[G, G]$.

We can now establish a particular circumstance in which there are no nontrivial fixed points in the structure lattice of $G$.

Corollary 5.19. Let $G \in \mathscr{S}$. Assume that $G$ is compactly presented. If $G$ is abstractly perfect, or if some compact open subgroup $U$ of $G$ is such that $U / \overline{[U, U]}$ is finite, then $\mathcal{L} \mathcal{N}(G)^{G}=\{0, \infty\}$.

Proof. Let $\alpha \in \mathcal{L} \mathcal{N}(G)^{G} \backslash\{0\}$, let $L$ be a compact locally normal representative of $\alpha$ and let $D=\langle\langle L\rangle$. Then by Proposition 5.18, we have $D \geqslant[G, G]$ and $U / L$ is finite-by-abelian. If $G=[G, G]$, it follows that $D=G$. Hence $G$ is $L$-meagre by Lemma 3.14; in other words, the index of $L$ in $G$ is countable. By the Baire Category Theorem, it follows that $L$ is open in $G$. If $U / \overline{[U, U]}$ is finite, then $U / L$ must be finite, so $L$ is open in $U$ and hence in $G$. In either case, $L$ is open in $G$, so $\alpha=\infty$. This proves that $\mathcal{L} \mathcal{N}(G)^{G}=\{0, \infty\}$ under the given hypotheses.

Given $G \in \mathscr{S}$ and $D$ as in Proposition 5.17, it is not known if $D$ can be a proper subgroup of $G$; assuming $D$ is a proper subgroup, it is not even clear whether or 
not $D \in \mathscr{S}$. However, we can show that the closure in the $\mathcal{T}_{(\alpha)}$-topology of the derived group of $D$ belongs to the class $\mathscr{S}$.

Proposition 5.20. Let $G \in \mathscr{S}$, let $\alpha \in \mathcal{L} \mathcal{N}(G)^{G} \backslash\{0\}$, let $L$ be a compact locally normal representative of $\alpha$, and let $D=\langle\langle L\rangle\rangle$ be the abstract normal closure of $L$ in $G$, equipped with the $\mathcal{T}_{(\alpha)}$-subspace topology. Let $S=\overline{[D, D]}$, where the closure is taken with respect to the topology of $D$. Then $S \in \mathscr{S}$. Moreover, $S=\langle\langle M\rangle\rangle$ for some infinite commensurated compact locally normal subgroup $M$ of $G$ contained in $L$, and the topologies $\mathcal{T}_{(\alpha)}$ and $\mathcal{T}_{(\beta)}$ coincide on $S$, where $\beta=[M]$.

Proof. Form the semidirect product $G^{\rtimes}=D \rtimes G$, equipped with the product topology. As observed before Proposition 5.17, $G^{\rtimes}$ is a topological group. In particular, given any $\mathcal{T}_{(\alpha)}$-closed subgroup $K$ of $D$ such that $\mathrm{N}_{G}(K)$ is dense in $G$, then $K \rtimes\{1\}$ is a closed subgroup of $G^{\rtimes}$ that is normalized by a dense subgroup of $\{1\} \rtimes G$, hence $K \rtimes\{1\}$ is normalized by all of $\{1\} \rtimes G$ (since the normalizer of any closed subgroup is closed), and so $K$ is normal in $G$.

By Theorem 5.3, the group $G$ has no nontrivial virtually abelian locally normal subgroups. Consequently, both $L$ and $\overline{[L, L]}$ are nontrivial, hence non-(virtually abelian). This ensures that $S \geqslant \overline{[L, L]}$ is nondiscrete. We now claim that any closed subgroup $K$ of $D$ that is normalized by $S=\overline{[D, D]}$ must satisfy $K \geqslant S$. This will ensure in particular that $S$ is topologically simple.

Let $K$ be a closed subgroup of $D$ such that $S \leqslant \mathrm{~N}_{D}(K)$. Since $S$ is a closed normal subgroup of $D$, we deduce from the first paragraph of the proof above that $S$ is normal in $G$. Since $S$ is nontrivial and $G$ is topologically simple, it follows that $S$ itself is dense in $G$. In turn, this means that $K$ is normalized by a dense subgroup of $G$, so using again the first paragraph above, we infer that $K$ is normal in $G$, and hence $K$ is dense in $G$.

In $G^{\rtimes}$, both $K^{*}:=K \rtimes\{1\}$ and $\Delta_{K}:=\left\{\left(k^{-1}, k\right) \in G^{\rtimes} \mid k \in K\right\}$ are closed normal subgroups. Since $K$ is dense in $G$, we observe that $K^{*} \Delta_{K}$ is a dense normal subgroup of $G^{\rtimes}$. Moreover, $\Delta_{K}$ centralizes $D^{*}:=D \rtimes\{1\}$. Thus in the quotient $G^{\rtimes} / K^{*}$, we see that $D^{*} / K^{*}$ is a subgroup with dense centralizer, so it is central in $G^{\rtimes} / K^{*}$. In particular, $D^{*} / K^{*}$ is abelian. Since $D^{*} / K^{*} \cong D / K$, it follows that $D / K$ is abelian, that is, $[D, D] \leqslant K$. Since $K$ is closed by assumption, in fact $S \leqslant K$, and the claim is proven.

Let $M$ be a compact subgroup of $S$ relatively open for the $\mathcal{T}_{(\alpha)}$-topology, and such that $M \leqslant L$. Then $S=\langle\langle M\rangle\rangle$, since $S$ is topologically simple. Moreover, since $M$ is relatively $\mathcal{T}_{(\alpha)}$-open, it follows that the topologies $\mathcal{T}_{(\alpha)}$ and $\mathcal{T}_{(\beta)}$ coincide on $S$, where $\beta=[M]$. Since the conjugation action of $G$ respects the topology of $D$, and hence also the topology of its closed subgroup $S$, we see that $M$ is 
commensurated in $G$. By Lemma 2.4, by replacing $M$ with a finite index open subgroup of $M$, we may ensure that $M$ is locally normal in $G$. Finally, we conclude from Lemma 4.2 that $S$ is compactly generated. Thus $S$ is in $\mathscr{S}$.

Suppose that there is a compact open subgroup $U$ of $G$ that is topologically finitely generated. In this case, we can use a special case of far-reaching results due to Nikolov and Segal [48] (the proof of which relies on the classification of the finite simple groups) to restrict the structure of dense normal subgroups of $G$, and hence that of commensurated compact locally normal subgroups. (We attribute the next result to Nikolov-Segal as it is easily derived from the results in [48], although not explicitly stated there in this form. The derivation is included for clarity.)

THEOREM 5.21 (Nikolov-Segal). Let $P$ be a topologically finitely generated profinite group having finitely many isomorphism types of composition factors. Then any dense normal subgroup of $P$ contains the derived group $[P, P]$, which is closed in $P$.

Proof. Following [48], we denote by $P_{0}$ the intersection of all open normal subgroups $T$ of $P$ such that there is a nonabelian finite simple group $S$ for which $P / T$ is isomorphic to some subgroup of $\operatorname{Aut}(S)$ containing $\operatorname{Inn}(S)$. The hypothesis that $P$ has finitely many types of composition factors then imposes a bound on $|P: T|$ for such open subgroups $T$. We recall [53, Proposition 2.5.1] that a topologically finitely generated profinite group has only finitely many open subgroups of a given index; thus $P_{0}$ is open in $P$.

Let now $N$ be a dense normal subgroup of $P$. We proceed as in the discussion preceding [48, Corollary 1.8]. Since $N$ is dense and $P_{0}$ is open in $P$, the image of $N$ in $P / P_{0}$ is onto, and the image of $N$ in $P / \overline{[P, P]}$ is dense. Using the fact that $P$ is topologically finitely generated, we may then find a finite set of elements $y_{1}, \ldots, y_{r} \in N$ such that $P_{0} \overline{\left\langle y_{1}, \ldots, y_{r}\right\rangle}=\overline{[P, P]\left\langle y_{1}, \ldots, y_{r}\right\rangle}=P$. Invoking [48, Theorem 1.7], we then infer that $\left[P_{0}, P\right]$ is entirely contained in $N$. Therefore, $[P, P]=\left[N P_{0}, P\right] \leqslant N\left[P_{0}, P\right] N \leqslant N$, hence $[P, P]$ is entirely contained in $N$, as desired.

The fact that $[P, P]$ is closed follows from [48, Corollary 5.9].

We recall from Proposition 4.6 that if $G$ is a compactly generated t.d.l.c. group whose only compact normal subgroup is the trivial one, then every compact open subgroup of $G$ has finitely many isomorphism types of composition factors. The hypotheses of Theorem 5.21 are thus naturally satisfied in that context. We point out the following consequence, part of which was first noticed in a conversation with Nikolay Nikolov several years ago (see the unpublished preprint [47, Proposition 4]). 
COROLlaRY 5.22. Let $G$ be a t.d.l.c.s.c. group. Let $U$ be a compact open subgroup of $G$, and suppose that $U$ is topologically finitely generated with finitely many types of composition factors.

(i) Let $D$ be a dense normal subgroup of $G$. Then $D \geqslant[U, U]$.

(ii) Let $L$ be a commensurated compact subgroup of $G$, and suppose that $D=$ $\langle\langle L\rangle$ is dense in $G$ and $L$ is normal in $U$. Then $L \cap[U, U]$ has finite index in $[U, U]$ and $D \geqslant[G, G]$.

(iii) Suppose $G$ is topologically simple. If $G$ is not compactly generated, assume also that $U$ is nonabelian. Then $[G, G]=\langle\langle[U, U]\rangle$ is the unique smallest dense normal subgroup of $G$. If $[U, U]$ has finite index in $U$ or $G=[G, G]$, then $G$ has no proper dense normal subgroups.

Proof. Let now $D$ be a dense normal subgroup of $G$. Then $D \cap U$ is a dense normal subgroup of $U$, so part (i) follows by Theorem 5.21.

Now suppose that $D=\langle\langle L\rangle$, where $L$ is a commensurated compact subgroup of $G$ that is normal in $U$. By part (i), we see that $[U, U] \leqslant D$. Moreover, $D$ is $L$-meagre by Lemma 3.14, so [U,U] is $L$-meagre. Since $[U, U]$ is closed by Theorem 5.21 , in fact $[U, U]$ is contained in the union of finitely many cosets of $L$.

In particular, we see that $U / L$ is virtually abelian, so $D \geqslant[G, G]$ by Proposition 5.17, finishing the proof of (ii).

Suppose $G$ is topologically simple. If $G$ is compactly generated, then $U$ cannot be abelian by Theorem 5.3. Thus the locally normal subgroup $L=[U, U]$ is nontrivial. By part (i), the group $D=\langle\langle L\rangle\rangle$ is contained in every dense normal subgroup of $G$; moreover, since $G$ is topologically simple, $D$ is dense in $G$, so it is the unique smallest dense normal subgroup of $G$. By part (ii), we have $D \geqslant[G$, $G]$; clearly $D \leqslant[G, G]$, so in fact $D=[G, G]$. If $G=[G, G]$ then $D=G$; alternatively, if $[U, U]$ has finite index in $U$, then $D$ is open and dense in $G$, so $D=G$. In either case, the minimality of $D$ ensures that there are no proper dense normal subgroups of $G$, so $G$ is abstractly simple.

5.5. Commensurated open subgroups. We have seen in Proposition 5.20 how if there is a nontrivial, nonopen commensurated compact locally normal subgroup in a group $G \in \mathscr{S}$, it would give rise to another group $S \in \mathscr{S}$ embedded in $G$ as a dense normal subgroup. The intersection of $S$ with the compact open subgroups of $G$ would then be commensurated noncompact open subgroups of $S$. Using Proposition 5.17, we can constrain the possible structure of these commensurated open subgroups, leading to a proof of Theorem C. Recall that 
an $\overline{\mathrm{FC}}$-group is a topological group in which every conjugacy class has compact closure.

LEMMA 5.23. Let $G$ be a t.d.l.c.s.c. group, let $U<G$ be a compact open subgroup and $L$ be a nontrivial closed normal subgroup of $U$ which is commensurated by $G$. Let $D=\langle\langle L\rangle$ and set $\alpha=[L]$; suppose $D$ is dense in $G$. Then, with respect to the topology $\mathcal{T}_{(\alpha)}$, the subgroup $D \cap U<D$ is open, $\overline{\mathrm{FC}}$, and commensurated by $D$.

Proof. It is clear that $D \cap U$ is $\mathcal{T}_{(\alpha)}$-open. Since $G$ commensurates $U$, it follows that $D$ commensurates $D \cap U$. We observe that if $\pi$ is the quotient map given in Proposition 5.17, then the construction of $\pi$ given in the proof ensures that the quotient $(D \cap U) / L$ is isomorphic to $\operatorname{Ker}(\pi)$ as an abstract group. In particular, by Proposition 5.17(3), $(D \cap U) / L$ is a group all of whose conjugacy classes are finite. Since $L$ is compact, this implies that $D \cap U$ is indeed $\overline{\mathrm{FC}}$ with respect to $\mathcal{T}_{(\alpha)}$.

We now show that, conversely, an open commensurated $\overline{\mathrm{FC}}$-subgroup of a group $G \in \mathscr{S}$ naturally yields another group in $\mathscr{S}$ that admits nontrivial fixed points in its structure lattice. In order to do so, we need a few basic facts on $\overline{\mathrm{FC}}$ groups, due to Ušakov. We recall that a subgroup of a locally compact group is called locally elliptic if it is the directed union of its compact subgroups.

Proposition 5.24. Let $G$ be a locally compact $\overline{\mathrm{FC}}$-group.

(i) $G$ is the directed union of its compactly generated closed normal subgroups.

(ii) The set of compact subgroups of $G$ is a directed set (for the relation of inclusion), so that its union $R$ is a closed characteristic locally elliptic subgroup of $G$ such that $G / R$ is torsion-free abelian. In particular $G$ is amenable.

(iii) If $G$ is compactly generated, then $R$ is compact.

Proof. It is clear from the definition that every element is contained in a compactly generated closed normal subgroup. The fact that the collection of all those subgroups is directed (for the relation of inclusion) is straightforward. This proves (i). The assertions (ii) and (iii) are proved in [65]. An alternative (and easier to find) reference is [66].

Lemma 5.25. Let $G \in \mathscr{S}$. Let $O<G$ be an open $\overline{\mathrm{FC}}$-subgroup which is commensurated by $G$. Then there exist a group $H \in \mathscr{S}$, a continuous injective 
homomorphism with dense image $\varphi: G \rightarrow H$, and a nontrivial commensurated compact locally normal subgroup $M<G$ such that $\overline{\varphi(O)}$ is a compact open subgroup of $H$ and $\varphi(M)$ is a commensurated compact locally normal subgroup of $H$.

Proof. Since $O$ is nondiscrete and closed in $G$, it contains a nontrivial element which generates a cyclic subgroup with compact closure. By Proposition 5.24, this compact subgroup is contained in a closed, compactly generated normal subgroup of $O$, say $P$. By construction, the largest compact subgroup of $P$ afforded by Proposition 5.24, say $R$, is nontrivial, and normal in $O$.

By hypothesis $(G, O)$ is a Hecke pair. By Theorem 3.2 and Proposition 3.6, there exists a group $H \in \mathscr{S}$ and a homomorphism $\varphi: G \rightarrow H$ with dense image such that $V=\overline{\varphi(O)}$ is a compact open subgroup of $H$. Since $O$ is open, the homomorphism $\varphi$ is continuous. Since moreover $H$ is nontrivial and $G$ is topologically simple, it follows that $\varphi$ is injective.

Now $K=\varphi(R)$ is a nontrivial compact subgroup of $V$ normalized by $\varphi(O)$. Therefore, $K$ is a nontrivial compact locally normal subgroup of $H$. Since $\varphi(G)$ is dense in $H$, it acts transitively on the conjugacy class of $K$. In particular $\varphi(G)$ contains the normal closure $\langle\langle K\rangle\rangle$ of $K$ in $H$. By Lemma 5.1, there is a finite set $\left\{K_{1}, \ldots, K_{n}\right\}$ of conjugates of $K$ in $H$ and for each $i$, an open subgroup $L_{i} \leqslant K_{i}$ such that the product $L=L_{1} \ldots L_{n}$ is a closed normal subgroup of $V$ which is commensurated by $H$. Since $L$ is contained in $\langle\langle K\rangle$, it is also contained in $\varphi(G)$. Let $M=\varphi^{-1}(L)$. Then $M$ is indeed compact in $G$ (because it is a finite product of compact subgroups of $G$ ). Since $L$ is normal in $V$ and commensurated by $H$, it follows that $M$ is normalized by $O$ and commensurated by $G$, so that $M$ is indeed a commensurated compact locally normal subgroup of $G$.

We can now prove Theorem C.

Proof of Theorem $C$. Assume that (i) holds and that $G \in \mathscr{S}$ possesses an open commensurated $\overline{\mathrm{FC}}$-subgroup $O$. We apply Lemma 5.25, which affords a group $H \in \mathscr{S}$, a dense embedding $\varphi: G \rightarrow H$ and a nontrivial commensurated compact locally normal subgroup $M<G$ such that $\varphi(M)$ is a commensurated compact locally normal subgroup of $H$. By (i) the group $\varphi(M)$ must be open in $H$, so that $\varphi(O)$ is open with compact closure. Therefore, $\varphi(O)$ is compact. If $U<$ $O$ is a compact open subgroup of $O$, it has countable index, so that $\varphi(U)$ is a closed subgroup of countable index in the compact group $\varphi(O)$. That index must therefore be finite. Since $\varphi$ is injective, we infer that $U$ has finite index in $O$, so that $O$ is indeed compact. Since every compact open subgroup of $G \in \mathscr{S}$ is a commensurated $\overline{\mathrm{FC}}$-subgroup of $G$, we infer that (i) implies (iii).

That (iii) implies (ii) is clear. 
Assume that (ii) holds and let $G \in \mathscr{S}$ with an infinite commensurated compact subgroup. By Lemma 2.4, the commensurability class of that compact subgroup has a closed locally normal representative $L$. By Proposition 5.20, there is an infinite commensurated compact locally normal subgroup $M \leqslant L$ whose abstract normal closure $S=\langle\langle M\rangle\rangle$ in $G$, endowed with the topology $\mathcal{T}_{(\beta)}$ with $\beta=[M]$, belongs to the class $\mathscr{S}$. Let $U<G$ be a compact open subgroup containing $M$ as a normal subgroup. By Lemma 5.23, the subgroup $S \cap U$ is a commensurated subgroup of $S$ which is open and $\overline{\mathrm{FC}}$ with respect to the topology $\mathcal{T}_{(\beta)}$. It then follows by applying (ii) to the group $S \in \mathscr{S}$ that $S \cap U$ is $\mathcal{T}_{(\beta)}$-compact. Since $M \leqslant S \cap U$ is $\mathcal{T}_{(\beta)}$-open by definition of the latter topology, it follows that [ $S \cap U$ : $M]$ is finite. Since $S \cap U$ is dense in $U$, it follows that $M$ has finite index in $U$, and so $M$ is open in $G$. Thus (ii) implies (i), completing the proof that (i), (ii) and (iii) are equivalent.

It remains to show the equivalence of (i) and (iv). Suppose (i) holds and let $G$ be a compactly generated t.d.l.c. group with distinct closed normal subgroups $N_{1}$ and $N_{2}$ that are maximal among proper closed normal subgroups of $G$. Note that $N_{1} N_{2}$ is a normal subgroup of $G$; by the maximality of $N_{1}$ and $N_{2}$, we see that $N_{1} N_{2}$ is dense in $G$. If $N_{1} N_{2}$ is open in $G$, then it is also closed and hence $N_{1} N_{2}=G$. Thus to show $N_{1} N_{2}=G$, we need only show that $N_{1} N_{2}$ is open. The quotients $G / N_{1}$ and $G / N_{2}$ are compactly generated and topologically simple; we may assume that $N_{1}$ and $N_{2}$ are not open, and thus $G / N_{1}, G / N_{2} \in \mathscr{S}$. We now have a continuous injective homomorphism with dense image from $N_{1} /\left(N_{1} \cap\right.$ $\left.N_{2}\right)$ to $S_{2}:=G / N_{2}$ given by $g\left(N_{1} \cap N_{2}\right) \mapsto g N_{2}$. Since $N_{1}$ is normal in $G$, any compact open subgroup $K$ of $N_{1}$ is commensurated in $G$, and thus $K_{2}:=$ $K N_{2} / N_{2}$ is commensurated in $S_{2}$. Since QZ $\left(S_{2}\right)=\{1\}$ by Theorem 5.3, $N_{1} N_{2} / N_{2}$ is nondiscrete and thus $K_{2}$ is infinite. By (i), it follows that $K_{2}$ is open in $S_{2}$, so $N_{1} N_{2}$ is open in $G$. Hence $G=N_{1} N_{2}$, proving (iv).

On the other hand, suppose now that (i) does not hold: that is, there is $G \in \mathscr{S}$ and $L \leqslant G$ such that $L$ is infinite, commensurated and not open in $G$. We now construct a counterexample to (iv). By Lemma 2.4 we may assume that $L$ is locally normal in $G$. Let $D=\langle\langle L\rangle\rangle$ be the abstract normal closure of $L$ in $G$. Applying Proposition 5.20, there is an infinite commensurated locally normal subgroup $M$ of $G$ such that $M \leqslant L$, the group $S:=\langle\langle M\rangle\rangle$ is compactly generated and topologically simple in the $\mathcal{T}_{[M]}$-topology and $[D, D] \leqslant S \leqslant D$. By Lemma 3.14, $D$ and hence $S$ is a proper subgroup of $G$. Equip $S$ with the $\mathcal{T}_{[M]^{-}}$ topology and form the semidirect product $H:=S \rtimes G$ with the product topology as in the discussion before Proposition 5.17. Then $H$ is a compactly generated t.d.l.c. group with (at least) two distinct surjective continuous homomorphisms $\pi_{1}, \pi_{2}: H \rightarrow G$, given by $\phi_{1}(s, g)=g$ and $\phi_{2}(s, g)=s g$. The kernels $N_{1}:=\operatorname{Ker}\left(\pi_{1}\right)=S \rtimes\{1\}$ and $N_{2}:=\operatorname{Ker}\left(\pi_{2}\right)=\left\{\left(s^{-1}, s\right) \mid s \in S\right\}$ are then 
distinct subgroups of $H$ that are maximal among proper closed normal subgroups of $H$. However, $N_{1} N_{2}=S \rtimes S \neq S \rtimes G$, so $N_{1} N_{2}$ is a proper subgroup of $H$. We have our desired counterexample to (iv), completing the proof that (i) and (iv) are equivalent.

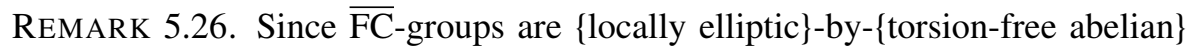
by Proposition 5.24, one may wonder whether an alternative version of assertion (ii) in Theorem $\mathrm{C}$ could be true: could it be that for all $G \in \mathscr{S}$, every commensurated locally elliptic open subgroup of $G$ is compact? The answer turns out to be negative in this case. Indeed, there are examples of groups $G \in \mathscr{S}$ which act continuously but nonproperly by automorphisms on regular locally finite trees (see [37]). The stabilizer of a vertex in $G$ is then an open, but noncompact, locally elliptic subgroup, which is commensurated by $G$.

\section{Dynamics of conjugation of locally normal subgroups}

The conjugation action of a t.d.l.c. group $G$ on its closed locally normal subgroups has an interesting dynamical property under the conditions that $G$ is compactly generated and locally $\mathrm{C}$-stable, has an identity neighbourhood containing no nontrivial compact normal subgroups, and acts faithfully on the Boolean algebra $\mathcal{L C}(G)$. We begin this section with the relevant definitions and results for actions of groups on general Boolean algebras, $\mathcal{A}$, and profinite spaces, $\Omega$, before proceeding the case when $\mathcal{A}$ is $\mathcal{L C}(G)$ and $\Omega$ is the corresponding Stone space.

6.1. Rigid stabilizers. Let $\mathcal{A}$ be a Boolean algebra. Then by the Stone representation theorem, $\mathcal{A}$ defines a profinite space (that is, a compact zerodimensional space), the Stone space $\mathfrak{S}(\mathcal{A})$ of $\mathcal{A}$, whose points are the ultrafilters of $\mathcal{A}$ and the topology is generated by subsets of the form $\{\mathfrak{p} \in \mathfrak{S}(\mathcal{A}) \mid \alpha \in \mathfrak{p}\}$ with $\alpha \in \mathcal{A}$. Conversely, given a profinite space $\mathfrak{X}$, the set $\mathcal{A}(\mathfrak{X})$ of clopen subsets of $\mathfrak{X}$ form a Boolean algebra. This correspondence produces a natural isomorphism between $\operatorname{Aut}(\mathcal{A})$ and $\operatorname{Aut}(\mathfrak{S}(\mathcal{A}))$. In practice we often find it convenient to abuse notation and treat elements of $\mathcal{A}$ as subsets of $\mathfrak{S}(\mathcal{A})$, identifying $\alpha \in \mathcal{A}$ with the clopen set

$$
\mathfrak{S}(\alpha):=\{\mathfrak{p} \in \mathfrak{S}(\mathcal{A}) \mid \alpha \in \mathfrak{p}\} \in \mathcal{A}(\mathfrak{S}(\mathcal{A})) .
$$

The expressions ' $\alpha \in \mathfrak{p}$ ' and ' $\mathfrak{p} \in \alpha$ ' for $\alpha \in \mathcal{A}$ and $\mathfrak{p} \in \mathfrak{S}(\mathcal{A})$ can therefore be taken to be synonymous.

Given a group $G$ acting on a set $X$ and $x \in X$, we write $G_{x}$ for the stabilizer $\{g \in G \mid g x=x\}$. 
Definition 6.1. Let $G$ be a topological group acting on a set $\mathcal{A}$. Say that the action of $G$ on $\mathcal{A}$ is smooth if every point stabilizer is open. In particular, for every compact open subgroup $U$ of $G$, the orbits of $U$ on $\mathcal{A}$ are all finite.

The following lemma shows the relevance of smooth actions for t.d.l.c. groups:

LEMMA 6.2 [21, Lemma 5.11]. Let $G$ be a t.d.l.c. group, let $\mathfrak{X}$ be a profinite space and $\mathcal{A}$ a Boolean algebra.

(i) If $G$ acts on $\mathfrak{X}$ by homeomorphisms, and if the $G$-action is continuous with respect to the topology of uniform convergence, then the corresponding $G$ action on the Boolean algebra of clopen subsets of $\mathfrak{X}$ is smooth.

(ii) If $G$ acts on $\mathcal{A}$ by automorphisms, and if the G-action is smooth, then the corresponding $G$-action on the Stone space $\mathfrak{S}(\mathcal{A})$ is continuous with respect to the topology of uniform convergence. In particular, the action map $(g, x) \mapsto g x$ is a continuous map from $G \times \mathfrak{S}(\mathcal{A})$ to $\mathfrak{S}(\mathcal{A})$.

DEFINITION 6.3. Let $G$ be a group acting on a set $Z$. Given a subset $v \subseteq Z$, the rigid stabilizer of $v$ is the subgroup

$$
\operatorname{rist}_{G}(v):=\{g \in G \mid g z=z \forall z \in Z \backslash v\} .
$$

Notice that if $\mathcal{A}$ is a subalgebra of $\mathcal{L C}(G)$ or of $\mathcal{L D}(G)$, then $\mathrm{QC}_{G}(\alpha) \leqslant$ rist $_{G}\left(\alpha^{\perp}\right)$ for all $\alpha \in \mathcal{A}$. We emphasize that, in the notation $\operatorname{rist}_{G}\left(\alpha^{\perp}\right)$, we have relied on the (abusive) convention explained above to identify an element of a Boolean algebra with the corresponding subset of the associated Stone space under the Stone correspondence. In particular, every nonzero element of $\mathcal{A}$ has an infinite rigid stabilizer in $G$. Moreover $\operatorname{rist}_{G}(v)$ is a closed subgroup of $G$ for any $v \subseteq \mathfrak{S}(\mathcal{A})$, since $\operatorname{rist}_{G}(v)$ can be expressed as an intersection of point stabilizers, each of which is closed by the fact that the action of $G$ is continuous.

DEFINITION 6.4. Let $G$ be a t.d.l.c. group acting on a Boolean algebra $\mathcal{A}$ with kernel $K$. Say that the action is weakly decomposable if it is smooth and the quotient group $\operatorname{rist}_{G}(\alpha) / K$ is nontrivial for every $\alpha \in \mathcal{A} \backslash\{0\}$. Say the action is locally weakly decomposable if moreover $\operatorname{rist}_{G}(\alpha) / K$ is nondiscrete for every $\alpha \in \mathcal{A} \backslash\{0\}$.

When $G$ acts faithfully and has trivial quasicentre, which will often be the case, then a faithful action is weakly decomposable if and only if it is locally weakly decomposable: $\operatorname{rist}_{G}(\alpha)$ is a normal subgroup of $\operatorname{Stab}_{G}(\alpha)$, which is open in $G$, 
so that rist ${ }_{G}(\alpha)$ would be in the quasicentre of $G$ if it were discrete. Under these conditions, the weakly decomposable condition also ensures that $\operatorname{rist}_{G}(\alpha)$ is not locally equivalent to $\operatorname{rist}_{G}(\beta)$ for any two distinct elements $\alpha, \beta \in \mathcal{A}$.

In view of Lemma 6.2 , the definition of weak decomposability of the action of $G$ on a Boolean algebra given in Definition 6.4 is equivalent with the definition of weak decomposability of the action of $G$ on a profinite space given before Theorem J.

Proposition 6.5. Let $G$ be a t.d.l.c. group, $\mathfrak{X}$ be a profinite space and $\mathcal{A}$ a Boolean algebra.

(i) A continuous action of $G$ on $\mathfrak{X}$ by homeomorphisms is (locally) weakly decomposable if and only if the corresponding $G$-action on the Boolean algebra of clopen subsets of $\mathfrak{X}$ is (locally) weakly decomposable.

(ii) A smooth action of $G$ on $\mathcal{A}$ by automorphisms is (locally) weakly decomposable if and only if the corresponding $G$-action on the Stone space $\mathfrak{S}(\mathcal{A})$ is (locally) weakly decomposable.

We recall the following result from [21], which shows that locally weakly decomposable actions appear naturally in the context of the centralizer or local decomposition lattices.

Proposition 6.6 (See [21, Proposition 5.16]). Let $G$ be a locally $C$-stable t.d.l.c. group such that $\mathrm{QZ}(G)=\{1\}$ and let $\mathcal{A}$ be a $G$-invariant subalgebra of $\mathcal{L C}(G)$. Then the $G$-action on $\mathcal{A}$ is locally weakly decomposable as soon as it is faithful. More precisely, if the $G$-action is faithful, then for each $\alpha \in \mathcal{A}$ we have rist $_{G}(\alpha)=\mathrm{QC}_{G}\left(\mathrm{QC}_{G}((\alpha))=\mathrm{C}_{G}\left(\mathrm{QC}_{G}(\alpha)\right)=\mathrm{QC}_{G}\left(\alpha^{\perp}\right) \quad\right.$ and $\quad \alpha=\left[\operatorname{rist}_{G}(\alpha)\right]$, where $\mathrm{QC}_{G}(\alpha)$ denotes the quasicentralizer of any compact representative of $\alpha$.

We close this subsection with an auxiliary assertion which will be used several times in the sequel. We use the following terminology. Let $\mathcal{A}$ be a Boolean algebra. A partition $\mathcal{P}$ of $\alpha \in \mathcal{A}$ is a finite subset of $\mathcal{A}$ such that the join of $\mathcal{P}$ is $\alpha$ and the meet of any two distinct elements of $\mathcal{P}$ is 0 ; a partition of $\mathcal{A}$ is just a partition of $\infty$ in $\mathcal{A}$. A partition $\mathcal{P}_{1}$ is called finer than (or a refinement of) a partition $\mathcal{P}_{2}$, written $\mathcal{P}_{1} \leqslant \mathcal{P}_{2}$, if for every $\alpha_{1} \in \mathcal{P}_{1}$, there exists $\alpha_{2} \in \mathcal{P}_{2}$ with $\alpha_{1} \leqslant \alpha_{2}$.

LEMMA 6.7. Let $\mathcal{A}$ be a Boolean algebra and $G$ be a t.d.l.c. group endowed with a smooth faithful action on $\mathcal{A}$ by automorphisms. For all compact open subgroups $V \leqslant U \leqslant G$, there exists a $U$-invariant partition $\mathcal{C}$ of $\mathcal{A}$ such that $\bigcap_{\gamma \in \mathcal{C}} U_{\gamma} \leqslant V$. 
Proof. Let $\mathcal{T}$ be the collection of all $U$-invariant partitions of $\mathcal{A}$. Since the $G$ action is smooth, every $U$-orbit on $\mathcal{A}$ is finite. In particular, any finite set of partitions of $\mathcal{A}$ has a common refinement that is $U$-invariant. In particular $\mathcal{T}$ is a directed set and $\bigcup \mathcal{T}$ generates $\mathcal{A}$.

Suppose now for a contradiction that for each $\mathcal{C} \in \mathcal{T}$, there exists $z_{\mathcal{C}} \in \bigcap_{\gamma \in \mathcal{C}} U_{\gamma}$ with $z_{\mathcal{C}} \notin V$. Since $U$ is compact and $V$ is open, the net $\left(z_{\mathcal{C}}\right)_{\mathcal{C} \in \mathcal{T}}$ has a subnet $\left(z_{\mathcal{C}}\right)_{\mathcal{C} \in \widetilde{\mathcal{T}}}$ converging to some $z \in U \backslash V$, where $\widetilde{\mathcal{T}}$ is a final subset of the directed set $\mathcal{T}$.

We now consider an arbitrary element $\alpha \in \mathcal{A}$. By the first paragraph of the proof above, there exists $\mathcal{P} \in \widetilde{\mathcal{T}}$ which contains a partition of $\alpha$. Therefore, $\mathcal{C}$ contains a partition of $\alpha$ for every partition $\mathcal{C} \leqslant \mathcal{P}$. In particular, we have $z_{\mathcal{C}} \in \bigcap_{\gamma \in \mathcal{C}} U_{\gamma} \leqslant$ $U_{\alpha}$ for all $\mathcal{C} \in \mathcal{T}$ with $\mathcal{C} \leqslant \mathcal{P}$. Therefore, $z=\lim _{\mathcal{C} \in \widetilde{\mathcal{T}}} z_{\mathcal{C}}$ is contained in $U_{\alpha}$. It follows that $z$ is an element of $U \backslash V$ acting trivially on $\mathcal{A}$. This contradicts the hypothesis that the $G$-action on $\mathcal{A}$ is faithful.

6.2. A simplicity criterion. We now give the proof of the simplicity criterion from Proposition I. It relies on the following subsidiary fact.

Lemma 6.8. Let $A, N$ be subgroups of a group $G$. Assume that $N$ is normal, and contains an element $t \in N$ such that $\left[A, t A t^{-1}\right]=\{1\}$. Then $N$ contains $[A, A]$.

Proof. Let $a, b, c \in A$. Since $a$ and $b$ commute with $t c t^{-1}$, we have $[a, b]=$ $\left[a, b t c t^{-1}\right]$. Setting $c=b^{-1}$, we infer that $[a, b]=[a,[b, t]]$, which belongs to $N$ since $N$ is normal.

Proof of Proposition I. We must show that the monolith of $G$ (viewed as a discrete group) is nontrivial.

We first show that the rigid stabilizer of any nonempty open set $U \subseteq X$ is nonabelian. Since the $G$-action is microsupported, we already know that the rigid stabilizer $\operatorname{rist}_{G}(U)$ is nontrivial. Pick $g \in \operatorname{rist}_{G}(U)$ and $x \in X$ with $g x \neq x$. Since $X$ is Hausdorff, there exists a neighbourhood $V$ of $x$ contained in $U$ such that $g V \cap V=\varnothing$. The rigid stabilizer $\operatorname{rist}_{G}(V)$ is nontrivial and contained in $\operatorname{rist}_{G}(U)$. Moreover the respective supports of $\operatorname{rist}_{G}(V)$ and $\operatorname{grist}_{G}(V) g^{-1}=\operatorname{rist}_{G}(g V)$ are disjoint, so that the commutator $\left[g, \operatorname{rist}_{G}(V)\right]$ is nontrivial. In particular rist ${ }_{G}(U)$ is nonabelian.

Let now $O$ be a nonempty open compressible subset of $X$. The previous paragraph implies that $\operatorname{rist}_{G}(O)$ is nonabelian. Let $N$ be any nontrivial normal subgroup of $G$. Then we may find an element $t \in N$ and a nonempty open subset $V$ of $X$ such that $t V \cap V=\varnothing$. Since $O$ is compressible there exists $g \in G$ such that $Q=g O \subset V$. In particular we have $\left[\operatorname{rist}_{G}(Q), \operatorname{trist}_{G}(Q) t^{-1}\right]=1$. 
By Lemma 6.8, the group $N$ contains $\left[\operatorname{rist}_{G}(Q), \operatorname{rist}_{G}(Q)\right]$, hence also [rist $\operatorname{rin}_{G}(O)$, rist $\left._{G}(O)\right]=g^{-1}\left[\operatorname{rist}_{G}(Q)\right.$, rist $\left._{G}(Q)\right] g$. Therefore, the monolith $\operatorname{Mon}(G)$ contains $\left[\operatorname{rist}_{G}(O)\right.$, rist $\left._{G}(O)\right]$ and is thus nontrivial.

What we have just established implies moreover that the $\operatorname{Mon}(G)$-action on $X$ is microsupported. If we assume in addition that $\operatorname{Mon}(G)$ has a nonempty compressible open set, then the first part of the proof implies that $\operatorname{Mon}(\operatorname{Mon}(G))$ is nontrivial. Since the monolith is a characteristic subgroup, it follows that $\operatorname{Mon}(\operatorname{Mon}(G))$ is a nontrivial normal subgroup of $G$, which must thus contain $\operatorname{Mon}(G)$. In other words we have $\operatorname{Mon}(\operatorname{Mon}(G))=\operatorname{Mon}(G)$, which means precisely that $\operatorname{Mon}(G)$ is simple.

6.3. Minorizing actions on Boolean algebras. Recall, for $\Omega$ a topological $G$ space, the following definition from the introduction. Let $\omega \in \Omega$ a subset $F \subset \Omega$ is compressible to the point $\omega$ if there is a basis of neighbourhoods $\left(V_{i}\right)$ of $\omega \in \Omega$ such that for each $V_{i}$, there is $g \in G$ with $g F \subset V_{i}$. We say that $F$ is compressible if for every nonempty open set $O$ in $\Omega$, there is $g \in G$ with $g F \subset O$. Note that $F$ is compressible if and only if it is compressible to every point. Moreover, if $F$ is compressible to the point $\omega$ and if the $G$-orbit of $\omega$ is dense, then $F$ is compressible.

In this subsection we introduce the closely related concept of a minorizing action, which will play a critical role in obtaining structural properties of groups in $\mathscr{S}$ with nontrivial centralizer lattice.

Definition 6.9. Let $\mathcal{A}$ be a poset with least element 0 and let $G$ be a group acting on $\mathcal{A}$ by automorphisms. Say a subset $\mathcal{A}^{\prime}$ of $\mathcal{A}$ is minorizing if for all $\alpha \in \mathcal{A} \backslash\{0\}$ there is some $\beta \in \mathcal{A}^{\prime}$ such that $0<\beta<\alpha$. (We emphasize that the inequality is understood to be strict. In particular that if $\mathcal{A}$ has a minorizing set, then it cannot have any atoms.) Say $\mathcal{A}^{\prime}$ is minorizing under the action of $G$ if $\bigcup_{\alpha \in \mathcal{A}^{\prime}} G \alpha$ is minorizing. We say the action of $G$ on $\mathcal{A}$ is minorizing (of degree $d \in \mathbf{N}$ ) if there is a finite minorizing set for the action (and the minimum number of elements in such a set is $d$ ).

The following lemma shows the relationship between minorizing elements of the Boolean algebra and compressible subsets of the Stone space.

LEMMA 6.10. Let $G$ be a group of homeomorphisms of the profinite space $\Omega$, let $\alpha$ be a nonempty clopen subset of $\Omega$ and let $\mathcal{A}$ be the Boolean algebra of clopen subsets of $\Omega$. Let $X$ be the set (possibly empty) of points $\omega \in \Omega$ such that $\alpha$ is $G$ compressible to $\omega$. Then $X$ is a closed $G$-invariant set; moreover, $\alpha$ is minorizing for the action of $G$ on $\mathcal{A}$ if and only if $X=\Omega$. 
Proof. Suppose $\omega \in X$ and let $\left(V_{i}\right)$ be a base of neighbourhoods of $\omega$. For each $i$, there is some $g_{i} \in G$ such that $g_{i} \alpha \subseteq V_{i}$. Now let $g \in G$. Since $g$ is a homeomorphism, the net $\left(g V_{i}\right)$ forms a base of neighbourhoods of $g \omega$, and clearly we have $g g_{i} \alpha \subseteq g V_{i}$. Thus $\alpha$ is $G$-compressible to $g \omega$, so $X$ is $G$-invariant.

Suppose $\omega \notin X$. Then by the definition of $X$, there is some neighbourhood $O$ of $\omega$ that does not contain any $G$-image of $\alpha$. We see that in fact $\alpha$ is not compressible to $\omega^{\prime}$ for any $\omega^{\prime} \in O$. Thus $\Omega \backslash X$ is open, in other words $X$ is closed.

Suppose $X=\Omega$ and let $\beta \in \mathcal{A} \backslash\{0\}$. Then $\beta$ is a nonempty open subset of $\Omega$, so $\beta$ is a neighbourhood of some point $\omega \in \beta$. Hence there exists $g \in G$ such that $g \alpha \subset \beta$. Hence the $G$-orbit of $\alpha$ is minorizing in $\mathcal{A}$.

Conversely, suppose that $G \alpha$ is minorizing in $\mathcal{A}$. Since $\Omega$ is a profinite space, for every point $\omega \in \Omega$ there is a base of neighbourhoods of $\omega$ consisting of clopen sets. For every such clopen set $\beta$, there exists $g \in G$ such that $g \alpha<\beta$. Thus $\alpha$ is $G$-compressible to every point in $\Omega$.

In the case of minorizing actions on Boolean algebras, we can obtain a canonical structure in the Stone space that accounts for the degree of the minorizing action.

LEMMA 6.11. Let $G$ be a group with a minorizing action of degree $d$ on the Boolean algebra $\mathcal{A}$.

(i) Let $\mathcal{U}$ be the set of minimal nonempty $G$-invariant open subsets of $\mathfrak{S}(\mathcal{A})$. Then $|\mathcal{U}|=d$, and every $G$-invariant subset of $\mathfrak{S}(\mathcal{A})$ with a nonempty interior contains some element of $\mathcal{U}$.

(ii) If $d=1$, then the union of the dense orbits of $G$ on $\mathfrak{S}(\mathcal{A})$ is a nonempty (hence dense) open set. If $d>1$, there are no dense orbits of $G$ on $\mathfrak{S}(\mathcal{A})$.

Proof. (i) Let $G$ be a group with a minorizing action on the Boolean algebra $\mathcal{A}$ and let $\left\{\alpha_{1}, \ldots, \alpha_{d}\right\}$ be a minorizing set for the action of minimal size. For each $1 \leqslant i \leqslant d$, let $v_{i}$ be the union of all $G$-translates of $\alpha_{i}$ in $\mathfrak{S}(\mathcal{A})$. We claim that $\mathcal{U}=\left\{v_{1}, \ldots, v_{d}\right\}$.

Suppose that $B \subseteq \mathfrak{S}(\mathcal{A})$ has a nonempty interior. Then there is $\beta \in \mathcal{A}$ contained in $B$ and the minorizing property implies that there are $i \in\{1, \ldots, d\}$ and $g \in G$ such that $g \alpha_{i} \leqslant \beta$. If $B$ is also $G$-invariant, it hence follows that $v_{i}$ is contained in $B$. Once it is shown that the sets $\left\{v_{1}, \ldots, v_{d}\right\}$ are pairwise disjoint, it will follow that every minimal open $G$-invariant subset of $\mathfrak{S}(\mathcal{A})$ is one of the sets $v_{i}$, and conversely. Let us suppose for a contradiction that $v_{i} \cap v_{j} \neq \varnothing$. Then, by definition of $v_{i}$ and $v_{j}$, there are $g_{i}, g_{j} \in G$ such that $g_{i} \alpha_{i} \cap g_{j} \alpha_{j} \neq \varnothing$. Since $g_{i} \alpha_{i} \cap$ $g_{j} \alpha_{j}$ is open, there are $k \in\{1, \ldots, d\}$ and $g \in G$ such that $g \alpha_{k} \subseteq g_{i} \alpha_{i} \cap g_{j} \alpha_{j}$. 
Hence the set obtained by removing $\alpha_{i}$ and $\alpha_{j}$ from $\left\{\alpha_{1}, \ldots, \alpha_{d}\right\}$ and including $\alpha_{k}$ is minorizing for the action of $G$. That contradicts minimality of the set unless $i=j=k$. Hence the elements of $\left\{v_{1}, \ldots, v_{d}\right\}$ are pairwise disjoint and this set is equal to $\mathcal{U}$.

(ii) Suppose $d=1$. Then $\mathcal{U}=\left\{v_{1}\right\}$ and for any open set $\alpha$ in $\mathfrak{S}(\mathcal{A})$ and any point $\mathfrak{p} \in v_{1}$, there exists $g \in G$ with $g \mathfrak{p} \in \alpha$ by the definition of $v_{1}$. Therefore, the $G$-orbit of every element of $v_{1}$ is dense. Since $v_{1}$ is open and nonempty, no element outside $v_{1}$ can have a dense orbit.

Suppose now that $d>1$. Then $v_{1}$ and $v_{2}$ are disjoint by the above, and both are nonempty and $G$-invariant. Therefore no element can have a dense orbit.

6.4. Skewering automorphisms of Boolean algebras. The minorizing property ensures the existence of skewering elements, defined as follows.

Definition 6.12. Let $\mathcal{A}$ be a poset and let $g$ be an automorphism of $\mathcal{A}$. Say $g$ is skewering if there is some $\alpha \in \mathcal{A}$ such that $g \alpha<\alpha$. Again, we emphasize that the inequality is understood to be strict.

LEMMA 6.13. Let $\mathcal{A}$ be a poset with least element 0 and let $G$ be a group acting on $\mathcal{A}$, such that the action is minorizing. Let $\mathcal{C}$ be a finite minorizing set under the action of $G$ such that $|\mathcal{C}|$ is minimized. Then for each $\alpha \in \mathcal{C}$ and $0<\beta \leqslant \alpha$, there is $g \in G$ such that $g \alpha<\beta$. In particular, there are elements of $G$ whose action on $\mathcal{A}$ is skewering.

Proof. By definition, there is some $\gamma \in \mathcal{C}$ and $g \in G$ such that $0<g \gamma<\beta$. We must have $\gamma=\alpha$ because $\mathcal{C} \backslash\{\alpha\}$ would be a minorizing set otherwise.

Before the next proposition, let us recall some terminology.

Given an automorphism $f$ of $G$, the contraction group $\operatorname{con}(f)$ of $f$ on $G$ is given by

$$
\operatorname{con}(f)=\left\{u \in G \mid f^{n}(u) \rightarrow 1 \text { as } n \rightarrow+\infty\right\} .
$$

One sees that $\operatorname{con}(f)$ is a subgroup of $G$, although not necessarily a closed subgroup. Given an element $g \in G$, we define $\operatorname{con}(g)$ to be the contraction group of the automorphism induced by left conjugation.

We define the Tits core of a totally disconnected, locally compact group $G$ to be the subgroup

$$
G^{\dagger}:=\overline{\operatorname{con}(g)}|g \in G\rangle .
$$

The nub $\operatorname{nub}(f)$ of an automorphism $f$ of a t.d.l.c. group $G$ is the largest compact $f$-invariant subgroup $N$ of $G$ such that $f$ does not stabilize any proper open subgroup of $N$; see [71] for more details. 
Proposition 6.14. Let $G$ be a t.d.l.c. group acting faithfully on a Boolean algebra $\mathcal{A}$ such that the action is locally weakly decomposable. Let $U$ be a compact open subgroup of $G$. Suppose that there is some $\alpha \in \mathcal{A}$ and $g \in G$ such that $g \alpha<\alpha$; let $\beta=\alpha \backslash g \alpha$.

(i) There is a natural number $n_{0}$ and a closed subset $\kappa$ of $\alpha$ satisfying the following properties: We have $g^{n_{0}} \beta \subset \kappa$, the group $\operatorname{rist}_{U}(\kappa)$ is nontrivial and moreover

$$
g \operatorname{rist}_{U}(\kappa) g^{-1} \times \operatorname{rist}_{U}\left(g^{n_{0}} \beta\right) \leqslant \operatorname{rist}_{U}(\kappa) .
$$

In particular $\left\{g^{n} \operatorname{rist}_{U}(\kappa) g^{-n}\right\}_{n \geqslant 0}$ is a strictly decreasing sequence of compact subgroups of $U$. Moreover $\bigcap_{n \geqslant 0} g^{n} \operatorname{rist}_{U}(\kappa) g^{-n}=1$ and $\operatorname{con}(g)$ contains the group $\operatorname{rist}_{U}(\kappa)$.

(ii) There is a closed subgroup of $G$ of the form

$$
\overline{\left\langle L_{0}, g\right\rangle} \cong \prod_{i \in \mathbf{Z}} L_{i} \rtimes\langle g\rangle,
$$

where $L_{i}=g^{i}$ rist $_{W}(\beta) g^{-i}$ for some compact open subgroup $W$. In particular, $\operatorname{nub}(g)$ contains the direct product $\prod_{i \in \mathbf{Z}} L_{i}$, and hence $\operatorname{con}(g)$ is not closed.

Proof. (i) Let $V=U \cap g^{-1} U g$. The action of $U$ on $\mathcal{A}$ is faithful and smooth. By Lemma 6.7, there is a $U$-invariant partition $\mathcal{C}$ of $\mathcal{A}$ such that $\bigcap_{\gamma \in \mathcal{C}} U_{\gamma} \leqslant V$.

By hypothesis we have $g \alpha<\alpha$. Therefore, for each $\gamma \in \mathcal{C}$ we have either: (a) $g^{n} \alpha \geqslant \gamma$ for all $n \geqslant 0$; or (b) there is $n_{0} \geqslant 0$ such that $g^{n} \alpha \geqslant \gamma$ for all $n \geqslant n_{0}$. Choose a fixed $n_{0}$ sufficiently large that either (a) or (b) holds for every $\gamma \in \mathcal{C}$.

Let $\xi$ be the interior of $\bigcap_{n \in \mathbf{Z}} g^{n} \alpha$ in the Stone space $\mathfrak{S}(\mathcal{A})$. Then the set

$$
\kappa:=g^{n_{0}} \alpha \backslash \xi
$$

is closed. Since $g \xi=\xi$, we have $g \kappa \subset \kappa$. Furthermore $\kappa \backslash g \kappa=g^{n_{0}} \alpha \backslash g^{n_{0}+1} \alpha=$

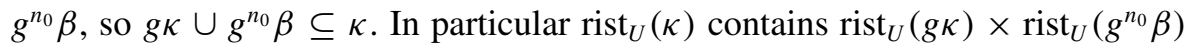
as a subgroup and hence is nontrivial (and even nondiscrete) since the $G$-action on $\mathcal{A}$ is locally weakly decomposable. Moreover, given $\gamma \in \mathcal{C}$, either (a) holds, in which case $\gamma \subseteq \xi$, or else (b) does, in which case $\gamma \nsubseteq g^{n_{0}} \alpha$. Both cases imply that $\kappa \nsupseteq \gamma$ and hence that $\operatorname{rist}_{U}(\kappa)$ fixes some $\mathfrak{p} \in \gamma$. Then $\operatorname{rist}_{U}(\kappa)$ fixes $\gamma$ because $\mathcal{C}$ is a $U$-invariant partition and, hence, the distinct $U$-translates of $\gamma$ are disjoint. Therefore, we have $\operatorname{rist}_{U}(\kappa) \leqslant \bigcap_{\gamma \in \mathcal{C}} U_{\gamma} \leqslant V$. In particular $g$ rist $_{U}(\kappa) g^{-1} \leqslant U$ and hence $g$ rist $_{U}(\kappa) g^{-1} \leqslant \operatorname{rist}_{U}(g \kappa)$. Therefore, $g$ rist $_{U}(\kappa) g^{-1} \times \operatorname{rist}_{U}\left(g^{n_{0}} \beta\right) \leqslant$ rist $_{U}(\kappa)$ as claimed. 
Let $R=\bigcap_{n \geqslant 0} g^{n} \operatorname{rist}_{U}(\kappa) g^{-n}$. Note that every point that is not fixed by $R$ is contained in $\bigcap_{n \geqslant 0} g^{n} \kappa$. By construction, the intersection $\bigcap_{n \geqslant 0} g^{n} \kappa$ has an empty interior, so the set of fixed points of $R$ is dense in $\mathfrak{S}(\mathcal{A})$. Since $R$ acts by homeomorphisms on $\mathfrak{S}(\mathcal{A})$, the action of $R$ on $\mathfrak{S}(\mathcal{A})$ must be trivial. Since the action of $G$ on $\mathfrak{S}(\mathcal{A})$ is faithful, we have $R=\{1\}$ as claimed. Finally, since $\left(g^{n} \operatorname{rist}_{U}(\kappa) g^{-n}\right)_{n \geqslant 0}$ is a descending chain of compact subgroups with trivial intersection, we have $\operatorname{rist}_{U}(\kappa) \leqslant \operatorname{con}(g)$.

(ii) By part (i), we have $\operatorname{con}(g) \geqslant \operatorname{rist}_{U}\left(g^{n_{0}} \beta\right)=g^{n_{0}} \operatorname{rist}_{U^{\prime}}(\beta) g^{-n_{0}}$, where $U^{\prime}=$ $g^{-n_{0}} U g^{n_{0}}$. Since $\operatorname{con}(g)$ is normalized by $g$, we infer that $\operatorname{rist}_{U^{\prime}}(\beta) \leqslant \operatorname{con}(g)$. Next observe that the conditions that $g \alpha<\alpha$ and $\beta=\alpha \backslash g \alpha$ are equivalent to $g^{-1} \alpha^{\perp}<\alpha^{\perp}$ and $g^{-1} \beta=\alpha^{\perp} \backslash g^{-1} \alpha^{\perp}$. Therefore, the argument of part (i) may be applied to $g^{-1}$ to conclude that

$$
\operatorname{rist}_{W}(\beta) \leqslant \operatorname{con}(g) \cap \operatorname{con}\left(g^{-1}\right),
$$

where $W=g^{-n_{0}} U g^{n_{0}} \cap g^{n_{0}^{\prime}} U g^{-n_{0}^{\prime}}$ for some suitable $n_{0}^{\prime}>0$. This implies that every identity neighbourhood in $G$ contains all but finitely many $\langle g\rangle$-conjugates of $\operatorname{rist}_{W}(\beta)$.

Let $L_{i}=g^{i}$ rist $_{W}(\beta) g^{-i}$ for each $i \in \mathbf{Z}$ and let $K=\overline{\left\langle\bigcup_{i \in \mathbf{Z}} L_{i}\right\rangle}$. Note that for any distinct $i$ and $j$, the sets $g^{i} \beta$ and $g^{j} \beta$ are disjoint, hence the groups $L_{i}$ and $L_{j}$ commute. Moreover each $L_{i}$ is compact, and every identity neighbourhood of $G$ contains all but finitely many $L_{i}$. It follows that $K$ is compact as well, and that moreover the natural homomorphism $\bigoplus_{i \in \mathbf{Z}} L_{i} \rightarrow K$ extends to a continuous homomorphism $\phi: \prod_{i \in \mathbf{Z}} L_{i} \rightarrow K$. The image of $\phi$ is closed and dense in $K$, hence $\phi$ is surjective. The kernel $N$ of $\phi$ commutes with each of the factors $L_{i}$, and is therefore contained in the centre of $\prod_{i \in \mathbf{Z}} L_{i}$. However, the fact that $G$ has a faithful locally weakly decomposable action ensures that $G$ is locally C-stable (see [21, Theorem 5.18]); hence each of the groups $L_{i}$ must have trivial centre, so $\prod_{i \in \mathbf{Z}} L_{i}$ also has trivial centre. Hence $N=\{1\}$, so $\phi$ is an isomorphism of profinite groups. Since $K$ is compact and $g$ acts on $K$ by shifting the factors of the direct product, it is clear that $g$ does not normalize any proper open subgroup of $K$, so $K \leqslant \operatorname{nub}(g)$. We conclude that $\operatorname{con}(g)$ is not closed by [7, Theorem 3.32].

It remains to show that $\langle K, g\rangle \cong K \rtimes\langle g\rangle$ is a closed subgroup of $G$. Notice that $\langle g\rangle$ is a discrete subgroup of $G$, since otherwise $\overline{\langle g\rangle}$ would be compact, which implies that $g$ normalizes a basis of identity neighbourhoods in $G$ and, hence, that $\operatorname{con}(g)$ is trivial. Let now $H=\overline{\langle K, g\rangle}$ and notice that $K$ is a compact normal subgroup of $H$. The image of $\langle g\rangle$ in the quotient $H / K$ is dense since $\langle K \cup g\rangle$ is dense in $H$. Since $\langle g\rangle$ is discrete and torsion-free, it intersects every compact open subgroup trivially. This implies that $H / K$ is discrete and generated by the image of $\langle g\rangle$. Thus $K$ is open in $H$. Therefore, we have $H=\langle K, g\rangle$ and $H$ is indeed closed. 
The fact, seen in the proof, that $\operatorname{con}(g) \geqslant \operatorname{rist}_{W}(\beta)$ for some compact open subgroup $W$ implies the following.

COROLLARY 6.15. Let $G$ be a t.d.l.c. group acting faithfully on a Boolean algebra $\mathcal{A}$, such that the action is locally weakly decomposable. Suppose that there is some $\alpha \in \mathcal{A}$ and $g \in G$ such that $g \alpha<\alpha$; let $\beta=\alpha \backslash g \alpha$. Then the Tits core $G^{\dagger}$ contains $\operatorname{rist}_{U}(\beta)$ for some compact open subgroup $U$ of $G$.

The existence of a minorizing locally weakly decomposable action of $G$ imposes some restrictions on the algebraic structure of $G$.

Proposition 6.16. Let $G$ be a t.d.l.c. group acting faithfully on a Boolean algebra $\mathcal{A}$, such that the action is locally weakly decomposable, and let $\Gamma$ be a subgroup of $G$. Suppose that the action of $\Gamma$ on $\mathcal{A}$ is minorizing of degree $d$. Then $\Gamma$ contains a free submonoid on 2 generators that is discrete in $G$.

Proof. Fix a minorizing set $\mathcal{C}=\left\{\alpha_{1}, \ldots, \alpha_{d}\right\}$ under the action of $\Gamma$ of smallest possible size. Let $\alpha=\alpha_{1}$. Then there exists $g \in \Gamma$ such that $g \alpha<\alpha$ by Lemma 6.13. Moreover, there is $h \in \Gamma$ such that $h \alpha<\alpha \backslash g \alpha$. Let $S$ be the submonoid of $G$ generated by $g$ and $h$; we claim that $S$ is freely generated by $g$ and $h$ and that $\left|S \cap x G_{\alpha}\right| \leqslant 1$ for all $x \in G$. If it happened that either $S$ were not freely generated or $\left|S \cap x G_{\alpha}\right|>1$, there would be elements $x$ and $y$ of $S$ such that $x \alpha=y \alpha$, where $x$ and $y$ are represented as distinct strings $s$ and $t$ respectively in the alphabet $\{g, h\}$. By deleting matching prefixes, we can ensure that the leftmost letters of $s$ and $t$ are different, say $s=g s^{\prime}$ and $t=h t^{\prime}$. But then $x \alpha \leqslant g \alpha$ and $y \alpha \leqslant h \alpha$, so $x \alpha \wedge y \alpha \leqslant g \alpha \wedge h \alpha=0$, contradicting that $x \alpha=y \alpha$. Thus $S$ is free on 2 generators, and it is discrete in $G$ because $\left\{x G_{\alpha} \mid x \in G\right\}$ is an open partition of $G$.

6.5. Minorizing weakly decomposable actions. The aim of this section will be to prove Theorem 6.19 , which gives a sufficient condition for a compactly generated t.d.l.c. group to have a minorizing action on its centralizer lattice. This condition is in particular satisfied by all groups in $\mathscr{S}$ with nontrivial centralizer lattice.

We start by assembling some subsidiary lemmas which will be needed for the proof.

LEMMA 6.17. Let $G$ be a group acting on a set $\Omega$ and let $\Phi \subseteq \Psi$ be subsets of $\Omega$. Let $X$ be a symmetric subset of $G$ such that $G=\left\langle X, \operatorname{Stab}_{G}(\Psi)\right\rangle$. Suppose that

$$
\bigcup\{y \Phi \mid y \in G, y \Phi \subseteq \Psi\} \subseteq \bigcap_{x \in X} x^{-1} \Psi
$$

Then $y \Phi \subseteq \Psi$ for all $y \in G$. 
Proof. Let $l$ be the word length function on $G$ with respect to $X \cup \operatorname{Stab}_{G}(\Psi)$. Suppose there is some $y \in G$ such that $y \Phi \nsubseteq \Psi$; assume $l(y)$ is minimal. Then $y=x z$ where $l(x)=1$ and $l(z)<l(y)$. The minimality of $l(y)$ ensures $x \notin$ $\operatorname{Stab}_{G}(\Psi)$, so $x \in X$. Moreover $z \Phi \subseteq \Psi$, so $z \Phi \subseteq x^{-1} \Psi$ by hypothesis; but then $y \Phi \subseteq \Psi$, a contradiction.

LEMMA 6.18. Let $G$ be a t.d.l.c. group acting faithfully on a Boolean algebra $\mathcal{A}$ such that the action is locally weakly decomposable and let $\Gamma$ be a subgroup of $G$. Suppose the action of $\Gamma$ has a minorizing set $\mathcal{C}$ of size $n$ and no minorizing sets of smaller size. Then there is a subset $\mathcal{D}$ of $\mathcal{A} \backslash\{0\}$ of size $n$, such that for each $\delta \in \mathcal{D}$ there is $\gamma \in \mathcal{C}$ with $\delta \leqslant \gamma$, and satisfying moreover the following property: For all compact open subgroups $U, V$ of $G$ and $\gamma \in \mathcal{A} \backslash\{0\}$, there exist $y \in \Gamma$ and $\delta \in \mathcal{D}$ such that $y \mathrm{rist}_{U}(\delta) y^{-1}$ is a subgroup of $\operatorname{rist}_{V}(\gamma)$ of infinite index.

Proof. By Lemma 6.13, for each $\alpha \in \mathcal{C}$ there is $g_{\alpha} \in \Gamma$ such that $g_{\alpha} \alpha<\alpha$. For each $\alpha \in \mathcal{C}$ choose $n_{0}$ as in Proposition 6.14 and set

$$
\delta_{\alpha}=g_{\alpha}^{n_{0}}\left(\alpha \backslash g_{\alpha} \alpha\right) \text { and } \mathcal{D}=\left\{\delta_{\alpha} \mid \alpha \in \mathcal{C}\right\} .
$$

By construction $|\mathcal{D}| \leqslant n$ and $\mathcal{D}$ is minorizing under the action of $\Gamma$ on $\mathcal{A}$. We infer that $\mathcal{D}$ cannot have fewer than $n$ elements, and so $|\mathcal{D}|=n$.

Fix $\gamma \in \mathcal{A} \backslash\{0\}$. We must show that, for all compact open subgroups $U$ and $V$ of $G$, there are $y \in \Gamma$ and $\delta \in \mathcal{D}$ such that $y \operatorname{rist}_{U}(\delta) y^{-1}<\operatorname{rist}_{V}(\gamma)$. To begin, fix $x \in \Gamma$ and $\alpha \in \mathcal{C}$ such that $x \alpha<\gamma$, and let $K:=x$ rist $_{U}(\alpha) x^{-1} \cap V$, so that $K$ is an open subgroup of $x$ rist $_{U}(\alpha) x^{-1}$. Then, since $x$ rist $_{U}(\alpha) x^{-1}=\operatorname{rist}_{x U x^{-1}}(x \alpha)<$ rist $_{x U x^{-1}}(\gamma)$, we see that $K \leqslant \operatorname{rist}_{V}(\gamma)$.

By Proposition 6.14 there is a closed subset $\kappa$ of $\alpha$ such that $\delta_{\alpha} \subseteq \kappa$ and such that $\left\{x g_{\alpha}^{n} \text { rist }_{U}(\kappa) g_{\alpha}^{-n} x^{-1}\right\}_{n \geqslant 0}$ is a descending chain of closed subgroups of $x \operatorname{rist}_{U}(\alpha) x^{-1}$ with trivial intersection. Hence, $x g_{\alpha}^{n} \operatorname{rist}_{U}\left(\delta_{\alpha}\right) g_{\alpha}^{-n} x^{-1} \leqslant K$ for some $n$ by a standard compactness argument. Setting $y:=x g_{\alpha}^{n}$, it follows that $y \operatorname{rist}_{U}\left(\delta_{\alpha}\right) y^{-1} \leqslant \operatorname{rist}_{V}(\gamma)$. The index $\left[\operatorname{rist}_{V}(\gamma): y \operatorname{rist}_{U}\left(\delta_{\alpha}\right) y^{-1}\right]$ is infinite because $y \delta_{\alpha}=x g_{\alpha}^{n} \delta_{\alpha} \leqslant x \alpha<\gamma$. The claimed minorizing property of $\left\{\right.$ rist $\left._{U}(\delta) \mid \delta \in \mathcal{D}\right\}$ is thus verified.

THEOREM 6.19. Let $G$ be a nontrivial locally $C$-stable compactly generated t.d.l.c. group and let $\mathcal{A} \subseteq \mathcal{L C}(G)$ be a $G$-invariant subalgebra. Suppose that $G$ acts faithfully on $\mathcal{A}$ and that some open subgroup of $G$ has trivial core. Then the set of minimal closed normal subgroups of $G$ is finite; we denote it by $\mathcal{M}=$ $\left\{M_{1}, \ldots, M_{d}\right\}$. Moreover, the following assertions hold, where for each $1 \leqslant i \leqslant d$, the symbol $v_{i}$ denotes the complement of the fixed-point set of $M_{i}$ on $\mathfrak{S}(\mathcal{A})$.

(i) The G-action on $\mathcal{A}$ is minorizing of degree $d$. Indeed, there is a set of $d$ elements in $\mathcal{A}$ that is minorizing for the $G$-action on $\mathcal{L C}(G)$. Moreover, 
the set $\left\{v_{1}, \ldots, v_{d}\right\}$ coincides with the set $\mathcal{U}$ of all minimal nonempty $G$ invariant open subsets of $\mathfrak{S}(\mathcal{A})$, as in Lemma 6.11. In particular, the sets $v_{i}$ are pairwise disjoint.

(ii) Suppose that $\mathcal{A}$ contains the fixed points of the action of $G$ on $\mathcal{L C}(G)$. Let $\alpha_{i}=\left[M_{i}\right]^{\perp^{2}} \in \mathcal{L C}(G)$ for $1 \leqslant i \leqslant d$. Then $\alpha_{i}=\overline{v_{i}}, \alpha_{i} \in \mathcal{A}$ and $\left\{\alpha_{1}, \ldots, \alpha_{d}\right\}$ generates the subalgebra of fixed points of $G$ on $\mathcal{L C}(G)$.

(iii) For each $i \in\{1, \ldots, d\}$ there is an infinite compact subgroup $L_{i}$ of $M_{i}$ which is locally normal in $G$ and such that: for every nontrivial closed locally normal subgroup $K$ of $G$, there are $i \in\{1, \ldots, d\}$ and $g \in G$ such that $g L_{i} g^{-1}$ is a subgroup of $K$ with infinite index.

Proof. Let us first note that any discrete normal subgroup of $G$ would act trivially on $\mathcal{A}$, so in fact $G$ has no nontrivial discrete normal subgroups. The locally Cstable condition then ensures that $G$ has no nontrivial abelian normal subgroups by Proposition 2.7. The hypotheses ensure that there is a compact open subgroup $U$ of $G$ such that $\bigcap_{g \in G} g U g^{-1}=\{1\}$. We can thus apply Proposition 2.12, so that $G$ indeed has only finitely many minimal closed normal subgroups, such that every nontrivial closed normal subgroup contains a minimal one.

(i) We first aim to show that the action of $G$ on $\mathcal{A}$ is minorizing.

Since $G$ is compactly generated, we have $G=\langle U, X\rangle$ for a finite set $X$; we may assume $X=X^{-1}$. Let $V=\bigcap_{x \in X} x^{-1} U x$. By Lemma 6.7, there is a $U$-invariant partition $\mathcal{C}$ of $\mathcal{A}$ such that $\bigcap_{\gamma \in \mathcal{C}} U_{\gamma} \leqslant V$.

Let $\beta \in \mathcal{L C}(G) \backslash\{0\}$. Since $U$ has trivial core in $G$ and $\operatorname{rist}_{U}(\beta)$ is nontrivial, there must exist $g \in G$ such that $\operatorname{rist}_{U}(\beta)$ is not contained in $g^{-1} U g$, or equivalently, $U$ does not contain every $G$-conjugate of $\operatorname{rist}_{U}(\beta)$. The contrapositive of Lemma 6.17 with $\Psi=U$ and $\Phi=\operatorname{rist}_{U}(\beta)$ then ensures the existence of $g \in G$ such that $g \operatorname{rist}_{U}(\beta) g^{-1}$ is contained in $U$ but not $V$. For this $g$, there is some $\gamma \in \mathcal{C}$ such that $g$ rist $_{U}(\beta) g^{-1}$, and hence $\operatorname{rist}_{U}(g \beta)$, does not stabilize $\gamma$. Since rist $_{U}(g \beta)$ fixes $(g \beta)^{\perp}$ pointwise and the partition $\mathcal{C}$ of $\mathcal{A}$ is $U$-invariant, we must have $\gamma \cap(g \beta)^{\perp}=\varnothing=h \gamma \cap(g \beta)^{\perp}$ where $h \in \operatorname{rist}_{U}(g \beta)$ and $h \gamma=\gamma^{\prime}$ for some $\gamma^{\prime} \in \mathcal{C}$ different from $\gamma$. Hence $\gamma<g \beta$ and, since $\beta$ was arbitrary, we have shown that $\bigcup_{\gamma \in \mathcal{C}} G \gamma$ is a minorizing subset of $\mathcal{L C}(G)$.

Since $G$ is minorizing of some finite degree, we can now appeal to Lemma 6.11. Specifically, to show that the action is minorizing of degree $d$, it suffices to prove that $\left\{v_{1}, \ldots, v_{d}\right\}$ is the set of minimal open invariant subsets of $\mathfrak{S}(\mathcal{A})$ as claimed. By definition, the subsets $\left\{v_{1}, \ldots, v_{d}\right\}$ of $\mathfrak{S}(\mathcal{A})$ are open and $G$-invariant. We need to show that they are pairwise disjoint, and that every nonempty open $G$ invariant subset of $\mathfrak{S}(\mathcal{A})$ contains some $v_{i}$. 
Suppose $v_{i} \cap v_{j}$ is nonempty for some distinct $i, j \in\{1, \ldots, d\}$; say $\mathfrak{p} \in v_{i} \cap v_{j}$. There is then $g_{i} \in M_{i}, g_{j} \in M_{j}$ and an open neighbourhood $\delta$ of $\mathfrak{p}$ contained in $\mathfrak{S}(\mathcal{A})$, such that both $g_{i} \delta$ and $g_{j} \delta$ are disjoint from $\delta$. In particular, both $g_{i}$ rist $_{G}(\delta) g_{i}^{-1}$ and $g_{j} \operatorname{rist}_{G}(\delta) g_{j}^{-1}$ have support disjoint from that of $\operatorname{rist}_{G}(\delta)$ and therefore commute with $\operatorname{rist}_{G}(\delta)$ (since the action is faithful). By Lemma 6.8, we conclude that both $M_{i}$ and $M_{j}$ contain the derived group of $\operatorname{rist}_{G}(\delta)$. Since $M_{i} \cap M_{j}=\{1\}$, it follows that $\operatorname{rist}_{G}(\delta)$ is abelian; since $G$ is locally C-stable it then follows by Proposition 2.7 that $\operatorname{rist}_{G}(\delta)$ is trivial. This is impossible, since the action of $G$ on $\mathcal{A}$ is weakly decomposable. Thus the elements of $\left\{v_{1}, \ldots, v_{d}\right\}$ are pairwise disjoint.

On the other hand, if $v$ is a nonempty open $G$-invariant subset of $\mathfrak{S}(\mathcal{A})$, then $\operatorname{rist}_{G}(v)$ is a closed normal subgroup of $G$, which is nontrivial since $\alpha \subseteq v$ for some $\alpha \in \mathcal{A} \backslash\{0\}$. Hence $\operatorname{rist}_{G}(v)$ contains one of the minimal closed normal subgroups, $M_{i}$, and it follows that $v \supseteq v_{i}$.

(ii) For $1 \leqslant i \leqslant d$, set $\alpha_{i}=\left[M_{i}\right]^{\perp^{2}}$. Certainly $\alpha_{i}$ is a fixed point of the action of $G$ on $\mathcal{L C}(G)$, so by hypothesis $\alpha_{i} \in \mathcal{A}$. Since distinct elements of $\mathcal{M}$ commute, we have $\left[M_{i}\right]^{\perp} \geqslant\left[M_{j}\right]$, and so $\left[M_{i}\right] \leqslant\left[M_{i}\right]^{\perp^{2}} \leqslant\left[M_{j}\right]^{\perp}$ for any two distinct elements $M_{i}$ and $M_{j}$ of $\mathcal{M}$. Thus $\left\{\alpha_{1}, \ldots, \alpha_{d}\right\}$ is a pairwise disjoint set of elements of $\mathcal{A}$. By Proposition 6.6, some open subgroup of $M_{i}$ is contained in $\operatorname{rist}_{G}\left(\alpha_{i}\right)$. Since $M_{i}$ is nondiscrete, $\operatorname{rist}_{G}\left(\alpha_{i}\right) \cap M_{i}>\{1\}$. Since rist ${ }_{G}\left(\alpha_{i}\right)$ is closed and normal in $G$ and $M_{i}$ is a nondiscrete minimal nontrivial closed normal subgroup of $G$, we must in fact have $M_{i} \leqslant \operatorname{rist}_{G}\left(\alpha_{i}\right)$. In particular, it follows that $v_{i} \subseteq \alpha_{i}$.

Since the complement of $\bigcup_{i=1}^{d} v_{i}$ is $G$-invariant and does not contain any $v_{i}$, it is nowhere dense in $\mathfrak{S}(\mathcal{A})$ by (i). Therefore, the open set $\alpha_{i} \backslash \overline{v_{i}}$, which is contained in the complement of $\bigcup_{i=1}^{d} v_{i}$, must be empty. This confirms that $v_{i}$ is indeed dense in $\alpha_{i}$ for each $i$. Let $\mathcal{A}^{\prime}$ be the set of fixed points for the action of $G$ on $\mathcal{L C}(G)$. Then $\alpha_{i} \in \mathcal{A}^{\prime}$ for all $i$ by construction and $\mathcal{A}^{\prime} \subseteq \mathcal{A}$ by hypothesis. Given $\beta \in \mathcal{A}^{\prime} \backslash\{0\}$, it follows from part (i) that $v_{i} \subseteq \beta$ for some $i \in\{1, \ldots, d\}$. Since $\beta$ is closed and $v_{i}$ is dense in $\alpha_{i}$, in fact $\beta \geqslant \alpha_{i}$. Since $\mathcal{A}^{\prime}$ is a subalgebra of $\mathcal{A}$, we conclude that $\mathcal{A}^{\prime}$ is the subalgebra generated by $\left\{\alpha_{1}, \ldots, \alpha_{d}\right\}$.

(iii) Let $\mathcal{D}=\left\{\delta_{1}, \ldots, \delta_{d}\right\}$ be as in Lemma 6.18 (with $\Gamma=G$ ). Since $\mathcal{D}$ is a minorizing set for the $G$-action, we must have $\delta_{i} \subseteq v_{i}$ for all $i$ (up to renumbering). For each $i \in\{1, \ldots, d\}$, set $L_{i}=\overline{\left[\operatorname{rist}_{U}\left(\delta_{i}\right) \text {, } \operatorname{rist}_{U}\left(\delta_{i}\right)\right]}$. Note that $L_{i}$ is infinite by virtue of the fact that $G$ is locally C-stable. The quasicentre of $L_{i}$ is a discrete, hence finite, locally normal subgroup of $G$. It must thus be trivial by Proposition 2.7.

Let $K$ be a nontrivial closed locally normal subgroup of $G$. Then there is a compact open subgroup $V$ of $G$ containing $K$ as a closed normal subgroup. Since the $G$-action on $\mathcal{A}$ is faithful, there is a point $\mathfrak{p} \in \mathfrak{S}(\mathcal{A})$ which is not fixed by $K$. Therefore, there exist $k \in K$ and a sufficiently small clopen neighbourhood 
$\gamma \in \mathcal{A}$ of $\mathfrak{p}$ such that $\gamma$ and $k \gamma$ are disjoint. In particular the $\operatorname{groups} \operatorname{rist}_{V}(\gamma)$ and $k$ rist $_{V}(\gamma) k^{-1}$ commute. It then follows from Lemma 6.8 that $K$ contains the derived subgroup of $\operatorname{rist}_{V}(\gamma)$. By Lemma 6.18, there is $g \in G$ and $i \in\{1, \ldots, d\}$ such that $g$ rist $_{U}\left(\delta_{i}\right) g^{-1}$ is contained in $\operatorname{rist}_{V}(\gamma)$, whence $K$ contains $g L_{i} g^{-1}$. The construction ensures that the index of $g L_{i} g^{-1}$ in $K$ is infinite because $\mathrm{kgL}_{i} g^{-1} k^{-1}$ is infinite, is contained in $K$ and intersects trivially with $g L_{i} g^{-1}$.

Let $1 \leqslant i \leqslant d$. Since $M_{i}$ is a nontrivial closed locally normal subgroup of $G$, we have $g L_{j} g<M_{i}$ for some $g \in G$ and $1 \leqslant j \leqslant d$; since $M_{i}$ is normal, $L_{j}<M_{i}$. Given that $\delta_{j} \subseteq v_{j}$ and $L_{j}$ fixes every point outside of $\delta_{j}$, we see that $L_{j} \cap M_{i}=\{1\}$ unless $i=j$. Thus we must have $L_{i}<M_{i}$.

6.6. Properties of simple t.d.l.c. groups. We now focus on consequences of Theorem 6.19 for the structure of groups in $\mathscr{S}$. For clarity we highlight the special case of Theorem 6.19 when $G \in \mathscr{S}$.

Corollary 6.20. Let $G \in \mathscr{S}$ and let $\mathcal{A}=\mathcal{L C}(G)$ or $\mathcal{A}=\mathcal{L} \mathcal{D}(G)$. Assume that $|\mathcal{A}|>2$. Then $G$ has a minorizing orbit on $\mathcal{A}$. Moreover, there is an infinite compact locally normal subgroup $L$ of $G$ with the following property: for every nontrivial closed locally normal subgroup $K$ of $G$, there exists $g \in G$ such that $\mathrm{gLg}^{-1}$ is a subgroup of $K$ of infinite index.

Proof. In view of Corollary 5.12, the $G$-action on $\mathcal{A}$ is faithful. Since $G$ is topologically simple, we are in the situation of Theorem 6.19 where the number of minimal closed normal subgroups is 1 . The conclusions are now immediate from Theorem 6.19 .

REMARK 6.21. For the conclusion of Corollary 6.20, in general $L$ cannot be chosen to be a rigid stabilizer of the action of $G$ on $\mathcal{L C}(G)$, as demonstrated by the following example. Let $T$ be the regular tree of degree $d \geqslant 6$, let $G$ be the simple group $\operatorname{Aut}(T)^{+}$generated by edge stabilizers in $G$. Let $e \in E T$ and let $L$ be the fixator of the set of vertices closer to $t(e)$ than $o(e)$. Then certainly $G \in \mathscr{S}$, $\mathcal{L C}(G)$ is nontrivial, and both $L$ and $K:=\overline{[L, L]}$ are infinite nonopen compact locally normal subgroups of $G$. Indeed, considered as an element of $\mathcal{L C}(G)$, then $[L]$ is minorizing under the action of $G$. However, for all $v \in V T$, the permutation induced by $K_{v}$ on the neighbours of $v$ is even, whereas elements of $L$ can induce odd permutations on the neighbours of infinitely many vertices. Thus $L$ is not conjugate in $G$ to a subgroup of $K$; indeed, there does not exist $g \in G$ such that $\left[g L g^{-1}\right] \leqslant[K]$. Since $[L]$ is minorizing in $\mathcal{L C}(G)$, the same argument shows that $g \mathrm{Mg}^{-1} \not \leq K$ for all $g \in G$, where $M$ is any compact representative of any given nonzero element of $\mathcal{L C}(G)$. 
We can prove a stronger version of Theorem B(iv) given $G \in \mathscr{S}$ with property (S2), if we make the additional assumption that $\mathcal{L C}(G)$ is nontrivial. In this case we obtain two global invariants of the group $G$ that control the 'size' of locally normal subgroups up to conjugation.

Corollary 6.22. Let $G \in \mathscr{S}$ be such that $|\mathcal{L C}(G)|>2$. Suppose that every infinite commensurated compact subgroup of $G$ is open (this is automatic if $G$ is abstractly simple, see Theorem 3.9). Then there are natural numbers $m$ and $n$ and an open subgroup $U$ of $G$ such that the following holds, for every nontrivial closed locally normal subgroup $K$ of $G$ :

(i) There is a set $\left\{g_{1}, \ldots, g_{m}\right\}$ of m elements of $G$ such that the (not necessarily direct) product $\prod_{i=1}^{m} g_{i} K g_{i}^{-1}$ contains $U$;

(ii) There is a set $\left\{h_{1}, \ldots, h_{n}\right\}$ of n elements of $G$ such that

$$
G=\left\langle h_{i} K h_{i}^{-1} \mid 1 \leqslant i \leqslant n\right\rangle .
$$

Proof. Let $L$ be the compact locally normal subgroup of $G$ given by Corollary 6.20.

By Theorem 5.2, there is a finite set $\left\{g_{1}, \ldots, g_{m}\right\}$ of $G$ such that the product $\prod_{i=1}^{m} g_{i} L g_{i}^{-1}$ contains a nontrivial commensurated compact locally normal subgroup $U$ of $G$. Then $U$ is open by hypothesis. Since $G$ is compactly generated and topologically simple and $U$ is open, $G$ is generated by finitely many conjugates of $U$; since $U$ is contained in a subgroup generated by finitely many conjugates of $L$, finitely many conjugates of $L$ will also suffice to generate $G$. In other words,

$$
G=\left\langle h_{i} L h_{i}^{-1} \mid 1 \leqslant i \leqslant n\right\rangle
$$

for some finite set $\left\{h_{1}, \ldots, h_{n}\right\}$.

We have now proved (i) and (ii) for a specific compact locally normal subgroup $L$. But in fact the choice of $L$ simultaneously provides a solution for all nontrivial compact locally normal subgroups $K$ : given such a $K$ we have $r^{-1} L r \leqslant K$ for some $r \in G$, in other words, $L \leqslant r K r^{-1}$, and therefore $\prod_{i=1}^{m} g_{i} r K r^{-1} g_{i}^{-1}$ contains $U$ and $\left\{h_{i} r K r^{-1} h_{i}^{-1} \mid 1 \leqslant i \leqslant n\right\}$ generates $G$. Thus the group $U$ and the numbers $m$ and $n$ can be chosen independently of the choice of $K$.

We can now also prove Theorems G and N.

Proof of Theorem $G$. Let $G \in \mathscr{S}$ and let $\alpha \in \mathcal{L} \mathcal{N}(G)$. We suppose that $\alpha$ is a minimal nonzero element of $\mathcal{L N}(G)$, in other words $\alpha$ is a minimal element of $\mathcal{F}:=\mathcal{L} \mathcal{N}(G) \backslash\{0\}$; this will clearly be the case if $\alpha$ has a h.j.i. representative. 
Suppose $\mathcal{L C}(G)$ is nontrivial. Then by Corollary 6.20, every infinite compact locally normal subgroup $K$ of $G$ contains an infinite closed locally normal subgroup $L$ of $G$, such that $L$ has infinite index in $K$. In particular, there cannot be any minimal nonzero element of $\mathcal{L N}(G)$, contradicting the existence of $\alpha$. Thus $\mathcal{L C}(G)$ is trivial and $\mathcal{F}$ is a filter by Lemma 5.10. Since $\alpha$ is minimal in $\mathcal{F}$, we see that in fact $\mathcal{F}$ is a principal filter, with $\alpha$ the unique least element of $\mathcal{F}$. If $\alpha=\infty$ then $\mathcal{L N}(G)=\{0, \infty\}$, so $G$ is locally h.j.i.; otherwise, we see from Theorem $\mathrm{F}$ that $G$ is of atomic type. Thus (ii) is proved. Theorem $\mathrm{F}$ also ensures that $G$ acts trivially on $\mathcal{L N}(G)$; part (i) follows immediately.

From now on we may suppose that $G$ is of atomic type. It remains to show that there exists a continuous homomorphism $\phi: S \rightarrow G$ such that $S \in \mathscr{S}, S$ is not of atomic type and $\phi(S)$ is a proper dense normal subgroup of $G$. Consider a compact representative $K$ of $\alpha$. Since $\alpha<\infty$, we see that $K$ is not open in $G$. However, $\alpha$ is fixed by $G$, so $K$ is commensurated in $G$. Proposition 5.20 then provides a continuous homomorphism $\phi: S \rightarrow G$ such that $S \in \mathscr{S}$ and $\phi(S)$ is normal in $G$. Moreover, $\phi(S)=\left\langle\langle M\rangle_{G}\right.$ for some infinite compact locally normal subgroup $M$ of $G$. We see from the construction of $S$ that $0<[M] \leqslant \alpha$, so by the minimality of $\alpha$, in fact $M$ is a representative of $\alpha$. We see that $M$ has countable index in $\phi(S)$, but uncountable index in $G$, so $\phi(S)<G$. In particular, $G$ is not abstractly simple.

Suppose $\mathcal{L} \mathcal{N}(S)$ has an atom $\beta$. Then the same argument as for $G$ shows that $\beta$ is unique, and thus the commensurability class of $\phi(K)$ is preserved by conjugation in $G$, where $K$ is any compact representative of $\beta$. By Lemma 3.10 we can find a compact locally normal representative $L$ of $\beta$ such that $\phi(L)$ is locally normal in $G$. Minimality of $\alpha$ then ensures $[\phi(L)]=\alpha$, which in turn implies that $L$ is open in $S$, and hence $\beta$ is the greatest element of $\mathcal{L N}(S)$. In this case $\mathcal{L N}(S)=\{0, \infty\}$, so $S$ is locally h.j.i.; in particular, $S$ is not of atomic type.

Proof of Theorem $N$ and Corollary $O$. If there is a subgroup $R=\prod_{i \in \mathbf{Z}} K_{i} \rtimes\langle g\rangle$ of the form described, it is clear that $\mathcal{L C}(G)$ is nontrivial, since for instance the centralizer of the factor $K_{0}$ is neither open nor discrete.

Conversely, suppose $\mathcal{L C}(G) \neq\{0, \infty\}$. By Corollary 6.20, $G$ has a minorizing orbit on $\mathcal{L C}(G)$; in particular, there exists $\alpha \in \mathcal{L C}(G)$ and $g \in G$ such that $g \alpha<\alpha$. Hence by Proposition 6.14, there is a closed subgroup $R$ of $G$ of the required form. Proposition 6.14 also ensures that $\operatorname{con}(g)$ is not closed.

Proof of Corollary $R$. Consider the subgroup $R=\prod_{i \in \mathbf{Z}} K_{i} \rtimes\langle g\rangle$ afforded by Theorem N. Since $K_{0}$ is an infinite locally normal subgroup of $G$, it is nonabelian by Theorem A. Choose any two elements $a, b \in K_{0}$ which do not commute. Then the group $\Gamma=\langle g, a, b\rangle$ is isomorphic to the wreath product $\langle a, b\rangle_{2} \mathbf{Z}$. Since $\langle a$, 
$b\rangle$ is not abelian, that wreath product is not redisually finite by [30]. A wellknown theorem of Mal'cev [38] ensures that finitely generated linear groups are residually finite. The result follows.

6.7. Minimality and strong proximality. Recall that the action of a group $G$ on a compact space $\Omega$ is minimal if every orbit is dense. It is called proximal if every pair $\{\eta, \xi\} \subset \Omega$ is compressible to some $\omega \in \Omega$ (see Section 6.3 for the definition of compressibility), and strongly proximal if for any probability measure $\mu$ on $\Omega$, the $G$-orbit $G \mu$ contains Dirac measures in its closure in the space of probability measures on $\Omega$. Our aim in this section is to establish minimality and strong proximality for the action of $G$ on $\mathfrak{S}(\mathcal{L C}(G))$ in the case that $G \in \mathscr{S}$, which will lead to proofs of Theorem J and its corollaries.

Let us first consider dense orbits in $\mathfrak{S}(\mathcal{L C}(G))$.

THEOREM 6.23. Let $G$ be a topologically simple, locally $C$-stable t.d.l.c.s.c. group. Suppose $\mathcal{A}$ is a $G$-invariant subalgebra of $\mathcal{L C}(G)$ for which $|\mathcal{A}|>2$. Then:

(i) The action of $G$ on $\mathfrak{S}(\mathcal{A})$ has a dense orbit; indeed, every orbit $\omega$ of $G$ on $\mathfrak{S}(\mathcal{A})$ is either a singleton or dense in $\mathfrak{S}(\mathcal{A})$.

(ii) If $G$ is compactly generated, then the action of $G$ on $\mathfrak{S}(\mathcal{A})$ is minimal.

(iii) Suppose that $\mathcal{A}$ is countable. If $G$ is abstractly simple, then the action of $G$ on $\mathfrak{S}(\mathcal{A})$ is minimal.

Proof. Since $G$ is locally C-stable, $\mathrm{QZ}(G)$ is discrete. The existence of a nontrivial subalgebra of $\mathcal{L C}(G)$ ensures that $G$ is not discrete; since $G$ is topologically simple, it follows that $\mathrm{QZ}(G)=\{1\}$. By Corollary 5.12, the action of $G$ on $\mathcal{A}$ is faithful, with $\mathcal{A}^{G}=\{0, \infty\}$. The action is locally weakly decomposable by Proposition 6.6.

(i) The action of $G$ on $\mathfrak{S}(\mathcal{A})$ is faithful because the action on $\mathcal{A}$ is faithful. Let $\omega$ be an orbit of $G$ such that $|\omega|>1$; such an orbit exists since the action of $G$ on $\mathfrak{S}(\mathcal{A})$ is nontrivial. Since $G$ is topologically simple, $G$ acts faithfully on $\omega$ and hence $\operatorname{rist}_{G}(\mathfrak{S}(\mathcal{A}) \backslash \omega)=\{1\}$. Since the $G$-action is weakly decomposable, we conclude that $\omega$ is dense.

For the proof of parts (ii) and (iii), let $\kappa$ be the set of fixed points of $G$ acting on $\mathfrak{S}(\mathcal{A})$. The fact that $G$ acts nontrivially on $\mathfrak{S}(\mathcal{A})$ ensures that $\kappa$ is not dense.

(ii) Let $\beta \in \mathcal{A}$ be such that $\beta>0$ and $\beta \cap \kappa=\varnothing$; such a $\beta$ exists because $\kappa$ is not dense. Considering $\beta$ as an element of $\mathcal{L N}(G)$, by Theorem 5.2 there exists a finite subset $\left\{g_{1}, \ldots, g_{n}\right\}$ of $G$ such that $\gamma=\bigvee_{i=1}^{n} g_{i} \beta$ is fixed by $G$. Thus $\gamma^{\perp}$ is 
a fixed point of the action of $G$ on $\mathcal{L C}(G)$; since clearly $\gamma>0$, we have $\gamma^{\perp}<\infty$ and hence $\gamma^{\perp}=0$. We now observe that the set $\left\{g_{1} \beta, \ldots, g_{n} \beta\right\}$ has a least upper bound $\delta$ in $\mathcal{A}$, since $\mathcal{A}$ is a lattice. Since $\mathcal{A}$ is a subset of $\mathcal{L N}(G)$ we have $\gamma \leqslant \delta$, so $\delta^{\perp} \leqslant \gamma^{\perp}=0$. Thus $\delta=\infty$. At the same time, since $\kappa$ is $G$-invariant, we have $g_{i} \beta \cap \kappa=\varnothing$ for all $i$, so $\delta \cap \kappa=\varnothing$, and hence $\kappa=\varnothing$. We conclude by part (i) that every orbit of $G$ on $\mathfrak{S}(\mathcal{A})$ is dense, in other words the action is minimal.

(iii) We prove this part in contrapositive form. Suppose that the action of $G$ on $\mathfrak{S}(\mathcal{A})$ is not minimal, that is, not all $G$-orbits are dense. By part (i), $\kappa$ is nonempty. Using this fact, we can exhibit a proper nontrivial normal subgroup of $G$ to show that $G$ is not abstractly simple. Define

$$
N=\bigcup_{\alpha \in \mathcal{A}, \alpha \cap \kappa=\varnothing} \operatorname{rist}_{G}(\alpha),
$$

a directed union of subgroups of $G$ that is normal because $\kappa$ is $G$-invariant and nontrivial because the $G$-action is weakly decomposable. Thus $N$ is a dense normal subgroup of $G$; it remains to show that $N \neq G$.

Let $U$ be a compact open subgroup of $G$ and suppose $N \geqslant U$. Since $\mathcal{A}$ is countable, (6.1) is a countable directed union of closed sets. The Baire Category Theorem then implies that there is $\alpha \in \mathcal{A}$ intersecting $\kappa$ trivially and such that rist $_{U}(\alpha)$ is open in $U$.

Since $\alpha \cap \kappa=\varnothing$ we have $\alpha \neq \infty$ and so $\alpha^{\perp}>0$. In other words, taking $K$ to be a compact representative of $\alpha$, then $\mathrm{C}_{G}(K)$ is nondiscrete since $\left[\mathrm{C}_{G}(K)\right]=$ $\alpha^{\perp}$ by Proposition 6.6. Then faithfulness of the action of $G$ on $\mathcal{A}$ implies that

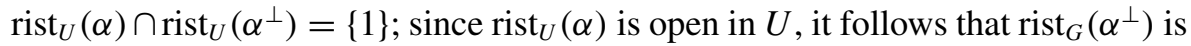
discrete. This is absurd as we clearly have $\mathrm{C}_{G}(K) \leqslant \operatorname{rist}_{G}\left(\alpha^{\perp}\right)$. We conclude that $N \ngtr U$ and hence that $N \neq G$ as claimed.

We now give a sufficient condition for an action to be strongly proximal.

PROPOSITION 6.24. Let $G$ be a topologically simple locally compact group acting continuously by homeomorphisms on a profinite space $\Omega$. Assume that the action is minimal and locally weakly decomposable, and that there is a nonempty compressible open subset. Then the G-action is strongly proximal.

Proof. Every nonempty open subset contains a nonempty clopen subset. Moreover, by minimality every $G$-orbit visits every nonempty clopen subset. Therefore, our hypotheses imply that every point of $\Omega$ admits a compressible clopen neighbourhood.

By [12, Theorem 4.5] (see also [39, Proposition VI.1.6] for the special case when $\Omega$ is metrizable), an action of a group on a compact space is strongly 
proximal as soon as that action is minimal, proximal and contains a compressible open set. Therefore, in order to prove the proposition, it suffices to show that every pair $\{\eta, \xi\}$ in $\Omega$ is compressible. Given any pair $\{\eta, \xi\}$, and let $\alpha$ be a compressible clopen neighbourhood of $\eta$.

Let $N$ be the subgroup of $G$ generated by $\bigcup_{g \in G} \operatorname{rist}_{G}(g \alpha)$. Then $N$ is a nontrivial normal subgroup of $G$, which is thus dense. Since every $G$-orbit on $\Omega$ is dense, so is every $N$-orbit. In particular there is some $g \in N$ such that $g \xi \in \alpha$. Among all the $g \in N$ with $g \xi \in \alpha$, we choose one of minimal word length with respect to the generating set $\bigcup_{g \in G} \operatorname{rist}_{G}(g \alpha)$. Write $g=r_{n} \ldots r_{1}$ as a product of elements $r_{i} \in \operatorname{rist}_{G}\left(\alpha_{i}\right)$, where $\alpha_{i}$ belongs to the $G$-orbit of $\alpha$. Set $g_{j}=r_{j} \ldots r_{1}$ for all $j=\{1, \ldots, n\}$ and set $g_{0}=1$. By the minimality of $n$, we have $g_{j-1} \xi \in \alpha_{j}$ for all $j>0$, since otherwise we would have $g_{j} \xi=g_{j-1} \xi$ and hence

$$
r_{n} \ldots r_{j+1} g_{j-1} \xi=g \xi \in \alpha,
$$

which contradicts the minimality of $n$.

Now we distinguish two cases. Assume first that $\eta \notin \alpha_{j}$ for all $j \in\{1, \ldots, n\}$. It then follows that $r_{j}$ fixes $\eta$ for all $j$, and hence so does $g$. Therefore, we have $g(\{\eta, \xi\})=\{\eta, g . \xi\} \subset \alpha$. Since $\alpha$ is compressible, this implies that the pair $\{\eta, \xi\}$ is also compressible, and we are done.

Assume next that there is some $j>0$ such that $\eta \in \alpha_{j}$; in this case, let $i=$ $\min \left\{j>0 \mid \eta \in \alpha_{j}\right\}$. Thus $r_{j}$ fixes $\eta$ for all $j<i$, and hence $g_{i-1} \eta=\eta$. In particular $g_{i-1} \eta \in \alpha_{i}$. Moreover, we have seen above that $g_{i-1} \xi \in \alpha_{i}$. Therefore, $g_{i-1}(\{\eta, \xi\}) \subset \alpha_{i}$, and hence $\{\eta, \xi\}$ is compressible because $\alpha_{i}$ is so.

Thus every pair in $\Omega$ is compressible, and the $G$-action is indeed proximal.

Proof of Theorem J. By Theorem A and Proposition 2.7, the group G is locally Cstable. Therefore, the hypotheses of Theorem 6.23 are fulfilled. The $G$-action on $\Omega=\mathfrak{S}(\mathcal{L C}(G))$ is continuous by Lemma 6.2. It is locally weakly decomposable by Proposition 6.6 (in particular it is smooth), and minimal by Theorem 6.23(ii). Moreover, by Corollary 6.20 there is a minorizing $G$-orbit in $\mathcal{L C}(G)$. This implies that the corresponding clopen subset of $\Omega$ is compressible by Lemma 6.10. The strong proximality follows from Proposition 6.24.

This proves (i). Since $G$ is locally C-stable with trivial quasicentre, the assertion (ii) follows from (i) together with [21, Theorem II(ii)].

Proof of Corollary L. Follows from Theorem J and Proposition 6.16.

Proof of Corollary $M$. If $\mathcal{L C}(G)$ is nontrivial, then the $G$-action on $\mathcal{L C}(G)$ is nontrivial by Theorem J, hence faithful since $G$ is topologically simple. The uniqueness of the topology on $G$ among $\sigma$-compact locally compact topologies 
follows from [21, Corollary 7.6]. Indeed, the same argument shows that the topology of $G$ is the only Polish topology compatible with the group structure.

Recall that fixed points in the structure lattice correspond to conjugationinvariant commensurability classes of compact locally normal subgroups. If in addition $G$ is abstractly simple, then the only fixed points of $G$ in the structure lattice are the trivial ones by Theorem 3.9; the desired statement therefore also follows from [21, Corollary 7.6].

We recall that strong proximality may be viewed as an antipodal condition to amenability. In fact we have the following result due to Furstenberg.

Proposition 6.25. Let $G$ be a locally compact group admitting a closed amenable subgroup A such that $G / A$ is compact. Let also $\Omega$ be a compact $G$ space which is minimal and strongly proximal. Then A fixes a point in $\Omega$, and the $G$-action on $\Omega$ is transitive.

Proof. By [27, Proposition 4.4], for every compact $G$-space $Z$ such that the $G$ action on $Z$ is minimal and strongly proximal, there is a unique $G$-equivariant continuous map $\psi: G / A \rightarrow Z$. Since the $G$-action on $Z$ is minimal, it follows that the continuous map $\psi$ must be surjective. The image of the trivial coset under $\psi$ is a $G$-fixed point in $Z$, and the transitivity of $G$ on $G / A$ implies the transitivity on $Z$.

Corollary K now follows immediately from Theorem J and Proposition 6.25.

REMARK 6.26. Let $G$ be a compactly generated, topologically simple t.d.l.c. group and suppose $|\mathcal{L C}(G)|>2$. It seems plausible that every nontrivial orbit of $G$ on $\mathcal{L C}(G)$ is minorizing. This would imply that, for every closed subset $X \neq$ $\mathfrak{S}(\mathcal{L C}(G))$, there is a net $\left\{g_{i}\right\} \subset G$ such that $\lim _{i} g_{i} X$ is a singleton. By definition, this means that the action of $G$ on $\mathfrak{S}(\mathcal{L C}(G))$ is extremely proximal. That condition implies in particular that $G$ contains nonabelian discrete free subgroups by [29, Theorem 3.4]. In light of the results we have so far, an equivalent formulation of the property 'every nontrivial orbit of $G$ on $\mathcal{L C}(G)$ is minorizing' in the present context is the following:

Let $K$ be a compact locally normal subgroup of $G$ such that $K>1$. Then there is some $g \in G$ such that $\left[\mathrm{C}_{G}(K), \mathrm{C}_{G}\left(g K g^{-1}\right)\right]=\{1\}$.

6.8. Abstract simplicity. Our main aim in this subsection is to complete the proofs of Theorems P and Q. We start by recalling the main result from [20]. 
THEOREM 6.27 [20, Theorem 1.1]. Let $G$ be a t.d.l.c. group and let $D$ be a dense subgroup of $G$ such that $G^{\dagger} \leqslant \mathrm{N}_{G}(D)$. Then $G^{\dagger} \leqslant D$. In particular, every dense subnormal subgroup of $G$ contains the Tits core of $G$.

Proof of Theorem P. Suppose case (i) holds. By Corollary 6.20, the group $G$ has a minorizing orbit on $\mathcal{L C}(G)$. By Corollary 6.15, the Tits core $G^{\dagger}$ contains $K=$ $\operatorname{rist}_{U}(\beta)$ for some compact open subgroup $U<G$ and some nonzero $\beta \in \mathcal{L C}(G)$. Therefore, $G^{\dagger}$ also contains an infinite commensurated compact locally normal subgroup $L$ by Lemma 5.1. Since $G^{\dagger}$ is contained in every dense normal subgroup of $G$ by Theorem 6.27, we infer that every dense normal subgroup contains the infinite commensurated compact locally normal subgroup $L$. Hence property (S1) holds.

Suppose case (ii) holds, that is, there is a topologically finitely generated compact open subgroup $U$ of $G$. We recall that by Proposition 4.6, the composition factors of $U$ are of bounded order. Now, given a dense normal subgroup $N$ of $G$, the intersection $N \cap U$ is a dense normal subgroup of $U$, and must thus contain the closed subgroup $[U, U]$ by Theorem 5.21. Notice that $[U, U]$ is infinite by Theorem $\mathrm{A}$, and thus represents a nonzero element of the structure lattice. By Lemma 5.1, it follows that $N$ contains a representative of a nonzero $G$-fixed point in $\mathcal{L} \mathcal{N}(G)$. Thus $G$ has property (S1).

The conclusion about abstract simplicity follows by Theorem 3.9.

We next obtain a sufficient condition for the Tits core of a t.d.l.c. group to be open, which we can use to prove that a large class of topologically simple groups are in fact abstractly simple via the following consequence of Theorem 6.27.

COROLLARY 6.28. Let $G$ be a nondiscrete topologically simple t.d.l.c. group such that $G^{\dagger}$ is open in $G$. Then $G=G^{\dagger}$ and $G$ is abstractly simple.

Proof. We see that $G^{\dagger}$ is a nontrivial closed normal subgroup of $G$, so $G=G^{\dagger}$ by topological simplicity. Now given any nontrivial normal subgroup $N$ of $G$, then $N$ is dense in $G$ by topological simplicity, so $N \geqslant G^{\dagger}$ by Theorem 6.27 and hence $N=G$. Thus $G$ is abstractly simple.

Proposition 6.29. Let $G$ be a locally $C$-stable t.d.l.c. group with $\mathrm{QZ}(G)=\{1\}$ and let $\mathcal{A}$ be a subalgebra of $\mathcal{L D}(G)$. Suppose that the action of $G$ on $\mathfrak{S}(\mathcal{A})$ is faithful and minimal, and that $g \alpha<\alpha$ for some $g \in G$ and $\alpha \in \mathcal{A}$. Then $G^{\dagger}$ is open in $G$.

Proof. Let $\beta=\alpha \backslash g \alpha$. By Corollary 6.15 we have $\operatorname{rist}_{U}(\beta) \leqslant G^{\dagger}$ for some compact open subgroup $U$ of $G$. The minimality of the $G$-action on $\mathfrak{S}(\mathcal{A})$ implies 
that $\bigcup_{g \in G} g \beta=\mathfrak{S}(\mathcal{A})$. Moreover the compactness of $\mathfrak{S}(\mathcal{A})$ implies that in fact $\mathfrak{S}(\mathcal{A})=\bigcup_{i=1}^{n} g_{i} \beta$ for some finite set $\left\{g_{1}, \ldots, g_{n}\right\}$. Hence $\infty=\bigvee_{i=1}^{n} g_{i} \beta$. Recall that $\gamma=\left[\operatorname{rist}_{V}(\gamma)\right]$ for all compact open subgroups $V$ and all $\gamma \in \mathcal{L D}(G)$ (see Proposition 6.6). Since the join in $\mathcal{L D}(G)$ corresponds to taking products of representatives, we see that

$$
\left\langle g_{i} \operatorname{rist}_{U}(\beta) g_{i}^{-1} \mid 1 \leqslant i \leqslant n\right\rangle
$$

is an open subgroup of $G$. Since $G^{\dagger}$ contains this subgroup, it is open too.

Proof of Theorem $Q$. In all cases, $G$ is locally C-stable by Theorem 5.3.

Suppose case (i) holds, that is, $\mathcal{L D}(G) \neq\{0, \infty\}$. Since $G \in \mathscr{S}$, the action of $G$ on $\mathcal{L D}(G)$ is faithful by Corollary 5.12. By Theorem 6.23(ii), the action of $G$ on $\mathfrak{S}(\mathcal{L D}(G))$ is faithful and minimal. Moreover it has a minorizing orbit by Corollary 6.20. In particular, there exists $g \in G$ and $\alpha \in \mathcal{L D}(G)$ such that $g \alpha<\alpha$.

It has thus been shown that $G$ satisfies the hypotheses of Proposition 6.29. Hence $G^{\dagger}$ is open in $G$, whence $G^{\dagger}=G$ and $G$ is abstractly simple by Corollary 6.28.

Suppose case (ii) holds and let $U$ be a compact open subgroup $U$ of $G$ such that $U$ is finitely generated as a profinite group and $\overline{[U, U]}$ is open in $G$. In fact $[U, U]=\overline{[U, U]}$ by Theorem 5.21 , so $[U, U]$ is open in $G$. We conclude with a similar argument as in the proof of Theorem P: any dense normal subgroup $N$ of $G$ must contain $[U, U]$ by Theorem 5.21, and hence $N$ is open in $G$. Since $N$ is dense, we have $N=G$. Thus $G$ has no proper dense normal subgroups; since $G$ is topologically simple by hypothesis, it follows that $G$ is abstractly simple.

Finally, suppose case (iii) holds, that is, $\mathcal{L N}(G)^{G}=\{0, \infty\}$ and some nontrivial compact locally normal subgroup $K$ of $G$ is topologically finitely generated. It follows that every compact representative of $\alpha=[K]$ is topologically finitely generated, and indeed every representative of $\beta \in \mathcal{L N}(G)$ is topologically finitely generated whenever $\beta$ can be represented as a finite join of $G$-conjugates of $\alpha$. Thus by Theorem 5.2, there must be a nontrivial compact locally normal subgroup $L$ that is topologically finitely generated and commensurated by $G$. Since we assume that $G$ does not have any nontrivial fixed point in the structure lattice, we infer that $L$ is open in $G$. Thus $G$ is abstractly simple by Theorem P(ii).

REMARK 6.30. The local decomposition lattice is a sublattice of the structure lattice. In particular, the least upper bound operation in $\mathcal{L D}(G)$ satisfies

$$
\left[H_{1}\right] \vee\left[H_{2}\right]=\left[H_{1} H_{2}\right] \text {. }
$$


This identity plays a crucial role in Proposition 6.29. By contrast, in $\mathcal{L C}(G)$ we have only that $\left[H_{1}\right] \vee\left[H_{2}\right]=\left[H_{1} H_{2}\right]^{\perp^{2}}$, so for groups $G \in \mathscr{S}$ of weakly decomposable type, the strategy employed in Proposition 6.29 is not sufficient to show that $G^{\dagger}$ is open. Indeed, in this case we do not know if $G^{\dagger}$ is necessarily open (although it is certainly nontrivial).

\section{Acknowledgements}

We are grateful to Simon Smith for communicating to us the details of his construction of uncountably many isomorphism classes in $\mathscr{S}$ from [59] prior to publication. We also thank the anonymous referee for very thorough and helpful reports on the successive preliminary incarnations of this paper, with many good suggestions for improvements. This research was supported by an F.R.S.-FNRS research associate, funded in part by the ERC grant \#278469, and by the ARC Discovery Projects DP0984342 and DP120100996.

\section{Appendix A. Known sources of examples}

Until very recently, the only known results on the class $\mathscr{S}$ focused on specific families of examples studied with the help of the extra structure provided by the very construction of these families. Although the purpose of this paper is to lay the foundations of a study of general groups in $\mathscr{S}$, for reference we list the sources of examples of particular groups in $\mathscr{S}$ that we are aware of at the time of writing.

- Isotropic semialgebraic groups over non-Archimedean local fields. Let $k$ be a non-Archimedean local field, and $\mathbf{G}$ be an algebraic group defined over $k$. Assume that $\mathbf{G}$ is noncommutative, almost $k$-simple and $k$-isotropic. The group $\mathbf{G}(k)$, endowed with the Hausdorff topology induced by $k$, is a compactly generated t.d.l.c. group (see [39, Corollary I.2.3.5]). Let $\mathbf{G}(k)^{+}$be the normal subgroup of $\mathbf{G}(k)$ generated by the unipotent radicals of $k$-defined parabolic subgroups. Then $\mathbf{G}(k)^{+}$is a closed cocompact subgroup of $\mathbf{G}(k)$ by [10, Section 6.14], and the quotient of $\mathbf{G}(k)^{+}$by its centre $Z$ is abstractly simple by the main result of [61]. In particular $\mathbf{G}(k)^{+} / Z$ belongs to the class $\mathscr{S}$. All of these simple algebraic groups have been classified by Tits [63]; each of them acts properly and cocompactly on a locally finite Euclidean building constructed by Bruhat and Tits [13]. We also remark that if $k$ is a $p$-adic field, then the simple group $\mathbf{G}(k)^{+} / Z$ also carries the structure of a $p$-adic Lie group. Conversely, every compactly generated topologically simple $p$-adic Lie group is of that form. (Every topologically simple $p$-adic Lie group whose adjoint representation is nontrivial is isomorphic to a $p$-adic simple algebraic group of 
the form $\mathbf{G}(k)^{+} / Z$, and is thus compactly generated, see [23, Proposition 6.5] (we emphasize that analytic $p$-adic groups are implicitly assumed to be linear in the latter reference). It is however not known whether a topologically simple $p$-adic Lie group can have a trivial adjoint representation (or even be one-dimensional, hence locally isomorphic to $\mathbf{Z}_{p}$ ). A topologically simple $p$ adic Lie group whose adjoint representation is trivial must have an abelian Lie algebra and, hence, be locally abelian. Therefore, it cannot be compactly generated by Theorem A.) More generally, it follows from [22, Corollary 1.4] that simple algebraic groups are the only members of the class $\mathscr{S}$ that are (locally) linear over a local field.

- Complete Kac-Moody groups of indecomposable type over finite fields. These groups are constructed as completions of minimal Kac-Moody groups over finite fields defined by Tits [64], with respect to a suitable topology. The resulting locally compact groups are topologically simple and act properly and cocompactly on a locally finite building that need be neither Euclidean nor hyperbolic, see [51]. In fact, all of these groups are abstractly simple by the main result of [40]. These groups also contain nonabelian discrete free groups, and so are nonamenable. Some variations of this construction are possible, see [52].

- Groups of tree automorphisms with Tits' property (P). A simplicity criterion for groups acting on trees has been given by Tits [62] and can be used to produce many examples. Some concrete examples have been constructed and studied in the groundbreaking work by Burger and Mozes [14]. The article [14] also contains a wealth of results on the structure of t.d.l.c. groups acting on trees, which have provided an important source of inspiration of the present work. A recent variation on the Burger-Mozes constructions is due to Smith [59]. Smith's construction takes as an input a pair of transitive permutation groups that are both generated by their stabilizers; one of them is required to be finite, and the other is to be compactly generated of countable degree with finite subdegrees. The output is a group in $\mathscr{S}$ acting on a biregular tree, with one class of vertices of finite degree and the other of infinite degree. By varying the infinite permutation group in the input, Smith obtains the first construction of uncountably many pairwise nonisomorphic group in $\mathscr{S}$.

- Other groups of tree automorphisms. Tits' property $(\mathrm{P})$ has been generalized by Banks et al. [4] and Le Boudec [37] to construct new examples of groups in $\mathscr{S}$; the groups in [37] act continuously but nonproperly on locally finite trees. Another simplicity criterion was developed by Möller and Vonk [41], although the present authors are not aware of any new examples arising from this criterion. 
- Groups of tree spheromorphisms. The prototypical examples in this family are the groups of tree spheromorphisms introduced by Neretin [46]: there is one such group for each regular locally finite tree $T$ of degree $d \geqslant 3$, with isomorphism type depending on $d$. Their simplicity is due to Kapoudjian [34]. Some variations have been constructed by Barnea et al. [5] and Caprace and De Medts [16].

- Groups acting on CAT(0) cube complexes. The aforementioned Tits' simplicity criterion for groups acting on trees can be generalized for groups acting on higher-dimensional CAT( 0$)$ cube complexes; examples have been obtained by Haglund and Paulin [31], Lazarovich [36] and Caprace [15]. A very general construction, incorporating many previous examples in this class and also generalizing the work of Burger-Mozes and Smith, was recently obtained by De Medts et al. [25].

Recall that we have divided the class $\mathscr{S}$ into five types, according to properties of the structure, centralizer and local decomposition lattices. We summarize here the division of the known examples among the five types.

It is known that simple algebraic groups over local fields do not admit any nontrivial compact locally normal subgroup, since each of their compact open subgroups is h.j.i. (see [54]). It is unknown whether Kac-Moody groups of compact hyperbolic type can be locally h.j.i. groups. However, in every other known example of groups in $\mathscr{S}$, the existence of a nontrivial compact locally normal subgroup has been observed. The type of the simple Kac-Moody groups is not known in general, although certainly they cannot be of atomic type, as they are all abstractly simple by [40].

Several families of groups in $\mathscr{S}$ acting on higher-dimensional CAT(0) cube complexes have been discovered. For all known groups in these families, there exist nondiscrete fixators of wings in the sense of [15] and their complements; this property in turn implies that the centralizer lattice is nontrivial, so these examples are either weakly or locally decomposable. For the examples in [15], a characterization is given of which of these examples are locally decomposable, from which it can be seen that not all examples are locally decomposable. In fact, as far as the present authors are aware, the nonlocally decomposable examples in [15] (and potentially some of their generalizations in [25]) are the only known examples of groups $G$ in $\mathscr{S}$ with $\mathcal{L D}(G)$ trivial but $\mathcal{L C}(G)$ nontrivial.

Once the examples of algebraic groups, Kac-Moody groups, and groups acting on higher-dimensional CAT(0) cube complexes are excluded, all remaining examples mentioned in this appendix are easily seen to be of locally decomposable type, that is, such that the local decomposition lattice is nontrivial. Many of these examples are obtained by using variations of Tits' 
simplicity criterion from [62]. That criterion, and its abstract version provided by Proposition I, is quite flexible and it is very likely that more examples of groups in $\mathscr{S}$ may be constructed by exploiting them. However, the 'wildness' which is inherent in the flexibility of this type of construction should be contrasted by the fact that the resulting groups in $\mathscr{S}$ will always be of weakly decomposable type, and thus fall into the subfamily for which the tools developed in the present paper are most powerful.

Remark Appendix A.1. Although most known examples are known to have nontrivial centralizer lattice, it is generally hard to determine if a given Boolean algebra in the centralizer lattice is actually the whole of $\mathcal{L C}(G)$ or $\mathcal{L D}(G)$, or merely a subalgebra. As far as the authors are aware, there are only two situations where $\mathcal{L C}(G)$ or $\mathcal{L D}(G)$ is nontrivial and has been completely determined:

(i) If a compact open subgroup of $G$ is a branch group, we can appeal to a result of Garrido [28] to conclude that, given any branch action of a compact open subgroup $U$ of $G$ on a rooted tree $T$, then $\mathcal{L D}(U)$, and hence $\mathcal{L} \mathcal{D}(G)$, is generated by the rigid stabilizers of $U$ acting on $T$, or equivalently the rigid stabilizers of $U$ acting on the boundary of $T$. In other words, in this situation the profinite spaces $\mathfrak{S}(\mathcal{L D}(G))$ and $\partial T$ are $G$-equivariantly isomorphic. This description covers Neretin's tree spheromorphism groups and the known examples of groups acting on locally finite trees with Tits' property $(\mathrm{P})$, and in these examples we see that $\mathcal{L D}(G)$ is $G$-equivariantly isomorphic to the algebra of clopen subsets of the space $\partial T$.

(ii) If $G \leqslant \operatorname{Aut}(T)$, where $T$ is a locally finite tree, such that $G$ is topologically simple and acts transitively on $\partial T$, then either $\mathcal{L C}(G)=\{0, \infty\}$ or $\mathfrak{S}(\mathcal{L C}(G))$ is $G$-equivariantly isomorphic to $\partial T$ : see Theorem Appendix B.2(i) below.

\section{Appendix B. Locally primitive groups of tree automorphisms}

A group $G$ of permutations of a set $X$ is quasiprimitive if every nontrivial normal subgroup of $G$ acts transitively on $X$.

A group $G$ of automorphisms of a locally finite tree $T$ is locally quasiprimitive if for every vertex $v$, the finite permutation group induced by the action of the stabilizer $G_{v}$ on the set $E(v)$ of edges emanating from $v$, is quasiprimitive.

Groups of tree automorphisms provide an important source of examples of groups in the class $\mathscr{S}$. The fundamental work of Burger and Mozes [14] has shown that the case where the action is locally quasiprimitive is especially rich 
and interesting. The goal of this appendix is to illustrate our results by specializing to that class. We first show that the only fixed points in the structure lattice are the trivial ones.

Proposition Appendix B.1. Let $T$ be a locally finite tree and $G \leqslant \operatorname{Aut}(T)$ be a closed edge-transitive subgroup which is locally quasiprimitive. If $G$ is topologically simple, then $\mathcal{L} \mathcal{N}(G)^{G}=\{0, \infty\}$.

Proof. Let $\alpha \in \mathcal{L N}(G)^{G} \backslash\{0\}$ be a nonzero fixed point of $G$ in the structure lattice. Let $e$ be an edge of $T$. By Lemma 2.4, the compact open subgroup $G_{e}$ contains a closed normal subgroup $L$ which is a representative of $\alpha$. Since $G$ acts properly and cocompactly on a tree, it is compactly presented by [24, Corollary 8.A.9]. Therefore, the last assertion of Proposition 5.17 ensures that the quotient $G_{e} / L$ is a topologically finitely generated, finite-by-abelian, profinite group. In particular it has a unique maximal finite subgroup. Let $K$ denote the preimage in $G_{e}$ of the maximal finite subgroup of $G_{e} / L$. Thus $K$ is a closed normal subgroup of $G_{e}$ representing $\alpha$, and $G_{e} / K$ is torsion-free. Moreover it follows from the construction that $K$ is the unique maximal closed subgroup of $G_{e}$ representing $\alpha$.

Let $v_{1}$ and $v_{2}$ be the two adjacent vertices that share the edge $e$. The compact subgroup $K$ cannot be normal both in $G_{v_{1}}$ and $G_{v_{2}}$, since it would then be normal in $\left\langle G_{v_{1}} \cup G_{v_{2}}\right\rangle=G$. Upon renaming $v_{1}$ and $v_{2}$, we assume henceforth that $K$ is not normal in $G_{v_{1}}$. Let $M_{1}$ be the (abstract) normal closure of $K$ in $G_{v_{1}}$. By [21, Corollary 7.13] we have $\left[M_{1}\right]=[K]=\alpha$. Note that $K$ is contained in $M_{1} \cap G_{e}$. Since $M_{1} \cap G_{e}$ is commensurate with $K$, and since $K$ is the unique maximal closed subgroup of $G_{e}$ representing $\alpha$, we infer that $M_{1} \cap G_{e}=K$.

Since $M_{1}$ is normal in $G_{v_{1}}$ while $K$ is not, it follows from the equality $M_{1} \cap G_{e}$ $=K$ that $M_{1}$ acts nontrivially on the set of edges $E\left(v_{1}\right)$ emanating from $v_{1}$. By hypothesis the $G_{v_{1}}$-action on $E\left(v_{1}\right)$ is quasiprimitive, hence $M_{1}$ is transitive on $E\left(v_{1}\right)$.

Now we distinguish two cases. Assume first that $K$ is not normal in $G_{v_{2}}$. We then denote the (abstract) normal closure of $K$ in $G_{v_{2}}$ by $M_{2}$. The same arguments as above show that $M_{2} \cap G_{e}=K$ and that $M_{2}$ is transitive on $E\left(v_{2}\right)$. It follows that $M_{1} \cap M_{2}=K$, and that $T$ is equivariantly isomorphic to the Bass-Serre tree of the amalgamated product $M_{1} *_{K} M_{2}$. In particular the group $D=\left\langle M_{1} \cup M_{2}\right\rangle$ acts properly and edge-transitively on $T$, and is thus closed in $G$. Since $D$ is edge-transitive, it acts transitively on the $G$-conjugacy class of $K$. Therefore, $D$ coincides with the normal closure of $K$ in $G$. Since $G$ is topologically simple, it follows that $D=G$. Thus $M_{1}=G_{v_{1}}$ and $M_{2}=G_{v_{2}}$, and both are open in $G$, so that $\alpha=\infty$. 
Consider next the case when $K$ is normal in $G_{v_{2}}$. We enumerate all the vertices at distance 1 from $v_{2}$ by $w_{1}=v_{1}, w_{2}, \ldots, w_{d}$, and for each $i$ we set $e_{i}=\left\{w_{i}\right.$, $\left.v_{2}\right\}$. Since $G_{v_{2}}$ is transitive on $E\left(v_{2}\right)=\left\{e_{1}, \ldots, e_{d}\right\}$ by hypothesis, it follows that $K$ is contained as a closed normal subgroup in $G_{e_{i}}$, and is the unique maximal closed subgroup of $G_{e_{i}}$ representing $\alpha$. For each $i=1, \ldots, d$, we denote by $M_{i}$ the normal closure of $K$ in $G_{w_{i}}$. We claim that $M_{i} \cap G_{e_{i}}=K$ and that $M_{i}$ is transitive on $E\left(w_{i}\right)$ for all $i$. Indeed, this has already been established for $i=1$, and the claim for $i>1$ follows from the transitivity of $G_{v_{2}}$ on $E\left(v_{2}\right)$. Noting that $M_{i} \cap M_{j}=K$ for all $i \neq j$ in $\{1, \ldots, d\}$, we see that $T$ coincides with the Bass-Serre tree of the amalgamated product $M_{1} *_{K} M_{2} *_{K} \cdots *_{K} M_{d}$ (viewed as the fundamental group of a finite tree of groups, the finite tree in question being isomorphic to the $\operatorname{star} S=e_{1} \cup \cdots \cup e_{d}$ ). It follows that the group $D=$ $\left\langle M_{1} \cup \cdots \cup M_{d}\right\rangle$ acts properly on $T$ with the star $S$ as a fundamental domain. In particular $D$ is closed in $G$, and $D$ is transitive on the $G$-conjugacy class of $K$. As in the previous case, this implies that $D$ is normal, hence open in $G$, so that $K$ is of countable index in $G$. Therefore, $K$ is open and $\alpha=\infty$.

We emphasize that, under the hypothesis that $G$ is locally quasiprimitive, it follows from [14, Proposition 1.2.1] that $G$ is topologically simple as soon as it has no nontrivial discrete normal subgroup, and no proper open normal subgroup of finite index.

We next turn to the case where $G$ is doubly transitive on the set of ends $\partial T$. This implies that $G$ is locally 2 -transitive, hence locally primitive. In fact, if $G \leqslant$ $\operatorname{Aut}(T)$ is closed, noncompact and transitive on $\partial T$, then it is 2-transitive on $\partial T$ and locally 2-transitive: see [14, Lemma. 3.1.1].

THEOREM Appendix B.2. Let $T$ be an infinite locally finite tree and $G \leqslant \operatorname{Aut}(T)$ be a closed subgroup which is topologically simple and acts transitively on the set of ends $\partial T$. Then:

(i) The Stone space $\mathfrak{S}(\mathcal{L C}(G))$ is either trivial, or equivariantly homeomorphic to $\partial T$. In particular $\mathcal{L C}(G)$ is either trivial or countable.

(ii) $\mathcal{L C}(G) \neq\{0, \infty\}$ if and only if the pointwise stabilizer of some half-tree is nontrivial.

(iii) If $\mathcal{L C}(G) \neq\{0, \infty\}$, then $G$ is abstractly simple.

Proof. (i) Since $T$ is infinite and locally finite, the set of ends $\partial T$ is nonempty. Let $\xi \in \partial T$ and $P=G_{\xi}$ be the stabilizer of $\xi$. Then the set of elliptic elements of $P$ is an open normal subgroup $P_{0}$ of $P$, which is locally elliptic, and the quotient $P / P_{0}$ is cyclic. In particular $P$ is amenable. The canonical equivariant bijection 
$G / P \rightarrow \partial T$ is continuous, and must therefore be a homeomorphism by [3, Theorem 8]. It follows that $G / P$ is compact. Moreover $P$ is a maximal subgroup of $G$ since the $G$-action on $G / P$ is doubly transitive (by [14, Lemma 3.1.1]), hence primitive. Therefore, by Proposition 6.25, every compact $G$-space which is minimal and strongly proximal either is isomorphic to $G / P \cong \partial T$, or is a singleton. In particular, this applies to the Stone space $\mathfrak{S}(\mathcal{L C}(G))$ by Theorem J. This proves (i).

(ii) Given any pair of half-trees $H_{1}, H_{2}$ in $T$, there is an element $g \in G$ mapping $H_{1}$ properly inside $H_{2}$. The 'if' part follows easily from that observation. Assume conversely that $\mathcal{L C}(G)$ is nontrivial. Then $\mathfrak{S}(\mathcal{L C}(G))$ can be identified with $\partial T$ by (i). Since the $G$-action on $\mathfrak{S}(\mathcal{L C}(G))$ is locally weakly decomposable by Theorem $\mathrm{J}$, it follows that the pointwise stabilizer in $G$ of any nonempty proper clopen subset of $\partial T$ is nontrivial. The conclusion follows, since the pointwise stabilizer of a nonempty proper clopen subset of $\partial T$ fixes pointwise a half-tree of $T$.

(iii) Upon replacing $T$ by a minimal $G$-invariant subtree, and discarding the vertices of degree 2, we may assume that the $G$-action on $T$ is edge-transitive. By [14, Lemma 3.1.1], the $G$-action on $\partial T$ is 2-transitive, and the $G$-action on $T$ is locally 2-transitive, hence locally quasiprimitive. The conclusion then follows from Proposition Appendix B.1 and Theorem P.

In particular, in this case we can solve the problem posed by Remark 6.26.

Corollary Appendix B.3. Let $T$ be an infinite locally finite tree and $G \leqslant$ $\operatorname{Aut}(T)$ be a closed subgroup that is topologically simple and acts transitively on the set of ends $\partial T$. Then every orbit of $G$ on $\mathcal{L C}(G) \backslash\{0, \infty\}$ is minorizing.

Proof. We may assume that $\mathcal{L C}(G)$ is nontrivial. Then by Theorem Appendix B.2(i), the Stone space $\mathfrak{S}(\mathcal{L C}(G))$ is $G$-equivariantly homeomorphic to $\partial T$; in other words, we may identify $\mathcal{L C}(G)$ with the Boolean algebra $\mathcal{A}$ of clopen subsets of $\partial T$. Given a directed edge $e$ in $T$, let $S_{e}$ be the half-tree of vertices closer to $o(e)$ than to $t(e)$. Then the set $\alpha_{e}$ of ends of $S_{e}$ is an element of $\mathcal{A}$; moreover, it is easily seen that the set

$$
\mathcal{H}:=\left\{\alpha_{e} \mid e \in E T\right\}
$$

is minorizing in $\mathcal{A}$. Now [14, Lemma. 3.1.1] implies that $G$ is transitive on undirected edges of $T$; since $G$ is topologically simple, $G$ preserves the natural bipartition of the vertices. Hence $G$ has exactly two orbits of the directed edges of $T$, so that $e$ and $e^{\prime}$ lie in the same orbit if and only if $o(e)$ and $o\left(e^{\prime}\right)$ lie in the same part of the bipartition. We see that for any two edges $e, e^{\prime}$, there exists $g \in G$ 
such that the undirected edges of $g e$ and $e^{\prime}$ are distinct, and either $o\left(e^{\prime}\right)=t(g e)$ or there is an edge $e^{\prime \prime}$ from $o\left(e^{\prime}\right)$ to $t(g e)$. In either case we see that $S_{g e} \subset S_{e^{\prime}}$ and hence $g \alpha_{e}<\alpha_{e^{\prime}}$. Thus $G \alpha_{e}$ is minorizing in $\mathcal{H}$, and hence in $\mathcal{A}$, for all $e \in E T$. In particular, since $\left(\alpha_{e}\right)^{\perp}=\alpha_{\bar{e}}$, we obtain an element $\alpha \in \mathcal{L C}(G)$ such that both $\alpha$ and $\alpha^{\perp}$ are minorizing under the $G$-action. Given $\beta, \gamma \in \mathcal{L C}(G) \backslash\{0, \infty\}$, there exist $g, h \in G$ such that $g^{-1} \alpha^{\perp}<\beta^{\perp}$ and $h \alpha<\gamma$; these two inequalities imply that $h g \beta<\gamma$. Hence every orbit of $G$ on $\mathcal{L C}(G) \backslash\{0, \infty\}$ is minorizing.

\section{Index}

[A]-regular radical, 39

abstractly linear, 14

abstractly simple, 2, 13

action

locally weakly decomposable, 11 minimal, 11 strongly proximal, 11

algebraic group, 77

amenable, 12

$\mathcal{B}(G), 14$

C-stable, 16

Cayley-Abels graph, 28

centralizer lattice, 5, 16

commensurate, 14

compactly generated, 2

compactly presented, 45

compressible, 9

compressible to a point, 59

$\operatorname{con}(g), 12,61$

contraction group, 12, 61

$\eta(G), 30$

extremely proximal, 74

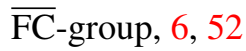

filter, 42

free subsemigroup, 12

$G_{(\mu)}, 45$

Hecke pair, 19

hereditarily just-infinite, 7

Higman's simplicity criterion, 10

h.j.i., 7

just-infinite relatively, 21

just-noncompact, 22

Kac-Moody group, 78

large, 24

$\mathcal{L C}(G), 5,16$

$\mathcal{L D}(G), 5,16$

linear, 14

$\mathcal{L N}(G), 5$

local decomposition lattice, 5, 16

local prime content, 30

local size, 24

localization, 45

localized topology, 45

locally C-stable, 16

locally elliptic subgroup, 52 
locally equivalent, 14

locally normal subgroup, 4, 14 trivial, 4

locally sized, 24

locally weakly decomposable action, 11

Margulis-Zimmer conjecture, 23

maximal, 17

meagre, 24

microsupported, 9

minimal, 17

minimal action, 11, 71

minorizing, 59

degree, 59

set, 59

$\operatorname{Mon}(G), 20$

monolith, 20

monolithic, 20

$O_{\infty}(G), 30$

$O^{\infty}(G), 30$

$O_{\pi}(G), 30$

$O^{\pi}(G), 30$

p-adic Lie group, 4, 77

partition, 57

refinement of a, 57

$\pi$-core, 30

$\pi$-residual, 30

Polish group, 12

profinite space, 10, 55

prosoluble core, 30

prosoluble residual, 30 proximal action, 71

$\mathrm{QC}_{H}(K), 15$

quasicentralizer, 15

quasicentre, 16

quasiprimitive, 80

locally quasiprimitive, 80

quasiproduct, 21

$\mathrm{QZ}(G), 16$

$\mathrm{R}_{[\mathrm{A}]}(G), 39$

refinement, 57

relatively simple, 21

rigid stabilizer, 56

$\operatorname{rist}_{G}(v), 56$

$\mathscr{S}, 2$

(S0), 5

(S1), 5

(S2), 5

simple

relatively, 21

topologically, 2

$K$-sized, 24

skewering element, 61

smooth action, 56

Stone space, 10, 55

strongly proximal action, 11,71

structure lattice, 5, 14

fixed points in, 6

t.d.l.c.s.c., 15

t.d.l.c., 2

Tits core, 61

topologically simple, 2

tree, 78 
unrefinable, 26

upper subset, 42 weakly decomposable, 11

locally, 11

\section{References}

[1] H. Abels, 'Kompakt definierbare topologische Gruppen', Math. Ann. 197 (1972), 221-233.

[2] H. Abels, 'Specker-Kompaktifizierungen von lokal kompakten topologischen Gruppen', Math. Z. 135 (1974), 325-361.

[3] R. Arens, 'Topologies for homeomorphism groups', Amer. J. Math. 68 (1946), 593-610.

[4] C. Banks, M. Elder and G. A. Willis, 'Simple groups of automorphisms of trees determined by their actions on finite subtrees', J. Group Theory 18(2) (2015), 235-261.

[5] Y. Barnea, M. Ershov and T. Weigel, 'Abstract commensurators of profinite groups', Trans. Amer. Math. Soc. 363(10) (2011), 5381-5417.

[6] U. Baumgartner, J. Ramagge and B. Rémy, 'Contraction groups in complete Kac-Moody groups', Groups Geom. Dyn. 2(3) (2008), 337-352.

[7] U. Baumgartner and G. A. Willis, 'Contraction groups and scales of automorphisms of totally disconnected locally compact groups', Israel J. Math. 142 (2004), 221-248.

[8] V. V. Belyaev, 'Locally finite groups with a finite nonseparable subgroup (Russian) Sibirsk', Mat. Ž. 34(2) (1993), 23-41. 226, 233. Translation in Siberian Math. J. 34 (1993), no. 2, 218-232 (1994).

[9] R. Bieri and R. Strebel, On Groups of PL-Homeomorphisms of the Real Line, Mathematical Surveys and Monographs, 215 (American Mathematical Society, Providence, RI, 2016).

[10] A. Borel and J. Tits, 'Homomorphismes 'abstraits' de groupes algébriques simples', Ann. of Math. 97 (1973), 499-571.

[11] N. Bourbaki, 'Groupes et algèbres de Lie', in Chapitre II: Algèbres de Lie libres. Chapitre III: Groupes de Lie, Actualités Scientifiques et Industrielles, 1349 (Hermann, Paris, 1972), 320.

[12] A. Bouziad and J.-P. Troallic, 'Some remarks about strong proximality of compact flows', Colloq. Math. 115(2) (2009), 159-170.

[13] F. Bruhat and J. Tits, 'Groupes réductifs sur un corps local', Publ. Math. Inst. Hautes Études Sci. 41 (1972), 5-251.

[14] M. Burger and Sh. Mozes, 'Groups acting on trees: from local to global structure', Publ. Math. Inst. Hautes Études Sci. 92 (2000), 113-150.

[15] P.-E. Caprace, 'Automorphism groups of right-angled buildings: simplicity and local splittings', Fund. Math. 224 (2014), 17-51.

[16] P.-E. Caprace and T. De Medts, 'Simple locally compact groups acting on trees and their germs of automorphisms', Transform. Groups 16(2) (2011), 375-411.

[17] P.-E. Caprace and T. De Medts, 'Trees, contraction groups, and Moufang sets', Duke Math. J. 162(13) (2013), 2413-2449.

[18] P.-E. Caprace and N. Monod, 'Decomposing locally compact groups into simple pieces', Math. Proc. Cambridge Philos. Soc. 150(1) (2011), 97-128.

[19] P.-E. Caprace, C. D. Reid and G. A. Willis, 'Locally normal subgroups of simple locally compact groups', C. R. Acad. Sci. Paris Ser. I 351(17-18) (2013), 657-661.

[20] P.-E. Caprace, C. D. Reid and G. A. Willis, 'Limits of contraction groups and the Tits core', J. Lie Theory 24(4) (2014), 957-967. 
[21] P.-E. Caprace, C. D. Reid and G. A. Willis, 'Locally normal subgroups of totally disconnected groups; Part I: general theory', Forum of Mathematics, Sigma 5 (2017), doi:10.1017/fms.2017.9.

[22] P.-E. Caprace and T. Stulemeijer, 'Totally disconnected locally compact groups with a linear open subgroup', Int. Math. Res. Not. IMRN 24 (2015), 13800-13829.

[23] R. Cluckers, Y. Cornulier, N. Louvet, R. Tessera and A. Valette, 'The Howe-Moore property for real and p-adic groups', Math. Scand. 109(2) (2011), 201-224.

[24] Y. Cornulier and P. de la Harpe, Metric Geometry of Locally Compact Groups, EMS Tracts in Mathematics, 25 (European Math. Soc., Zurich, 2016).

[25] T. De Medts, A. C. Silva and K. Struyve, 'Universal groups for right-angled buildings', Preprint, 2016, arXiv:1603.04754.

[26] D. B. A. Epstein, 'The simplicity of certain groups of homeomorphisms', Compos. Math. 22 (1970), 165-173.

[27] H. Furstenberg, 'Boundary theory and stochastic processes on homogeneous spaces', in Harmonic Analysis on Homogeneous Spaces, Proc. Sympos. Pure Math., XXVI (Williams Coll., Williamstown, Mass., 1972) (Amer. Math. Soc., Providence, RI, 1973), 193-229.

[28] A. Garrido, 'On the congruence subgroup problem for branch groups', Israel J. Math. 216(1) (2016), 1-13.

[29] S. Glasner, 'Topological dynamics and group theory', Trans. Amer. Math. Soc. 187 (1974), 327-334.

[30] K. W. Gruenberg, 'Residual properties of infinite soluble groups', Proc. Lond. Math. Soc. 7(3) (1957), 29-62.

[31] F. Haglund and F. Paulin, 'Simplicité de groupes d'automorphismes d'espaces à courbure négative', in The Epstein Birthday Schrift, Geom. Topol. Monogr., 1 (Geom. Topol. Publ., Coventry, 1998), 181-248. (electronic).

[32] K. Juschenko and N. Monod, 'Cantor systems, piecewise translations and simple amenable groups', Ann. of Math. (2) 178(2) (2013), 775-787.

[33] S. Kakutani and K. Kodaira, 'Über das Haarsche Mass in der lokal bikompakten Gruppe', Proc. Imp. Acad. Tokyo 20 (1944), 444-450.

[34] C. Kapoudjian, 'Simplicity of Neretin's group of spheromorphisms', Ann. Inst. Fourier (Grenoble) 49(4) (1999), 1225-1240.

[35] L. Kramer, 'The topology of a semisimple Lie group is essentially unique', Adv. Math. 228(5) (2011), 2623-2633.

[36] N. Lazarovich, 'On regular CAT(0) cube complexes', Preprint, 2014, arXiv:1411.0178.

[37] A. Le Boudec, 'Adrien Groups acting on trees with almost prescribed local action', Comment. Math. Helv. 91(2) (2016), 253-293.

[38] A. Malcev, 'On isomorphic matrix representations of infinite groups', Mat. Sb. 8(50) (1940), 405-422 (Russian).

[39] G. Margulis, Discrete Subgroups of Semi-Simple Lie Groups, Ergebnisse der Mathematik und ihrer Grenzgebiete (3), 17 (Springer, Berlin, 1991).

[40] T. Marquis, 'Abstract simplicity of locally compact Kac-Moody groups', Compos. Math. 150(4) (2014), 713-728.

[41] R. Möller and J. Vonk, 'Normal subgroups of groups acting on trees and automorphism groups of graphs', J. Group Theory 15(6) (2012), 831-850.

[42] N. Monod, Continuous Bounded Cohomology of Locally Compact Groups, Lecture Notes in Mathematics, 1758 (Springer, Berlin, 2001).

[43] D. Montgomery and L. Zippin, Topological Transformation Groups, (Interscience Publishers, New York-London, 1955). 
[44] V. Nekrashevych, 'Finitely presented groups associated with expanding maps'. Preprint, 2013, arXiv:1312.5654.

[45] V. Nekrashevych, 'Palindromic subshifts and simple periodic groups of intermediate growth', Preprint, 2016, arXiv:1601.01033.

[46] Yu. A. Neretin, 'On combinatorial analogs of the group of diffeomorphisms of the circle', Izv. Math. 41(2) (1993), 337-349 (Russian).

[47] N. Nikolov, 'Algebraic properties of profinite groups', Preprint, 2011, arXiv:1108.5130.

[48] N. Nikolov and D. Segal, 'Generators and commutators in finite groups; abstract quotients of compact groups', Invent. Math. 190(3) (2012), 513-602.

[49] C. D. Reid and P. Wesolek, 'Homomorphisms into totally disconnected, locally compact groups with dense image', Preprint, 2015, arXiv:1509.00156v1.

[50] C. D. Reid and P. Wesolek, 'The essentially chief series of a compactly generated locally compact group', Preprint, 2015, arXiv:1509.06593v4.

[51] B. Rémy, 'Topological simplicity, commensurator superrigidity and non-linearities of KacMoody groups', Geom. Funct. Anal. 14(4) (2004), 810-852. With an appendix by P. Bonvin.

[52] B. Rémy and M. Ronan, 'Topological groups of Kac-Moody type, right-angled twinnings and their lattices', Comment. Math. Helv. 81(1) (2006), 191-219.

[53] L. Ribes and P. Zalesskii, Profinite Groups, 2nd edn, Ergebnisse der Mathematik und ihrer Grenzgebiete. 3. Folge A Series of Modern Surveys in Mathematics [Results in Mathematics and Related Areas. 3rd Series. A Series of Modern Surveys in Mathematics], 40 (Springer, Berlin, 2010).

[54] C. Riehm, 'The congruence subgroup problem over local fields', Amer. J. Math. 92 (1970), 771-778.

[55] M. Rosenlicht, 'On a result of Baer', Proc. Amer. Math. Soc. 13 (1962), 99-101.

[56] M. Rubin, 'On the reconstruction of topological spaces from their groups of homeomorphisms', Trans. Amer. Math. Soc. 312(2) (1989), 487-538.

[57] J. Schur, 'Über die Darstellung der endlichen Gruppen durch gebrochen lineare Substitutionen', J. Reine Angew. Math. 127 (1904), 20-50.

[58] Y. Shalom and G. A. Willis, 'Commensurated subgroups of arithmetic groups, totally disconnected groups and adelic rigidity', Geom. Funct. Anal. 23(5) (2013), 1631-1683.

[59] S. M. Smith, 'A product for permutation groups and topological groups', Preprint, 2015, arXiv:1407.5697v2.

[60] S. Thomas and B. Velickovic, 'On the complexity of the isomorphism relation for finitely generated groups', J. Algebra 217(1) (1999), 352-373.

[61] J. Tits, 'Algebraic and abstract simple groups', Ann. of Math. 80 (1964), 313-329.

[62] J. Tits, 'Sur le groupe des automorphismes d'un arbre', in Essays on Topology and Related Topics (Mémoires dédiés à Georges de Rham) (Springer, New York, 1970), 188-211.

[63] J. Tits, 'Reductive groups over local fields', in Automorphic Forms, Representations and L-Functions (Proc. Sympos. Pure Math., Oregon State Univ., Corvallis, Ore., 1977), Part 1, Proc. Sympos. Pure Math., XXXIII (Amer. Math. Soc., Providence, RI, 1979), 29-69.

[64] J. Tits, 'Uniqueness and presentation of Kac-Moody groups over fields', J. Algebra 105(2) (1987), 542-573.

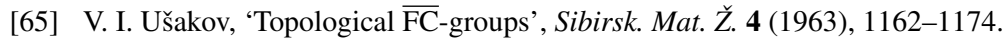

[66] S. P. Wang, 'Compactness properties of topological groups', Trans. Amer. Math. Soc. 154 (1971), 301-314.

[67] P. Wesolek, 'Elementary totally disconnected locally compact groups', Proc. Lond. Math. Soc. 110(6) (2015), 1387-1434. 
[68] P. Wesolek, 'Commensurated subgroups in finitely generated branch groups', J. Group Theory 20(2) (2017), 385-392.

[69] G. A. Willis, 'The number of prime factors of the scale function on a compactly generated group is finite', Bull. Lond. Math. Soc. 33(2) (2001), 168-174.

[70] G. A. Willis, 'Compact open subgroups in simple totally disconnected groups', J. Algebra 312(1) (2007), 405-417.

[71] G. A. Willis, 'The nub of an automorphism of a totally disconnected, locally compact group', Ergodic Theory Dynam. Systems 34(4) (2014), 1365-1394. 